\title{
Numerical Examination of Flow Field Characteristics and \\ Fabri Choking of 2D Supersonic Ejectors
}

\author{
A Thesis \\ Presented to the Faculty of \\ California Polytechnic State University \\ San Luis Obispo \\ In Partial Fulfillment of the Requirements \\ For the Degree of Master of Science \\ In Aerospace Engineering
}

by

Brett G. Morham

June 2010 
(C) 2010

Brett G Morham

ALL RIGHTS RESERVED 


\section{COMMITTEE MEMBERSHIP}

Title: $\quad$ Numerical Examination of Flow Field Characteristics and Fabri Choking of 2D Supersonic Ejectors

Author: $\quad$ Brett G Morham

Date Submitted: June 2010

Advisor and Committee Chair:

Dr. Dianne DeTurris

Faculty Committee Member:

Dr. David Marshall

Faculty Committee Member:

Dr. William Durgin

Industry Committee Member: Ryan Gist 


\section{ABSTRACT \\ Numerical Examination of Flow Field Characteristics and Fabri Choking \\ of 2D Supersonic Ejectors \\ Brett Morham}

An automated computer simulation of the two-dimensional planar Cal Poly Supersonic Ejector test rig is developed. The purpose of the simulation is to identify the operating conditions which produce the saturated, Fabri choke and Fabri block aerodynamic flow patterns. The effect of primary to secondary stagnation pressure ratio on the efficiency of the ejector operation is measured using the entrainment ratio which is the secondary to primary mass flow ratio.

The primary flow of the ejector is supersonic and the secondary (entrained) stream enters the ejector at various velocities at or below Mach 1. The primary and secondary streams are both composed of air. The primary plume boundary and properties are solved using the Method of Characteristics. The properties within the secondary stream are found using isentropic relations along with stagnation conditions and the shape of the primary plume. The solutions of the primary and secondary streams iterate on a pressure distribution of the secondary stream until a converged solution is attained. Viscous forces and thermo-chemical reactions are not considered.

For the given geometry the saturated flow pattern is found to occur below stagnation pressure ratios of 74. The secondary flow of the ejector becomes blocked by the primary plume above pressure ratios of 230. The Fabri choke case exists between pressure ratios of 74 and 230, achieving optimal operation at the transition from saturated to Fabri choked flow, near the pressure ratio of 74. The case of optimal expansion yields an entrainment ratio of 0.17. The entrainment ratio results of the Cal Poly Supersonic Ejector simulation have an average error of $3.67 \%$ relative to experimental data. The accuracy of this inviscid simulation suggests ejector operation in this regime is governed by pressure gradient rather than viscous effects. 


\section{Acknowledgments}

I must thank my family, my friends, and my thesis committee. My parents Eric and Olga Morham and my brothers Kyle and Sean have provided endless encouragement, motivation and generosity. Without my family, none of this would have been possible. My friends, especially Rae Boghossian, have offered unlimited patience and optimism. Without this balance, I would never have been able to complete this project.

I must acknowledge Dr. DeTurris for inspiring me and opening my eyes to the world of hypersonics and this project. Dr. DeTurris has kept me on track and motivated, selflessly allocating time for support and advising. Ryan Gist has acted as a mentor before and throughout this project. Ryan and his colleagues at Rocketdyne, especially Bill Follett, have provided invaluable perspective. Thank you to Joey Sanchez and Martin Popish for having faith in this project and coming on board, keeping the project

alive. Also, thank you Paul Riley for providing a working MOC nozzle code which acted as a backbone for the simulation.

I would like to thank my thesis committee as a whole; Dr. DeTurris, Ryan Gist, Dr. Durgin and Dr. Marshall. Thank you for taking the time and providing the insight to make this thesis what it now is.

Finally, I dedicate this work to Mark Waters. He was a true mentor to the world of propulsion. The influence of his passion and dedication to the field is immeasurable. 


\section{Table of Contents}

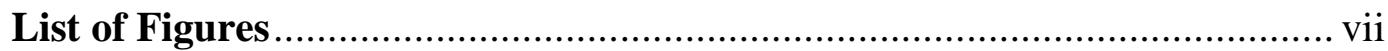

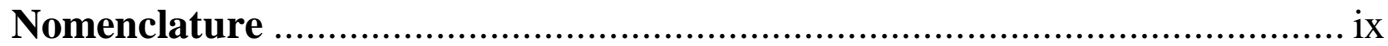

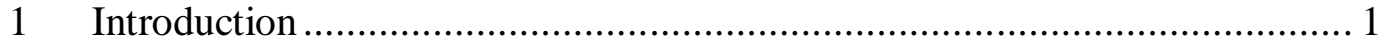

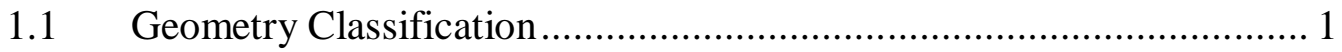

$1.2 \quad$ Flow Composition Classification ...................................................... 3

1.3 Flow Velocity Classification ……………........................................ 4

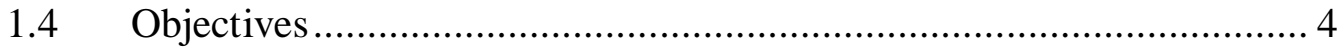

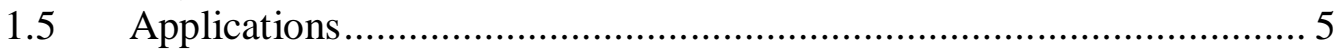

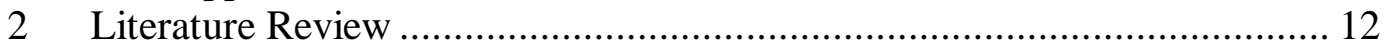

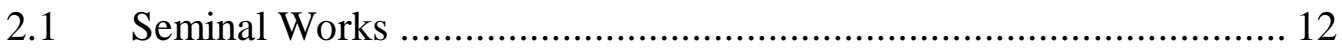

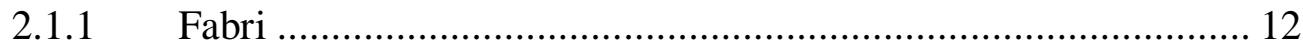

2.1.2 Emanuel ....................................................................... 15

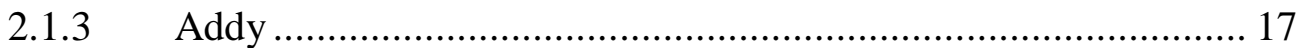

2.2 Supplemental Analysis Approaches ............................................... 19

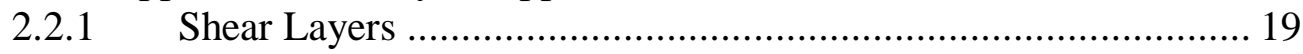

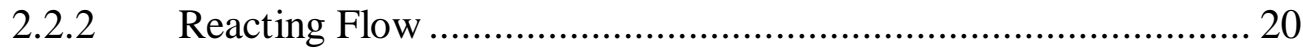

2.2.3 Lobed Ejectors ................................................................... 20

2.2.4 Computational Fluid Dynamics ................................................. 20

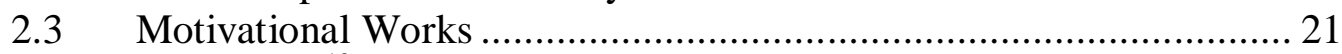

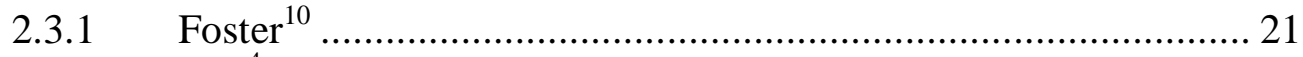

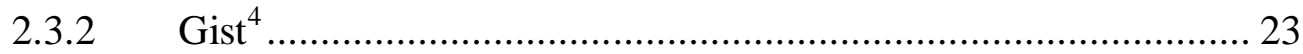

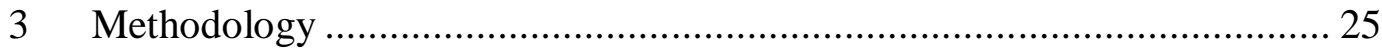

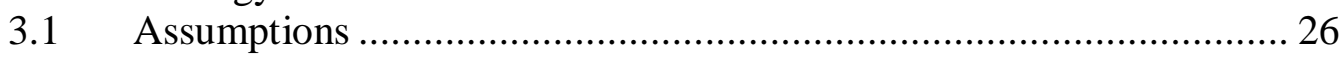

3.2 Primary Plume Calculation Method.................................................... 26

3.2.1 Method of Characteristics Interior Point Calculation ................... 28

3.2.2 Method of Characteristics Direct Wall Point Calculation............. 32

3.2.3 Method of Characteristics Free Pressure Boundary Point

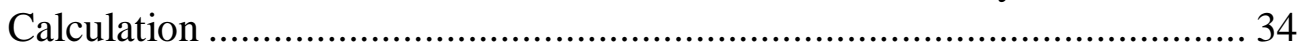

3.3 Secondary Stream Calculation Method.............................................. 37

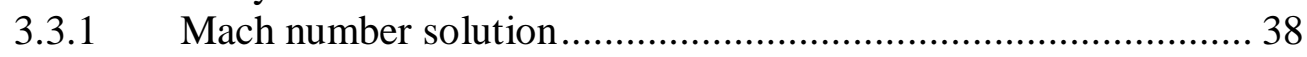

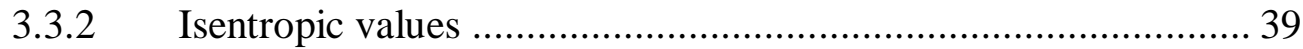

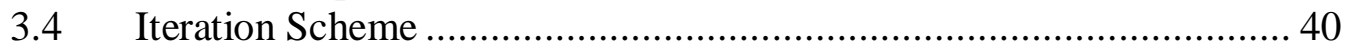

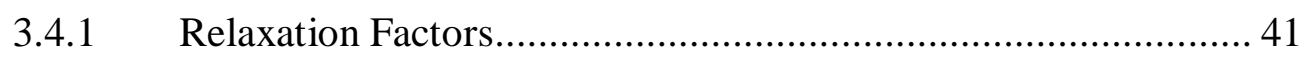

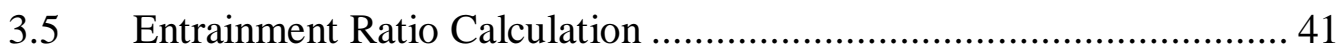

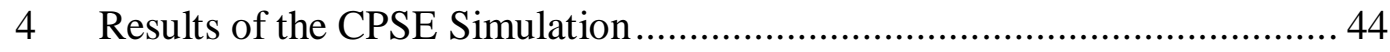

4.1 Saturated Supersonic Flow Pattern ................................................ 45

4.2 Fabri Choke Supersonic Flow Pattern ............................................. 49

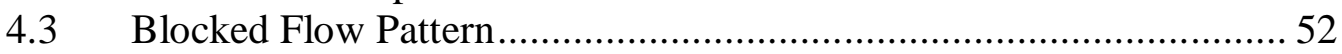

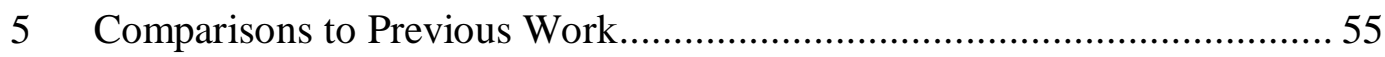

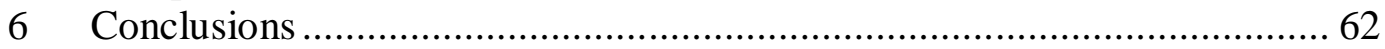

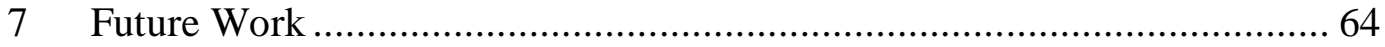

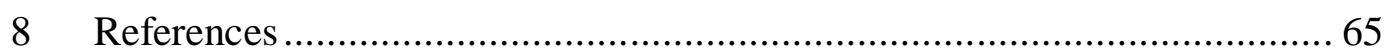

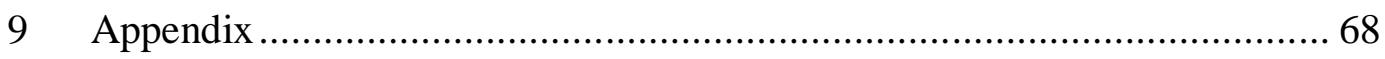




\section{List of Figures}

Figure 1-1 Top and front view of an axisymmetric ejector .......................................... 1

Figure 1-2 Top and front view of a two-dimensional planar ejector .............................2

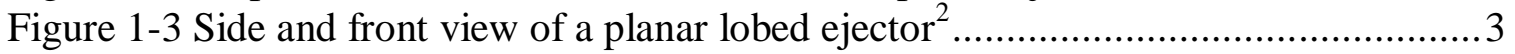

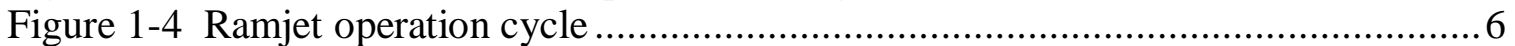

Figure 1-5: Effects of Mach number of efficiency of propulsion systems ${ }^{4}$....................... 7

Figure 1-6 X-43 Hypersonic test vehicle 3-view ${ }^{5}$.................................................... 8

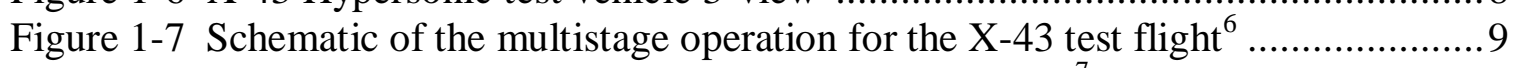

Figure 1-8 SR-71 turbine based combined cycle vehicle 3 -view ${ }^{7}$....................................

Figure 1-9 Turbine based combined cycle engine integration ${ }^{9}$...................................... 10

Figure 1-10 Concept vehicle incorporating a rocket based combined cycle

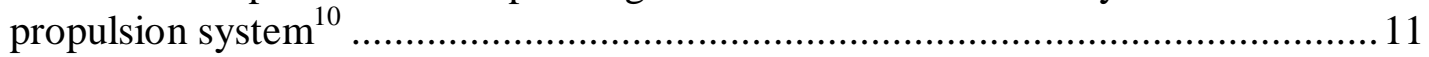

Figure 2-1 Secondary flow achieves critical Mach number during the Fabri choke

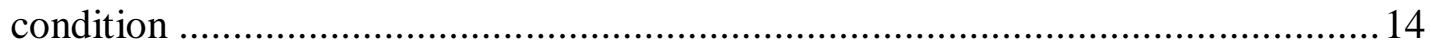

Figure 2-2 The secondary flow entering the mixing chamber is sonic during the

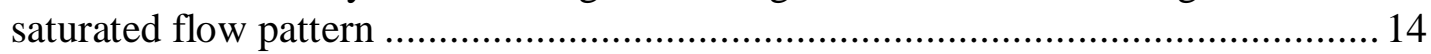

Figure 2-3 The primary flow becomes subsonic during the subsonic flow pattern ......... 15

Figure 2-4 The Cal Poly Supersonic Ejector ……………........................................22

Figure 2-5 The variation between ideal and actual primary plume expansion ${ }^{4}$................25

Figure 2-6 Growth of primary plume from ideal to actual size due to empirical

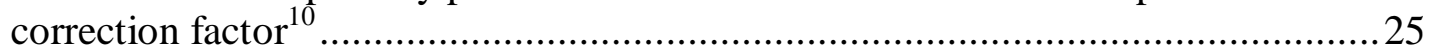

Figure 3-1 Definition of primary plume using Method of Characteristics......................22

Figure 3-2 Method of Characteristics unit process for an interior point ${ }^{25} \ldots \ldots \ldots \ldots \ldots \ldots \ldots \ldots . .28$

Figure 3-3 Definition of angles used in Method of Characteristics ................................30

Figure 3-4 Method of Characteristics unit process for a symmetry point ${ }^{25} \ldots \ldots \ldots \ldots \ldots \ldots \ldots . . . .31$

Figure 3-5 Method of Characteristics unit process for a wall point ${ }^{25}$............................. 33

Figure 3-6 Method of Characteristics unit process for free pressure boundary

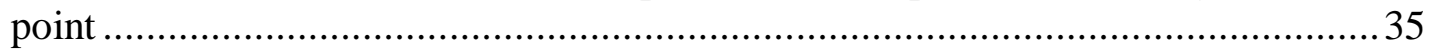

Figure 3-7 Components of the velocity vector along the free pressure boundary

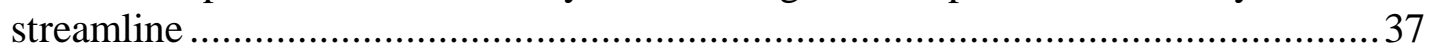

Figure 3-8 The Mach number of secondary flow using isentropic area relations............ 38

Figure 4-1 Dimensioned top view of the CPSE test apparatus ${ }^{4}$..................................... 45

Figure 4-2 CPSE simulation of the Mach number distribution of a saturated flow

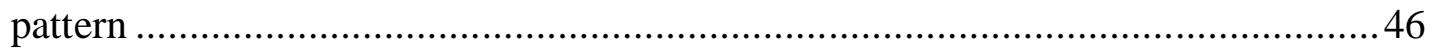

Figure 4-3 CPSE simulation of the static pressure distribution of a saturated flow

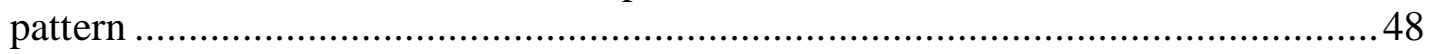

Figure 4-4 CPSE simulation of the Mach number distribution of a Fabri choke

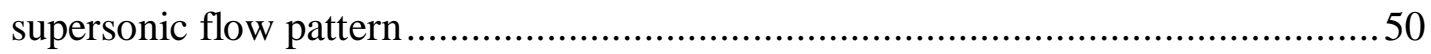

Figure 4-5 CPSE simulation of the static pressure distribution of a Fabri choke

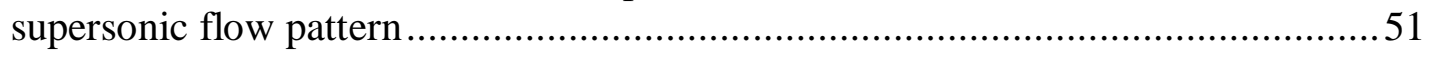

Figure 4-6 CPSE simulation of the Mach number distribution of a blocked flow

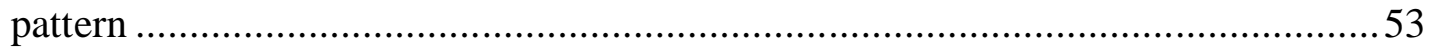

Figure 4-7 CPSE simulation of the static pressure distribution of a blocked flow

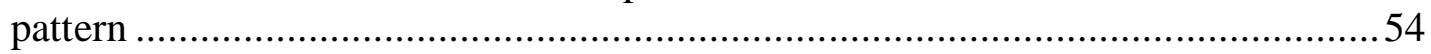

Figure 5-1 Nitrogen condensation plume in the mixing chamber at a pressure ratio of $95^{4}$ 
Figure 5-2 Methane and Oxygen thruster fired at a pressure ratio of $20^{10} \ldots \ldots \ldots \ldots \ldots \ldots . . . . . .56$

Figure 5-3 Video frame and superimposed shock structure from cold flow tests ${ }^{4} \ldots \ldots \ldots . .57$

Figure 5-4 Mach Number and Streamlines from CFD Model $^{30}$..................................58

Figure 5-5 Entrainment ratio calculations using empirical correction factor for plume size 4

Figure 5-6 Comparison of CPSE computer code to experimental data and

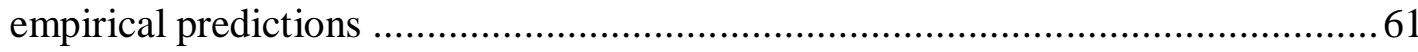




\section{Nomenclature}

\begin{tabular}{|c|c|c|}
\hline $\mathrm{a}$ & Speed of Sound & {$[\mathrm{ft} / \mathrm{s}]$} \\
\hline A & Area & {$\left[\mathrm{ft}^{2}\right],\left[\mathrm{in}^{2}\right]$} \\
\hline $\mathrm{C}$ & Characteristic Curve (Mach Line) & - \\
\hline $\mathrm{h}$ & Ejector height & {$[\mathrm{ft}],[\mathrm{in}]$} \\
\hline $\mathrm{I}_{\mathrm{sp}}$ & Specific Impulse & $(\mathrm{s})$ \\
\hline $\mathrm{K}$ & Relaxation Factor & - \\
\hline $\mathrm{K}_{\text {expand }}$ & Plume Expansion Correction Factor & - \\
\hline $\mathrm{M}$ & Mach Number & - \\
\hline$\dot{m}$ & Mass Flow Rate & {$\left[1 \mathrm{~b}_{\mathrm{m}} / \mathrm{s}\right]$} \\
\hline $\mathrm{P}$ & Stagnation Pressure & {$\left[1 \mathrm{lb}_{\mathrm{f}} / \mathrm{ft}^{2}\right]$} \\
\hline $\mathrm{p}$ & Static Pressure & {$\left[1 b_{f} / \mathrm{ft}^{2}\right]$} \\
\hline $\mathrm{Q}, \mathrm{R}, \mathrm{T}$ & Coefficients in Finite Difference Equations & - \\
\hline $\mathrm{R}$ & Universal Gas Constant & {$\left[\mathrm{lb}_{\mathrm{f}}-\mathrm{ft} / \mathrm{lb} \mathrm{b}_{\mathrm{m}}{ }^{\circ} \mathrm{R}\right]$} \\
\hline $\mathrm{T}$ & Temperature & {$\left[{ }^{\circ} \mathrm{F},{ }^{\circ} \mathrm{R}\right]$} \\
\hline$t_{b}$ & Primary Nozzle Base Thickness & {$[\mathrm{ft}],[\mathrm{in}]$} \\
\hline$u$ & Transverse Velocity Component & {$[\mathrm{ft} / \mathrm{s}]$} \\
\hline$v$ & Streamwise Velocity Component & {$[\mathrm{ft} / \mathrm{s}]$} \\
\hline $\mathrm{V}$ & Total Velocity & {$[\mathrm{ft} / \mathrm{s}]$} \\
\hline $\mathrm{x}$ & Streamwise Position From Primary Throat & {$[\mathrm{ft}],[\mathrm{in}]$} \\
\hline $\mathrm{y}$ & Streamwise Position from Centerline & {$[\mathrm{ft}],[\mathrm{in}]$} \\
\hline$\alpha$ & Mach Angle & [deg], [rad] \\
\hline$\beta$ & Entrainment Ratio $=\dot{m}_{S} / \dot{m}_{P}$ & - \\
\hline$\gamma$ & Ratio of Specific Heats & - \\
\hline$\varepsilon$ & Nozzle Expansion Ratio $=\mathrm{A}_{\mathrm{exit}} / \mathrm{A}^{*}$ & - \\
\hline$\lambda$ & Slope Tangent to Characteristic Line & - \\
\hline$\rho$ & Density & $\left(\operatorname{slug} / \mathrm{ft}^{3}\right)$ \\
\hline$\theta$ & Streamline Flow Angle & [deg], [rad] \\
\hline
\end{tabular}

\section{Subscripts}

$0 \quad$ Stagnation Condition, Streamline Condition for Method of Characteristics

$1 \quad$ Known Property of Point 1 in Method of Characteristic Calculation

2 Known Property of Point 2 in Method of Characteristic Calculation

3 Known Property of Point 3 in Method of Characteristic Calculation

$4 \quad$ Method of Characteristics Point of Interest

$+\quad$ Positive Left Running Characteristic (Mach Line)

- $\quad$ Negative Right Running Characteristic (Mach Line)

$i \quad$ Arbitrary index denoting corresponding position

P Primary Stream

S Secondary Stream

\section{Superscripts}

* Critical Point (sonic throat) 


\section{Introduction}

Ejectors use a high velocity, high pressure flow to energize a low pressure low velocity flow. The high pressure driving flow is termed the primary flow. The low pressure flow being energized is the secondary flow. The area in which the primary and secondary flows interact is termed the mixing chamber or mixing duct. The conditions which dictate the interactions between the primary and secondary streams are many; however, the stagnation pressure ratio between the primary and secondary streams is the most referenced parameter. Since the purpose of an ejector is for the primary flow to entrain the secondary flow, the secondary to primary mass flow ratio, also called the entrainment ratio $(\beta)$, is a typical measure of ejector performance. Historically, ejectors have been used for industrial applications such as vacuum packaging, pumping chemical lasers, and thrust augmentation in aircraft turbine engines ${ }^{1}$. Ejectors are classified by their geometry, flow composition, and flow velocities.

\subsection{Geometry Classification}

Typical ejector geometries include axisymmetric, two-dimensional and lobed configurations. Axisymmetric ejectors have the primary and secondary streams concentrically arranged. Typically the primary flow is in the center and the secondary flow is entrained through an outer passage which is bounded by the primary nozzle and the duct wall. A basic axisymmetric ejector is shown in Figure 1-1.
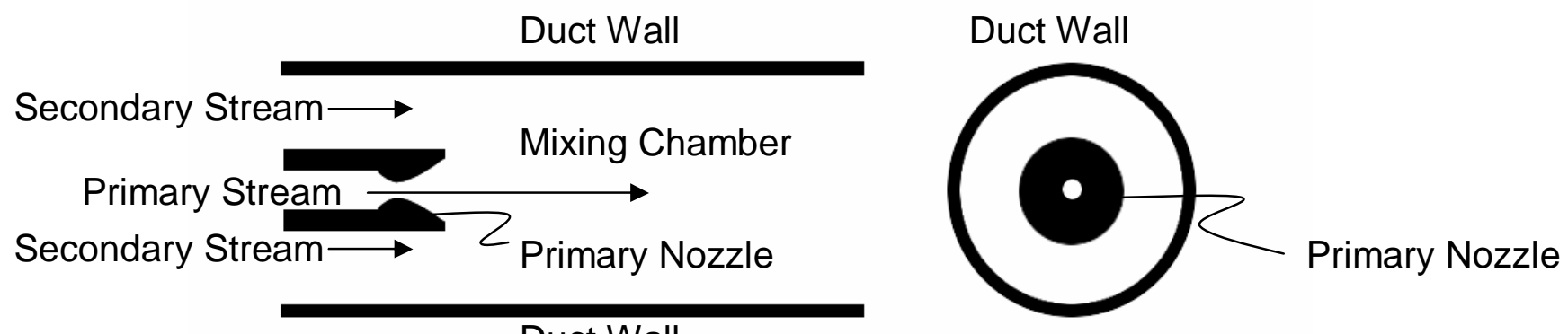

\section{Duct Wall}

Figure 1-1 Top and front view of an axisymmetric ejector

The top view of the axisymmetric ejector shows the duct walls extending far beyond the primary nozzle which is centrally located. The primary nozzle produces the high energy 
flow which entrains the fluid from the secondary stream. A long outer duct is required to promote complete mixing of the primary and secondary streams. The mixing chamber is the area bounded by the end of the primary nozzle in the stream wise direction and the end of the duct. The primary and secondary streams interact in the mixing chamber before exiting the ejector. The mixing chamber is also often called the mixing duct.

Two-dimensional planar ejectors generally have one line of symmetry on the centerline of the primary plume. A generic two-dimensional planar ejector is shown in Figure 1-2.
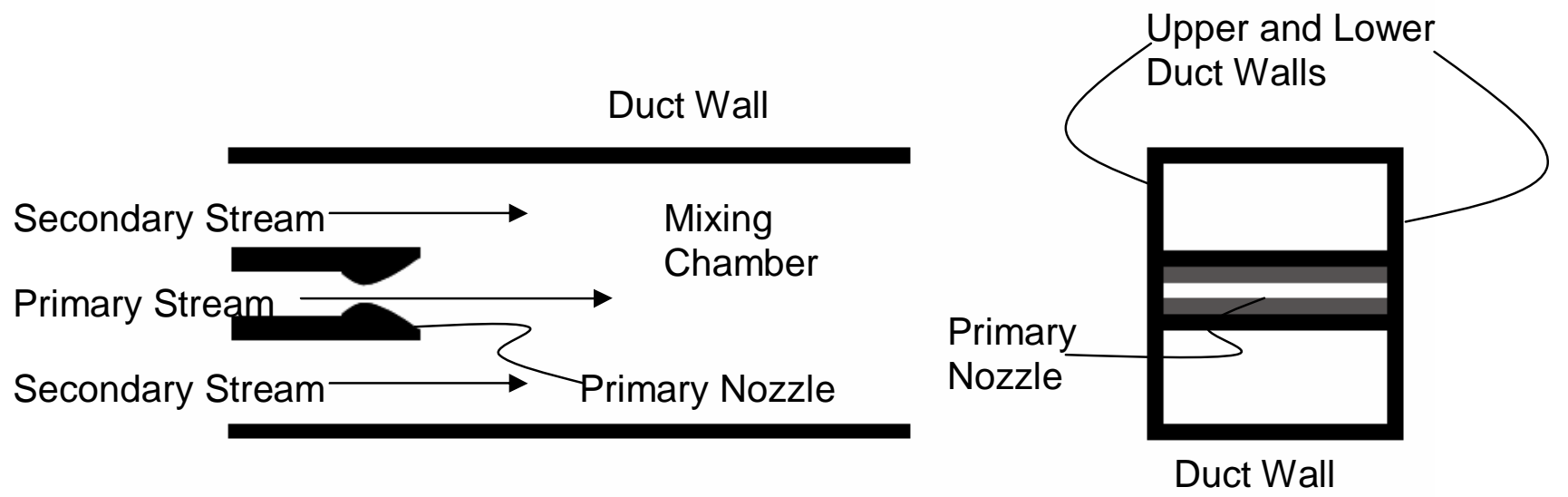

Figure 1-2 Top and front view of a two-dimensional planar ejector

Similar to the axisymmetric configuration, the primary nozzle is in the center, symmetrically entraining secondary flow. The primary and secondary flows react in the mixing chamber beyond the primary nozzle before exiting the duct. While the nozzle of an axisymmetric ejector is surrounded on all sides by the secondary flow; planar ejectors are bounded on the top and bottom by upper and lower duct walls. The primary nozzle extends from the lower duct wall to the upper duct wall. The secondary flow is not entrained above or below the primary flow. The planar configuration has reduced secondary flow area compared to axisymmetric ejectors of similar external dimensions. With the upper and lower walls acting as structure for planar ejectors, this geometry has configuration and packaging benefits. 
Lobed ejectors have flower shaped primary nozzles and various outer duct shapes. The purpose of the elaborate geometries is to promote mixing of the primary and secondary stream. Lobed ejectors may be axisymmetric or planar. A planar lobed ejector is shown in Figure 1-3.

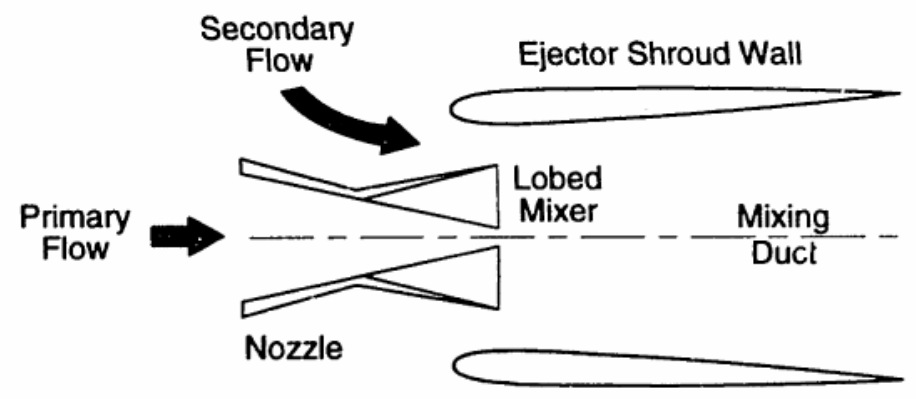

SECTION A-A

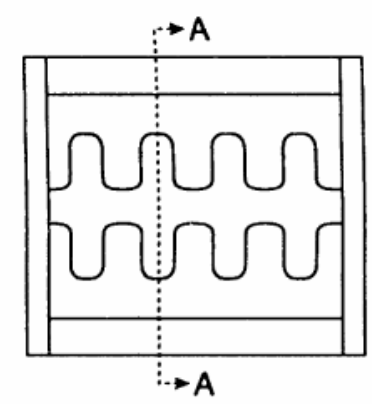

\section{Figure 1-3 Side and front view of a planar lobed ejector ${ }^{2}$}

The more elaborate primary nozzle geometry increases the surface area between the primary and secondary flows for more efficient mixing of the streams.

\subsection{Flow Composition Classification}

The gas properties of the primary and secondary streams have a large influence on the performance of an ejector. It is common to analyze ejectors which have primary and secondary streams of similar chemical composition. The basic air-air ejector analysis does not require consideration of chemical interaction. However, chemical and thermochemical reactions occur between the streams when the flows have different properties and compositions. These different flow compositions arise depending on the ejector application.

Changing the composition of the flows adds the complexity of chemistry based interactions between the primary and secondary stream. The streams may also be of different phase. The presence of liquid droplets or vapors can cause distinct flow phenomenon ${ }^{1}$. The amount of liquid present in the flow also influences the performance 
of the ejector and will change the optimal geometric configuration. Multiphase ejector analysis is important for heating and cooling applications.

An area of thermo-chemically reactive flow which has a propulsion application is that of a fuel rich combusting primary plume ${ }^{3}$. In this case there is an exchange of chemical, aerodynamic and thermal energy between the primary and secondary streams. The fuel rich primary plume is aided in combustion by the oxygen being entrained within the secondary flow. The transition from stored chemical energy to flow velocity achieved by this system makes it ideal for aerospace propulsion applications.

\subsection{Flow Velocity Classification}

Ejectors have primary or secondary flows which can be subsonic or supersonic. Subsonic ejectors such as induction pumps have lower primary to secondary stagnation pressure ratios. Neither the primary nor the secondary flow of a subsonic ejector ever achieves a sonic or supersonic condition.

Supersonic ejectors have higher primary to secondary stagnation pressure ratios. Choked flow in the throat of the primary nozzle due to a high chamber pressure is required to achieve supersonic primary flow. The primary flow accelerates to supersonic Mach numbers in the expanding area of the primary nozzle. The supersonic primary flow of an ejector is commonly referred to as the primary plume. The secondary flow velocity within a supersonic ejector varies. The secondary flow may enter at subsonic or supersonic Mach numbers. The secondary stream may exit the duct subsonic, sonic or supersonic, regardless of the inlet Mach number. The performance of the streams is determined by ejector geometry, primary to secondary stagnation pressure ratio, the ambient pressure at the ejector exit and the gas properties of the flows.

\subsection{Objectives}

The objective of developing the CPSE analysis method is to provide reliable and rapid approximations of a two-dimensional supersonic ejector with non-reacting flow of similar 
composition without empirical correction factors. The simulation provides insight into the relationship of stagnation pressure ratio and entrainment ratio within the ejector. The simulation will also give medium fidelity approximations of the properties within the primary and secondary flows. This tool can be used to run many cases before time intensive CFD or experimental analysis is performed for final high fidelity analysis.

The ejector serving as the topic for this analysis is two-dimensional planar in geometry. The primary and secondary flows are considered similar in gas composition and temperature. The similar flows do not have significant chemical interaction between the streams. The primary plume is high supersonic with flows up to Mach 5. The secondary inlet stream velocity varies from no flow to sonic. The primary and secondary streams do not have water droplets or condensation within the gases. Neither of the plumes undergo combustion at any stage of operation.

A computer automated analysis method has been developed to simulate a twodimensional planar air-air ejector for comparison with the Cal Poly Supersonic Ejector. This analysis tool is termed the CPSE simulation. The primary plume is described using the two-dimensional Method of Characteristics (MOC). The secondary stream is analyzed using isentropic relations. The interaction between the streams and the ejector surfaces are assumed to be inviscid. Thermo-chemical reactions between the streams are not considered.

The CPSE simulation is to be validated against experimental data obtained from the Cal Poly Supersonic Ejector experimental test rig. Recorded pressure ratio and entrainment ratio values are used as the standard for comparison. Once the simulation has been shown to correspond to this set up, many planar configurations can be simulated with the CPSE code within the given assumptions.

\subsection{Applications}

The primary application of interest for this study is the fusion of air augmented rocket technology with ramjet vehicles. A ramjet is a high speed propulsion technology which 
requires supersonic flight velocities in order to operate. Traditional reciprocating and jet engines use pistons and turbo-machinery to compress air to a level where it can be combusted. The combustion products then expand, creating mechanical energy or thrust. Ramjets do not require mechanical machinery for operation. Instead, the geometry of the ramjet inlet is designed to induce a shock train, terminating in a normal shock, which causes the air to decelerate from supersonic to subsonic speeds. Fuel is introduced into the subsonic high pressure air and combusted. The combustion products expand out of the ramjet nozzle supersonically, creating thrust. A subset of the ramjet system is the supersonic combustion ramjet, scramjet. The basic principles of ramjet operation hold true for scramjets; however, the incoming flow does not experience a normal shock and remains supersonic throughout the entire process. Figure 1-4 illustrates the fundamental ramjet propulsion cycle.

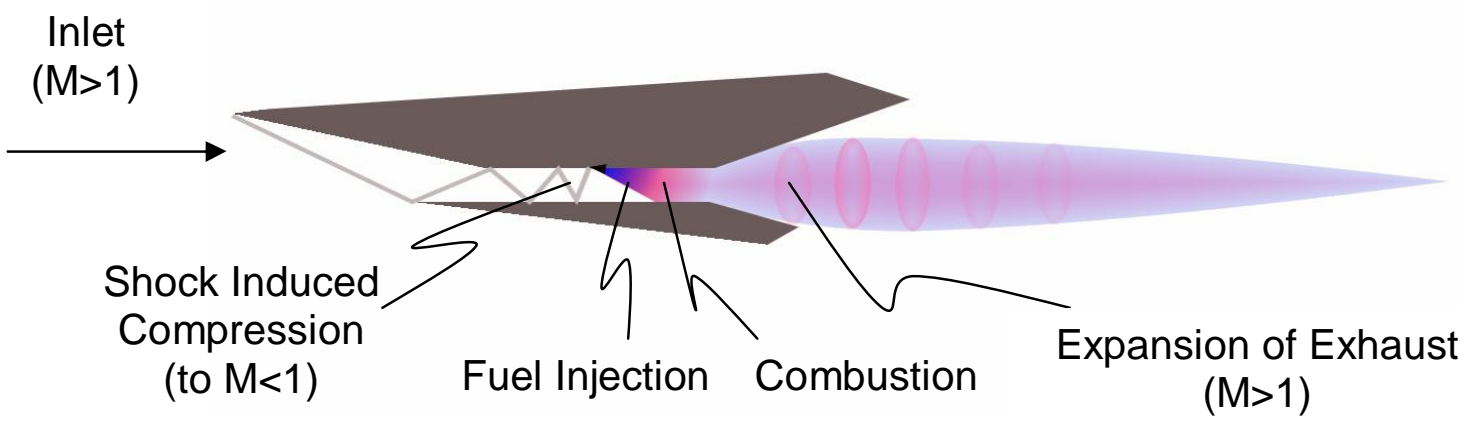

Figure 1-4 Ramjet operation cycle

Due to the high speeds required for ramjets to operate, their flight regime is limited. Figure 1-5 shows the variation of efficiency with Mach number for various propulsion systems. 


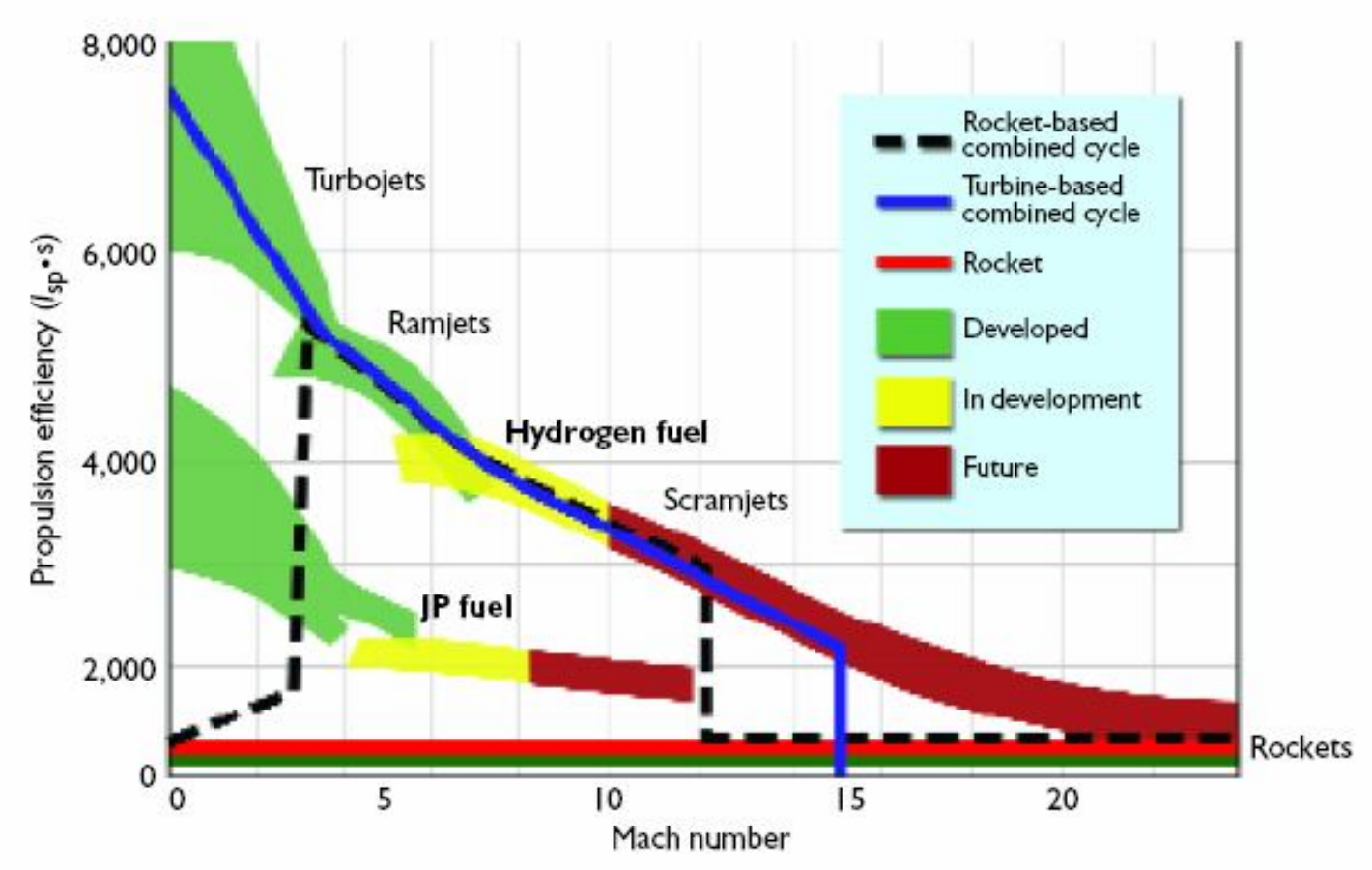

Figure 1-5: Effects of Mach number of efficiency of propulsion systems ${ }^{4}$

Figure 1-5 illustrates that there is a gap at low Mach numbers where a pure ramjet cannot operate. Turbojets are able to operate in the supersonic regime; though, the efficiency is greatly reduced beyond Mach 1 . Rockets are able to operate across the range of Mach numbers; yet, they operate very inefficiently in all conditions. Ramjets and scramjets are able to operate at Mach numbers beyond those of jet engines at higher efficiencies than rockets. However, in order to operate a ramjet, the system must be accelerated to supersonic speeds. Ramjets operate most efficiently between Mach 2.0 and Mach 5.04. Vehicles operating beyond Mach 5 may be accelerated by auxiliary booster vehicles or utilize combined cycle systems. Combined cycle propulsion systems use turbojets or rockets to accelerate hypersonic vehicles to a flight condition where the ramjet cycle can operate.

The X-43 scramjet test vehicle shown in Figure 1-6 was launched from a modified Pegasus missile which had been dropped off a B-52 in order to achieve the required flight conditions for operation. 


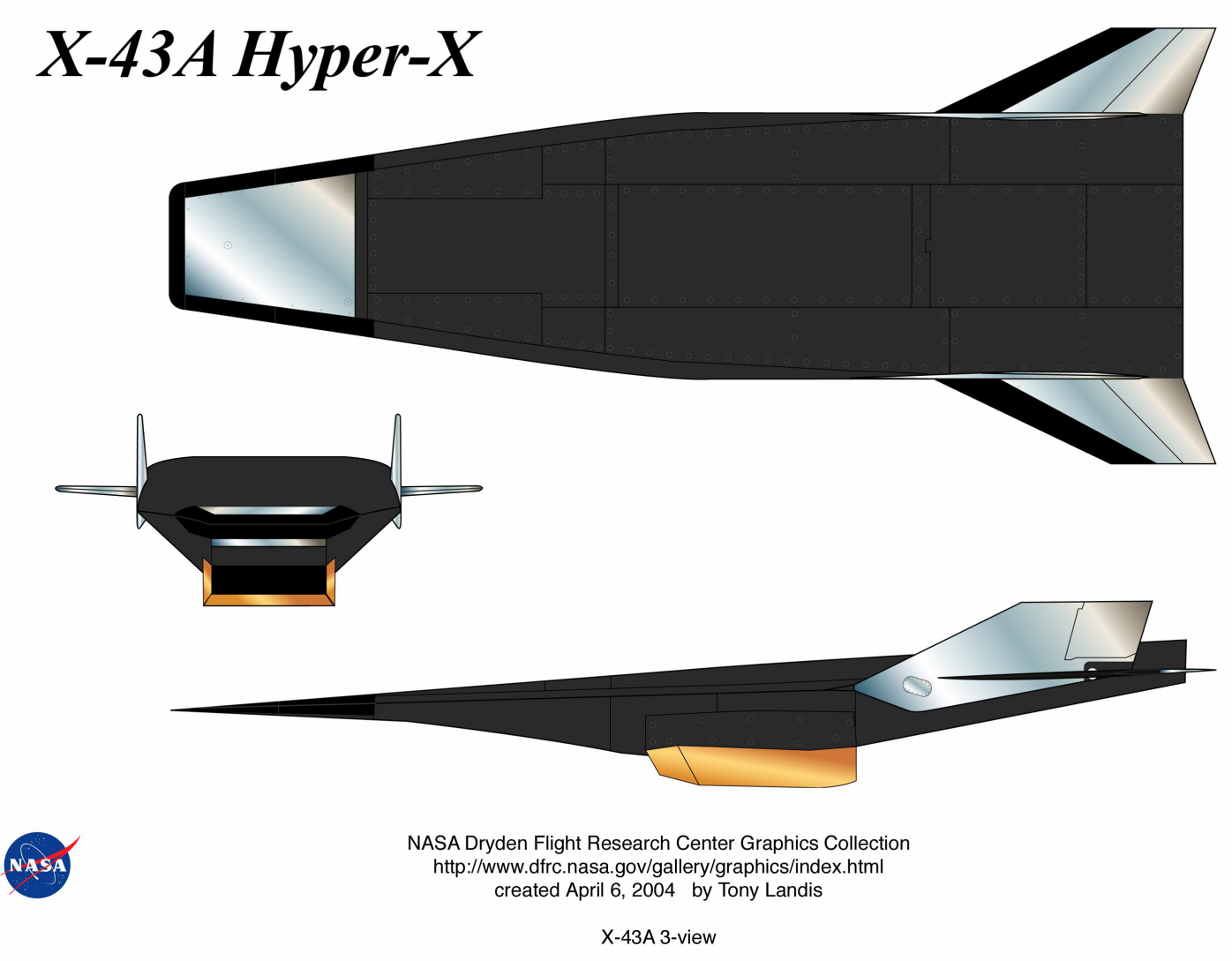

Figure 1-6 X-43 Hypersonic test vehicle 3-view ${ }^{5}$

The X-43 is an example of a multi-stage vehicle. The B-52 which lifted both the Pegasus missile and the $\mathrm{X}-43$ is considered the first stage of the system. The Pegasus which dropped off the wing of the B-52 and accelerated the X-43 to operating speeds was the second stage. The X-43 itself became the final stage once it departed from the Pegasus. A schematic of the multistage operation of the $\mathrm{X}-43$ system is shown in Figure 1-7. 


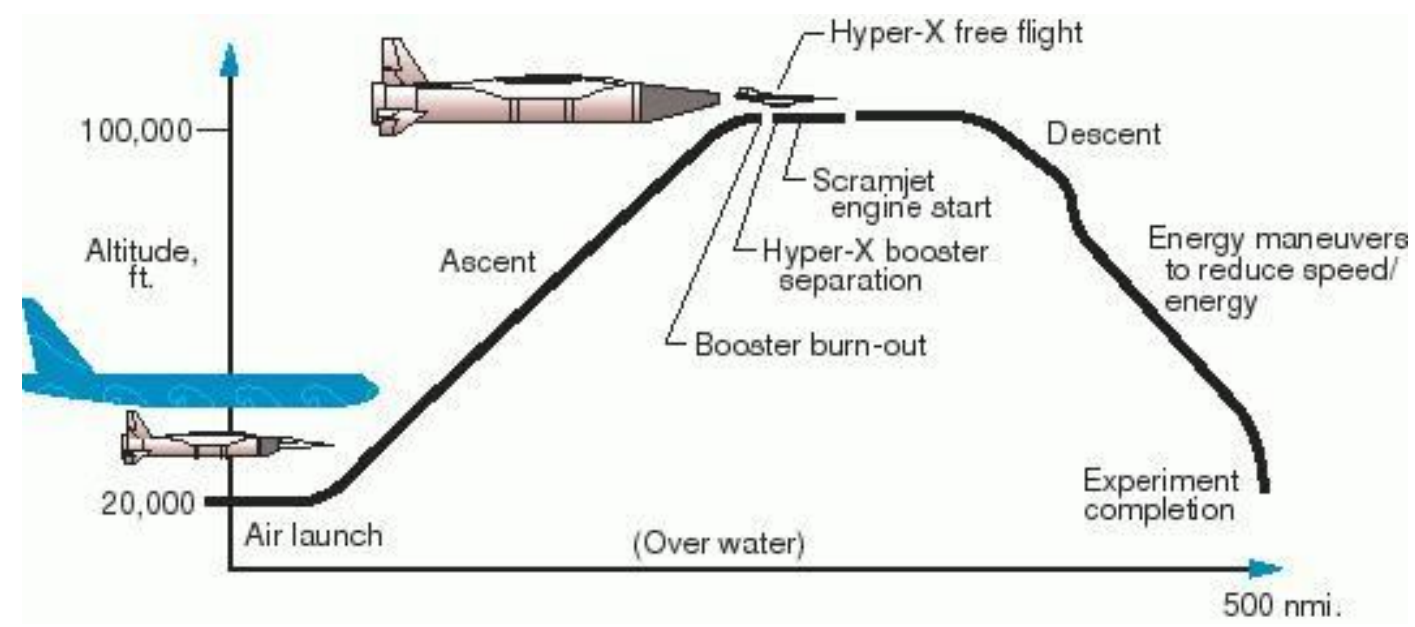

Figure 1-7 Schematic of the multistage operation for the X-43 test flight ${ }^{6}$

The SR-71 Blackbird aircraft shown in Figure 1-8 has engines which switch to ramjet propulsion from jet propulsion at high Mach numbers. The SR-71's J58 turbo-ramjet engines are an example of a combined cycle propulsion system.
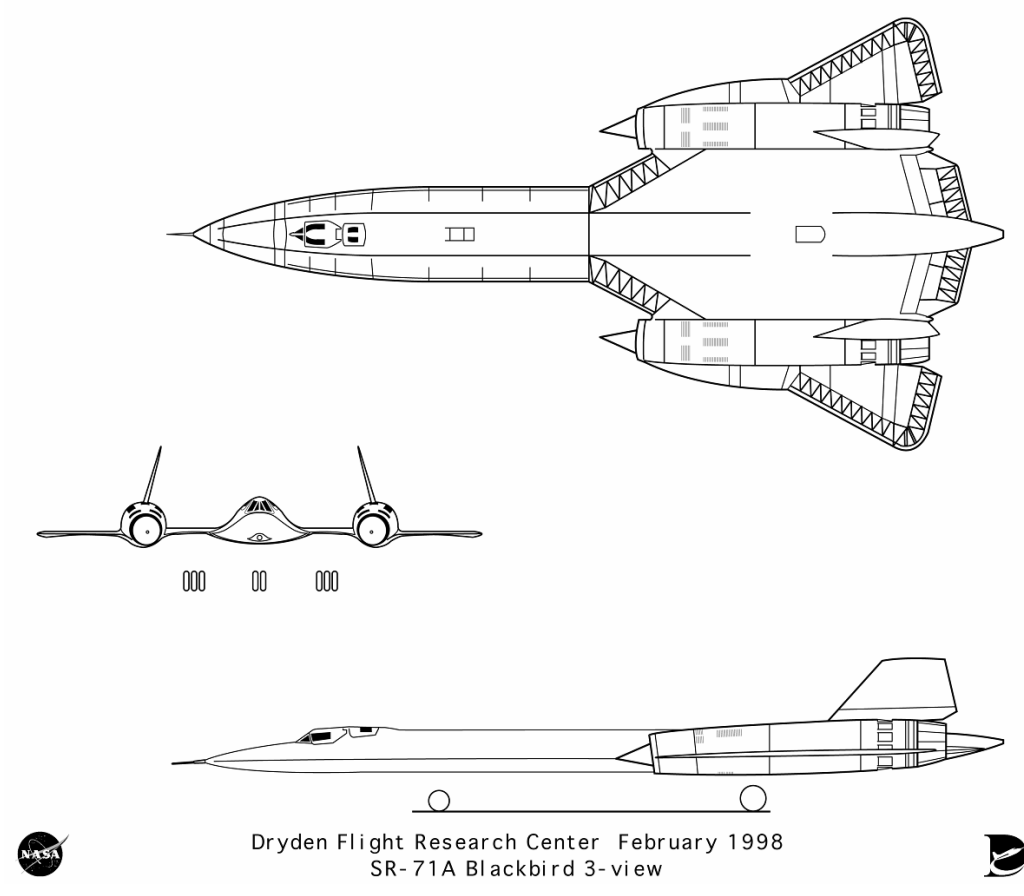

Figure 1-8 SR-71 turbine based combined cycle vehicle 3-view ${ }^{7}$

The SR-71aircraft takes off and accelerates using jet engines. Once the vehicle switches to ramjet mode, no components of the system are dropped or discarded. The vehicle can then switch back to turbojet mode for lower flight speeds and landing. This integrated combined cycle propulsion system is an example of a turbine based combined cycle, 
TBCC. TBCC systems incorporate the high efficiency of turbine engines at low Mach numbers and the ability of Ramjets to operate at high Mach numbers.

Recently TBCC systems have received renewed attention. Lockheed Martin responded to Darpa's Falcon program request for a hypersonic flight vehicle with horizontal takeoff and landing capability with the Blackswift hypersonic cruise vehicle (HCV). Blackswift utilized shared inlets and nozzles for the turbine and ramjet propulsion systems. The demonstrator would have been able to takeoff from a runway, accelerate to Mach 6 under its own power and maneuver at hypersonic speeds before landing. It was reported that the required funding was not supplied and the project was cancelled by the year 2009 . The Falcon program is continuing structural and aerodynamic development for hypersonic vehicles using unpowered gliders launched with Minotaur booster rockets ${ }^{8}$.

\section{A NASA concept designed to test TBCC systems is shown in Figure 1-9. TBCC} systems are limited in that they cannot operate at very high Mach numbers or at very high altitudes due to lack of atmospheric density which is required to operate air-breathing propulsion systems. These restrictions limit space and orbital related applications of TBCC systems.

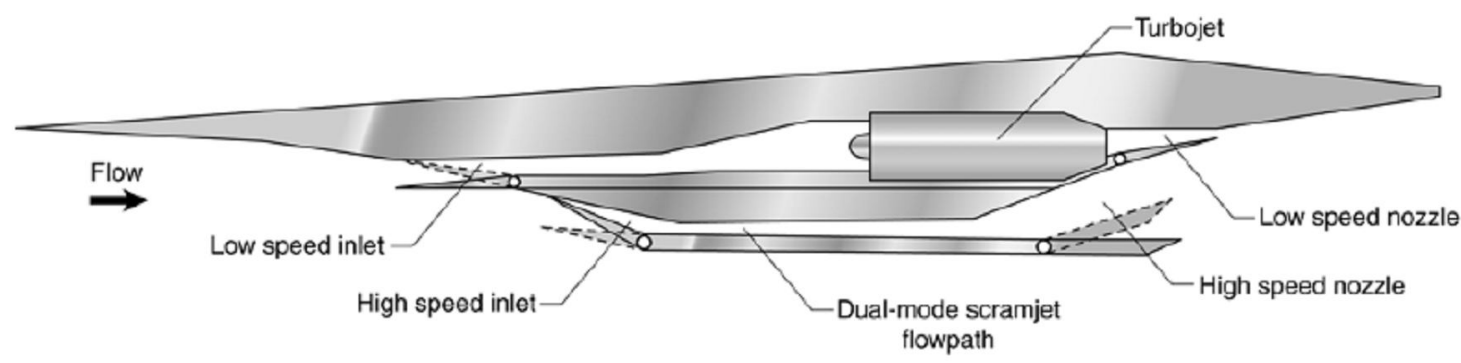

Figure 1-9 Turbine based combined cycle engine integration'

Currently, vehicles such as the Space Shuttle require multiple stages to reach Earth's orbit. These rocket based systems require multiple stages because the fuel and the oxidizer for all the stages must be carried in large tanks which are discarded throughout the mission. By reducing the oxidizer storage requirements, it may be possible to create a vehicle that requires only a single stage to orbit, SSTO. 
As an alternative to staged vehicles and turbine based combined cycles, rocket based combined cycles (RBCC) offer a viable solution for SSTO. At supersonic and low hypersonic speeds, the RBCC operates as a ramjet or scramjet. When the vehicle exceeds the altitude or Mach envelope of ramjet operation, the vehicle is powered by a traditional rocket. At low speeds the vehicle operates in air augmented rocket (AAR) mode until it is able to make the transition to ramjet mode. AAR mode differs from pure rocket mode in that the inlet to the engine remains open and air is entrained around the rocket plume to provide additional thrust. A conceptual RBCC vehicle is shown in Figure 1-10.

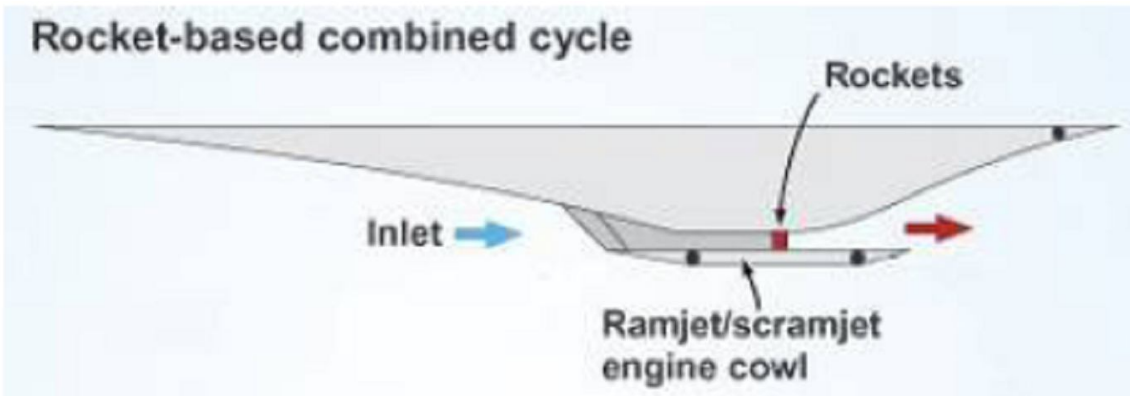

Figure 1-10 Concept vehicle incorporating a rocket based combined cycle propulsion system ${ }^{10}$

An AAR is an ejector which operates with a supersonic combusting primary plume. The most thrust augmentation is gained when the configuration operates in the saturated supersonic mode with a fuel rich primary plume. As the pressure ratio between the rocket chamber and ambient air is increased, the Fabri-limit is exceeded and the amount of entrained air is decreased. As the pressure ratio is increased further, the secondary flow may become blocked. Performance is significantly decreased in the blocked case; the engine may be damaged in extreme conditions.

Research suggests axisymmetric ejectors result in increased thrust augmentation compared to two-dimensional planar configurations ${ }^{11}$. However, recent configuration trade studies have emphasized the need for increased packaging efficiency, interchangeability and modularity in RBCC propulsion options ${ }^{4}$. Planar geometries of AARs offer these configuration benefits over axisymmetric configurations. 
It is imperative to understand the conditions which lead to optimum AAR performance and how to avoid reduced performance and engine damage. In the following sections the operation of planar ejectors across a range of pressure ratios is examined to better understand the factors which determine the performance of air augmented rockets.

\section{Literature Review}

Countless works have been compiled regarding the interactions occurring within supersonic air to air ejectors. These works have provided knowledge, methods and motivation for the analysis to follow.

\subsection{Seminal Works}

Fabri $^{12,13}$ defined the operating conditions of an air-air ejector with supersonic primary flow which causes aerodynamic choking of the secondary flow. Emanuel ${ }^{18}$ compared $^{2}$

Fabri's method with the one-dimensional method and proposed a hybrid method. Addy ${ }^{14}$ expanded on Fabri's method, introducing various degrees of viscous interaction and a transient analysis. These methods share the trait that they evaluate ejector performance by total analysis from inlet to exit.

\subsubsection{Fabri}

Fabri et al ${ }^{12,13}$ systematically investigate operating conditions of an air to air jet ejector with high pressure supersonic primary flow and low pressure induced secondary flow. The configuration used for the analysis is cylindrical and axisymmetric. Fabri defines several aerodynamic flow patterns of ejector operation in order of decreasing primary stagnation pressure.

Fabri's analysis is of a cylindrical ejector with the primary nozzle aligned with the cylinder axis. This analysis does not take into account viscosity or diffusion between the streams. A correction is made for the friction between the secondary flow and the duct wall. The primary and secondary flows are assumed to be the same gas which is treated as a perfect gas. The primary flow is low supersonic at the exit of the primary nozzle. The velocity of secondary flow at the entrance to the mixing chamber varies. At the exit of the mixing chamber, the flows have uniform pressure which matches the exit condition. 
The primary flow is solved using the classical quasi-one-dimensional approach.

However, Fabri suggests that the Method of Characteristics be utilized when the primary plume area is expanding because the quasi-one-dimensional approach requires correction factors to predict the area of the primary plume. The values of the inlet conditions to the mixing chamber of the primary and secondary streams are used to solve the conservation equations. The outlet condition is the sum of the primary and secondary inlet mass flow, momentum and energy with the condition of uniform ambient pressure imposed.

Although the interaction between the streams is inviscid, a correction for pressure loss due to wall friction is imposed. Fabri's analysis provides some insight into the flow phenomenon occurring within the ejector from experimental trials. Fabri's analysis method does not yield the properties of the streams within the flow. Fabri defines three flow patterns which classify different regimes of supersonic ejector operation. The flow may be termed Fabri choke supersonic, saturated supersonic or subsonic. The Fabri choke and saturated conditions are both special cases of the supersonic case. If the duct is suffiently short the mixed case may occur where the secondary stream does not achieve aerodynamic choking before the duct exit.

At the lowest primary stagnation pressure which still produces sonic flow, the flow pattern is classified by the Fabri choke supersonic case in which the primary flow is supersonic and the secondary flow achieves a sonic condition due to the expanding primary plume which acts as an aerodynamic throat in the mixing chamber. Weak shocks and expansion fans are likely to exist within the primary plume. However, this shock structure is not significant enough to decelerate the plume to a subsonic Mach number. This condition is often referred to as the Fabri choke condition and is displayed in Figure 2-1. 


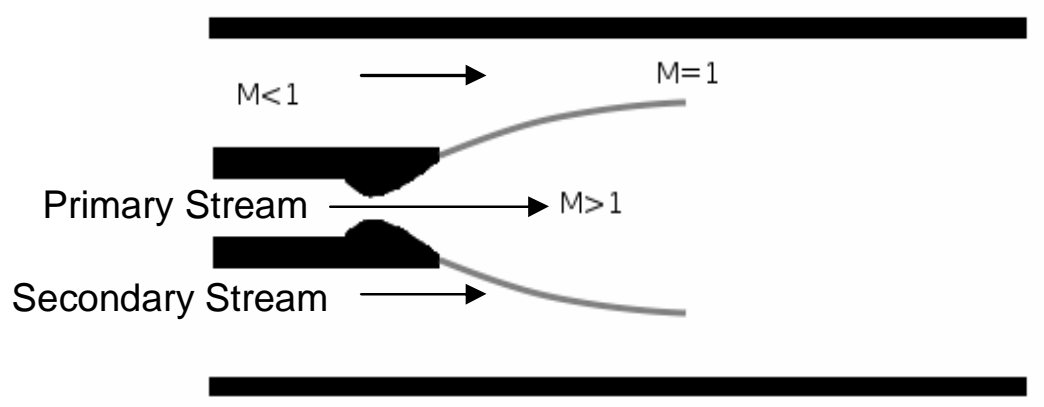

Figure 2-1 Secondary flow achieves critical Mach number during the Fabri choke condition

When the primary stagnation pressure is increased the flow pattern becomes saturated supersonic. The saturated supersonic case occurs when the secondary flow achieves aerodynamic choking and a sonic condition in the secondary duct before being exposed to the primary plume. This occurs due to a minimum geometric area between the duct wall and the primary nozzle. The primary plume remains supersonic; however the plume does not expand. Weak shocks are likely to exist within the primary plume. The saturated flow pattern is shown in Figure 2-2.

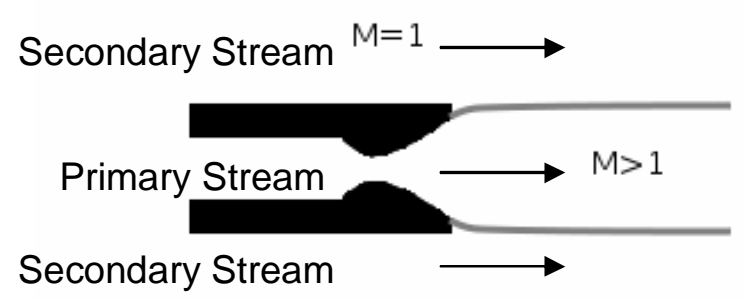

Figure 2-2 The secondary flow entering the mixing chamber is sonic during the saturated flow pattern 
The flow pattern with the lowest primary stagnation pressure which still induces secondary flow is the subsonic case. The subsonic condition occurs when the primary pressure is low enough that the back pressure from the ambient exit condition forces a strong shock train to form in the primary plume which terminates in a normal shock. This shock structure decelerates the primary flow to subsonic velocities. The secondary flow is then entrained by a subsonic primary flow and the streams become fully mixed before exiting the duct. This is illustrated in Figure 2-3.

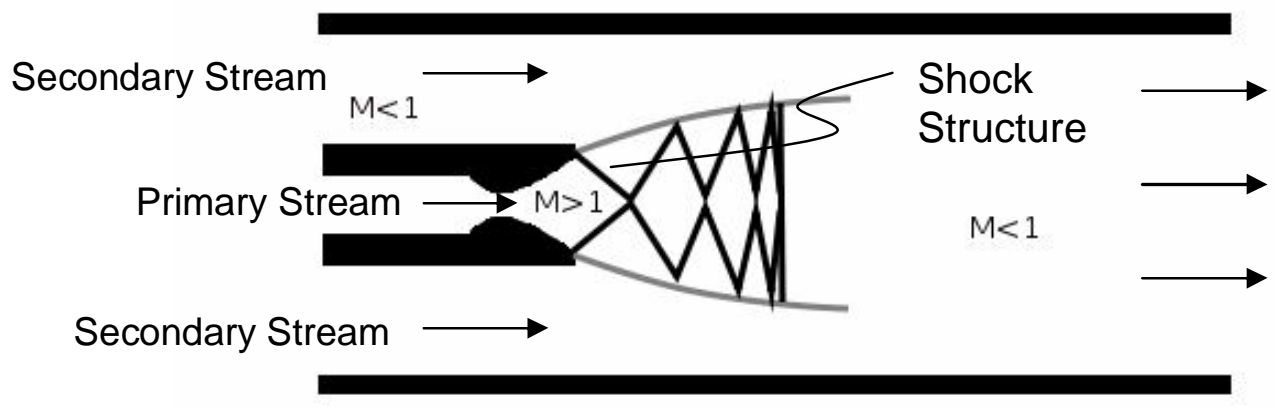

Figure 2-3 The primary flow becomes subsonic during the subsonic flow pattern Fabri's method utilizing conservation of energy and momentum to solve for the aerodynamic flow patterns for each condition provides some insight into the interactions between the flows. However, Fabri's method does not accurately provide properties of the flow within the mixing chamber. Although the method is inviscid, corrections are made for the friction from the duct walls and the thickness of the lip of the primary nozzle. Later analyses ${ }^{2,3,11,14,15,16}$ reject the nozzle lip thickness correction and implement a mixing layer between the primary and secondary streams.

\subsubsection{Emanuel}

Emanuel ${ }^{18}$ compares the one-dimensional analysis of supersonic air to air ejectors to Fabri's inviscid method. The one-dimensional calculations are typically used for parametric analyses due to the ease of implementation. In this method, all parameters are fixed, besides a single independent variable, typically the inlet Mach number. The flows are mixed in a constant area mixer. The ejected flows may be supersonic, or 
subsonic. The one-dimensional method provides little insight into the flow phenomenon occurring within the ejector. Due to the amount of properties required to run the calculations, a large number of assumptions and apriori knowledge is required. Accurate properties of the streams within the mixing chamber are not provided by this method.

The one-dimensional ejector model requires the assumption of constant area mixing or constant pressure mixing. It is possible for both assumptions to be applied. The constant area assumption requires the mixing area to remain unchanged in the stream wise direction. The constant pressure assumption implies the primary and secondary pressures are equal entering the mixing chamber. Further, it is assumed that the primary and secondary flows are fully mixed at the exit of the ejector duct.

The primary and secondary flows are characterized by their stagnation conditions as well as the Mach number and area at the entrance to the mixing chamber. From these values, properties such as velocity and mass flow rate can be calculated. A control volume approach with conversation of mass, momentum and energy is used to find the final solution ${ }^{19}$. This method is used for subsonic and supersonic exit velocities. The subsonic case is calculated in the same way as the supersonic case; however a normal shock is imposed in the stream to decelerate the flow before exiting the duct.

In order for Emanuel to compare the Fabri method to the 1-D method, the assumptions of both the 1-D method and the Fabri method must be imposed. Due to the large number of assumptions, the solution domains and implications of this comparison are limited. The primary conclusion of Emanuel's comparison is that Fabri's isentropic 1-D based method has many limitations. The main criticism is that Fabri does not mention or rule out cases where the incoming secondary flow is supersonic. Recall, the maximum achievable flow rate of the secondary stream discussed by Fabri occurs in a saturated case where the flow becomes choked in the secondary duct before being exposed to the primary plume. However, supersonic-supersonic ejectors have been investigated by various sources ${ }^{20}$. Emanuel also states that Fabri's isentropic method of describing the primary flow breaks down when the secondary flow enters the mixing chamber at transonic speeds. Emanuel 
suggests that this issue may be remedied by solving for the primary plume area using the Method of Characteristics. A more important conclusion from Emanuel's work is that combination or hybrid methods of ejector analysis may be tailored to obtain results with various levels of fidelity and utility.

\subsubsection{Addy}

Addy ${ }^{14}$ presents perhaps the most comprehensive analysis of axisymmetric air to air ejectors with supersonic primary plume. Addy starts by imposing many familiar assumptions in his analysis. The geometry of the ejector is axisymmetric and cylindrical. The primary and secondary flows are of the same perfect gas composition with the same stagnation temperatures. The primary flow is supersonic at the exit of the primary nozzle. The secondary flow velocity varies. The Mach number is uniform at the exit of the duct.

Addy then extends Fabri's analysis by utilizing the Method of Characteristics to describe the primary plume. Addy also adds the capability to quantify the viscous interaction between the primary and secondary streams. Addy then presents a method of transient ejector analysis.

The Method of Characteristics acts as a base for Addy's method of analysis. Use of the Method of Characteristics provides a two-dimensional distribution of the gas properties of the primary plume. The Method of Characteristics also yields much higher quality predictions of the primary plume than one-dimensional and quasi-one-dimensional estimates. The pressure along the boundary of the primary plume determined by the Method of Characteristics and the secondary stream analysis must be continuous. The one-dimensional secondary stream properties are solved using the primary plume shape with a guess for the inlet Mach number and the ratio of primary stagnation pressure to secondary inlet pressure. The condition of continuous pressure along the interface between the primary and secondary streams assures the flows are compatible. With a physically possible solution calculated after each trial, the inlet Mach number is then adjusted after each run until the desired solution is attained. Addy focuses on the 
supersonic Fabri choke condition in which the secondary stream achieves a sonic condition in the mixing chamber due to expansion of the primary plume.

Addy's analysis begins with the various steady state cases discussed by Fabri. The first cases discussed are the saturated supersonic condition and supersonic Fabri choke condition which operate independent of ambient to primary pressure ratio. The Method of Characteristics is used to determine the minimum area available for the secondary flow given a guess for the secondary inlet Mach number. For this analysis, the secondary stream may remain subsonic, may achieve a sonic condition before the minimum area, or may become sonic at the minimum area of secondary flow. If the secondary flow does not achieve the sonic condition at the minimum area, the assumed Mach number of the secondary inlet must be changed until the results match the desired properties.

Each final solution provides the secondary to primary mass flow ratio and the secondary to primary stagnation pressure ratio. The process also yields the properties of the primary and secondary streams within the mixing chamber which the Fabri and one-dimensional method cannot. Use of the Method of Characteristics provides the jet boundary location of the primary plume, the angle of the boundary between the primary and secondary flows, and a two-dimensional Mach number distribution within the primary plume. The analysis of the entrained flow yields the quasi-one-dimensional Mach number and pressure distribution of the secondary stream. Addy presents methods for inviscid solutions, as well as viscous superposition corrections. A full viscous solution is also presented. Following the discussion of a full viscous solution, the effects of ambient to primary pressure ratios are investigated.

Addy finishes his steady state ejector analysis with an example of parametric solution surfaces and a comparison of steady state ejector analysis methods. Before the analytical approximations are compared to experimental results, Addy discusses the topic of transient operation which is based on characteristic times. The characteristic time is a function of the ejector geometry and the speed of sound. Addy reports that the 
correlations between analytical and experimental results are acceptable for steady state conditions and "indistinguishable" for transient conditions.

Addy paves the way for high fidelity ejector analysis by addressing issues such as non cylindrical ducts, full characterization of the primary and secondary flows and viscous interaction. Since the work of Addy many studies have focused on further increasing the fidelity of the interactions within supersonic ejectors.

\subsection{Supplemental Analysis Approaches}

Since the work of Addy in 1963, the fidelity of analysis of interacting flows has been bolstered by increased focus on specific flow phenomena and the development of new methods. Shear layers, reacting flows, abstract geometries and CFD methods are some of the many topics which can be applied to ejector analysis.

\subsubsection{Shear Layers}

The concept of viscous mixing between tangential flows is a topic which has been investigated extensively ${ }^{2,3,16,21}$. Hall, Dimotakis and Rosemann ${ }^{15}$ use Schlieren photography to validate analytical approximations of turbulent shear layer growth in non reacting flow.

Popamoschu ${ }^{11}$ investigates mixing in planar and axisymmetric ejectors to examine thrust augmentation. Analytical equations are developed for heat transfer and turbulent shear layers in ejectors with supersonic primary plumes and subsonic entrained flow. The primary and secondary streams are analyzed as quasi 1-D flows of air. The effects of mixing and heat transfer from the analytical equations are transformed into a local coordinate axis and superimposed along the streamline separating the primary and secondary streams. Popamoschu concludes that axisymmetric configurations outperform two-dimensional planar ejectors due to reduced skin friction between the secondary stream and the duct walls. He also concludes that thrust augmentation benefits of ejectors become "nil" when the incoming Mach number of the secondary flow reaches 0.7 . 


\subsubsection{Reacting Flow}

The mixing of reacting flows is explored by various sources ${ }^{16,21,22}$. Cutler et $\mathrm{al}^{3}$ investigate the chemical, thermal and aerodynamic mixing of a supersonic combusting jet with coflow into the ambient free stream. Cutler's primary focus is to provide a basis for validation for Computational Fluid Dynamics (CFD) trials. Cutler aims to use the experimental observations to serve as a standard to evaluate the combusting turbulent mixing predictions of the Navier-Stokes equations. Beyond visual observations, Cutler also records temperature and composition of the flows due to mixing using the nonintrusive coherent anti-stokes Raman spectroscopy (CARS) technique. Although the jet and the coflow mix the ambient air instead of a duct, the reactions and shear layer profiles of supersonic and subsonic flow are analogous to that of a supersonic ejector. The flow visualization revealed that as the Mach number of the primary plume increased, the combustion moved from the nozzle to further down stream. Also, coflow combustion greatly increases the plume width compared to non combusting flow.

\subsubsection{Lobed Ejectors}

While most work on ejector theory and experiments pertains to axisymmetric or planar configurations, Andrew Kang Sang Fung ${ }^{2}$ explores mixing due to the effects of varied ejector geometry. His work includes comparisons of analytical approaches and numerical Navier-Stokes solutions against experimental data. Ultimately a model is developed to predict mixing, performance and losses in lobed ejectors.

\subsubsection{Computational Fluid Dynamics}

CFD analysis is often used to study the mixing of flows in supersonic ejectors of various configurations ${ }^{2,21}$. Grosch, Seiner, Hussaini and Jackson ${ }^{16}$ utilize the 3-D Navier-Stokes equations to investigate the effects of tabs on the mixing of high speed hot flow into a lower speed cold flow. The study covers three main areas. The first topic investigated is the mixing of flows in an undisturbed duct. The influence and proper utilization of tabs to increase mixing rates is then explored. Finally, the actual phenomena which facilitate the mixing of the streams are examined. The study concludes that the tabs induce vortices which cause the high momentum hot primary jet stream to energize the low temperature low energy induced stream. Configurations consisting of up to six tabs were shown to increase mixing. 


\subsection{Motivational Works}

The analysis, fabrication and experimental investigation of a two-dimensional planar Cal Poly Supersonic Ejector at the California Polytechnic State University has been carried out by Foster and Gist. The purpose of these experiments is to investigate entrainment properties in planar air augmented rockets. The works of Foster ${ }^{10}$ and Gist ${ }^{4}$ are the primary motivation for this research. Foster operated the CPSE with hot flow, in which the primary flow undergoes combustion in the primary chamber. Gist operated the CPSE with cold flow, during which no combustion occurs. Both experiments entrain atmospheric air as the secondary stream and discharge back into the ambient air from the ejector exit.

\subsubsection{Foster $^{10}$}

Trevor Foster's trials with the Cal Poly Supersonic Ejector shown in Figure 2-4 were tested with a hot primary plume. Although the chamber pressure is driven by combustion, it is critical to note that the primary plume is not fuel rich in these trials. Foster uses an oxidizer to fuel mixture ratio of 2 . The combustion process is complete before the flow exits the primary nozzle. Four different primary pressures were tested. The primary flow was methane and oxygen. The secondary flow was air entrained from ambient conditions. 


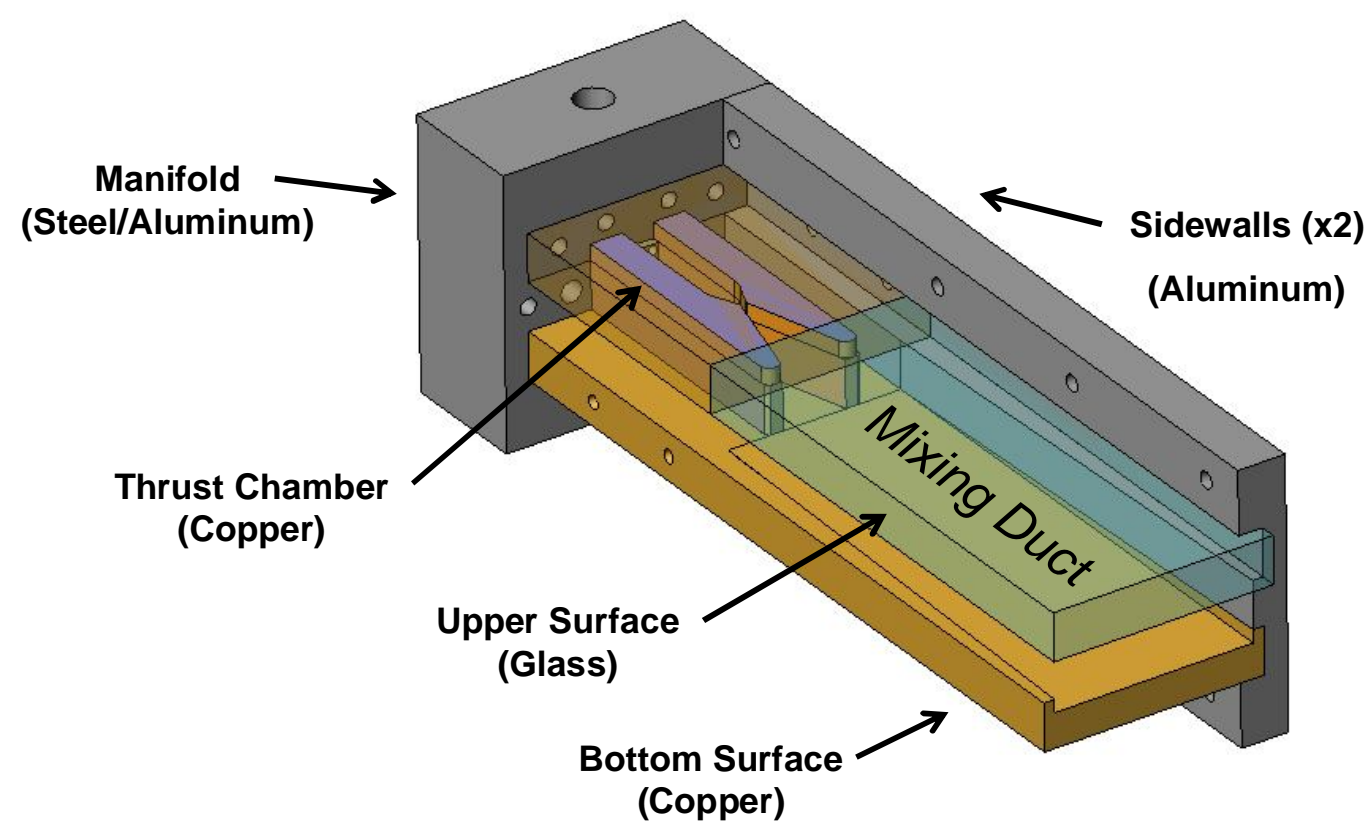

Figure 2-4 The Cal Poly Supersonic Ejector

Foster developed the ducted rectangular two-dimensional symmetric thruster powered by methane and oxygen, the Cal Poly Supersonic Ejector. The principal phenomenon of interest is the expansion of the primary supersonic plume and its interaction with the flow being entrained from ambient. Foster varies the primary stagnation pressure from 325 to 1032 pounds per square inch; achieving a maximum pressure ratio of 74 . Foster suggests this case is in the supersonic regime near the Fabri limit; however the experimental apparatus is not able to achieve pressure ratios high enough to reduce the secondary entrainment. A reduction in entrainment is required to prove the Fabri limit maximum entrainment has been achieved. Foster concludes that cold flow runs with a nitrogen primary stream with higher pressure ratios are capable of entraining more air than the methane-GOX hot fire tests. Foster also observes that the stream-wise location of the minimum area of the secondary flow is constant, independent of the pressure ratios and flow velocities. Foster uses Fabri's isentropic one-dimensional analysis with correction factors for nozzle thickness and non isentropic expansion for his theoretical predictions. High Definition video cameras are used for visualizing and recording the flow within the 
ejector. Thermocouples and pressure transducers are used for quantitative measurement of the flow within the ejector.

\subsubsection{Gist $^{4}$}

Gist extends the capability designed for Foster's experiments with modified nitrogen tanks to allow higher primary stagnation pressures which lead to higher overall pressure ratios. These trials with the increased pressure ratio capability are conducted with a cold primary flow. The secondary flow of air is assumed to be of similar composition to the primary flow.

Gist focuses on the effect of stagnation pressure ratio on entrainment ratio. Gist also investigates which pressure ratios will produce the phenomenon known as Fabri choking, the aerodynamic choking of the secondary stream in the mixing chamber caused by the expansion of the supersonic primary plume. Gist also hypothesizes that at very high pressure ratios the primary plume will expand out to the duct walls, blocking the secondary flow. Gist was not able to achieve pressure ratios high enough to yield the blocked flow pattern.

By modifying the test rig designed by Foster, Gist increases the cold-flow operating pressure ratios in the two-dimensional planar Cal Poly Supersonic Ejector. Gist is able to achieve primary stagnation pressures up to 1690 pounds per square inch. With the higher chamber pressures Gist reports mixed and supersonic Fabri choke aerodynamic flow patterns. The highest entrainment levels occur at the transition between Fabri choke and saturated supersonic conditions as predicted by Fabri. With the high primary stagnation pressure, Gist observes primary plume Mach numbers as high as 3.92. The high primary Mach number and the two-dimensional planar configuration of the ejector are what set Gist apart from classic ejector analysis with are typically axisymmetric with a low supersonic or sonic primary flow. Gist uses Fabri's one-dimensional isentropic analytical approximation with an empirical correction to account for the two-dimensional shock structure necessary to predict the saturated and Fabri choke conditions. His predictions match experimental entrainment ratios within $12 \%$. Gist also makes an attempt to characterize the shock structure within the primary plume. However, the flow 
visualization technique of high definition video is not a definitive method of verifying the predicted shock structure.

Gist and Foster use similar analysis approaches to predict the experimental results of the two-dimensional planar ejector. The primary plume is calculated using the onedimensional inviscid analysis. The entrainment ratios are found using Fabri's saturated flow calculation in Equation 2.1. The entrainment ratio $(\beta)$ is a function of the secondary choking area $\left(A_{S}^{*}\right)$, secondary stagnation pressure $\left(P_{0 S}\right)$, primary nozzle throat area $\left(A_{P}^{*}\right)$ and the primary chamber pressure $\left(P_{0 P}\right)$. This formulation of the entrainment ratio is derived from the saturated condition which sets the secondary choking area equal to the area of the secondary flow inlet. This area is later adjusted using an empirical correction factor for growth of the primary plume.

$$
\beta=\frac{A_{S}^{*} P_{0 S}}{A_{p}^{*} P_{0 p}}
$$

Equation 2.1

A correction factor is implemented to account for the thickness of the base of the primary nozzle $\left(t_{b}\right)$ as suggested by Fabri. A second empirical correction factor takes into account the change in area of the primary plume when it does not undergo ideal expansion. The presence of shocks in the primary plume causes variation in the pressure distribution and an increased plume area as shown in Figure 2-5. 

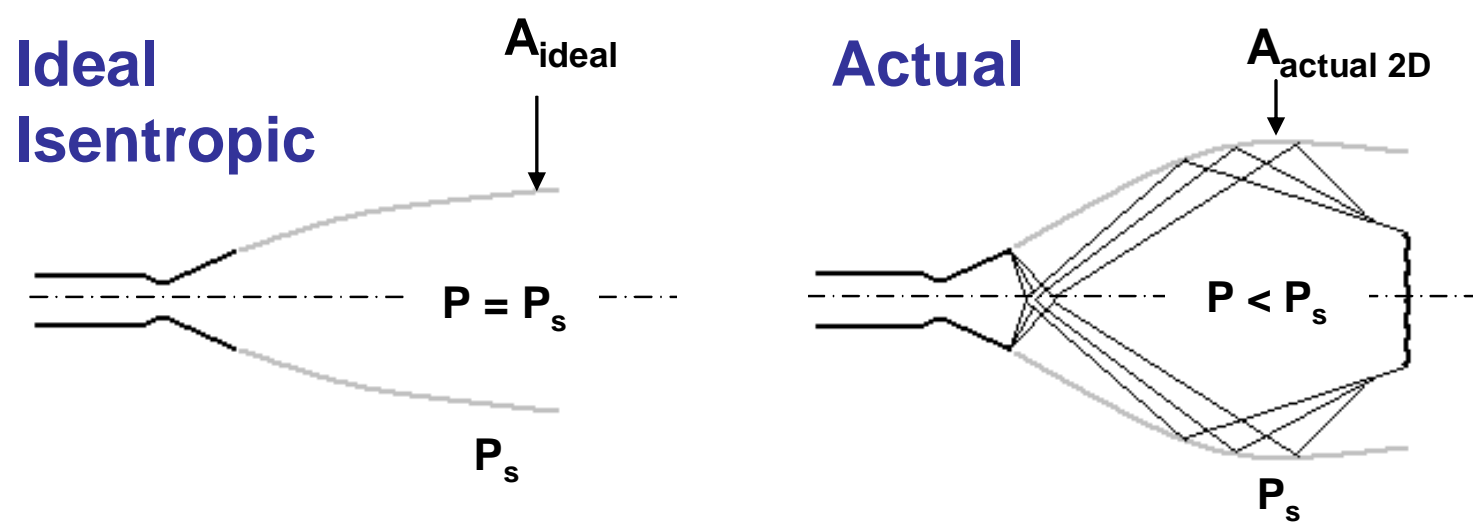

Figure 2-5 The variation between ideal and actual primary plume expansion ${ }^{4}$

The impact of the empirical growth factor on the primary plume can be seen in Figure 2-6.

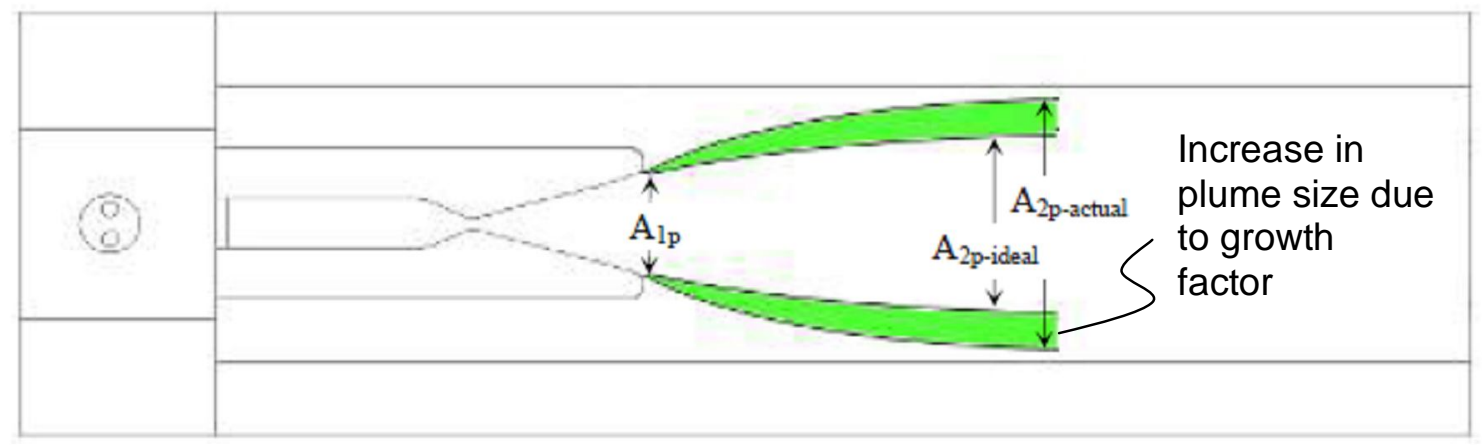

Figure 2-6 Growth of primary plume from ideal to actual size due to empirical correction factor ${ }^{10}$

\section{Methodology}

In order to estimate entrainment ratios and rapidly predict aerodynamic flow patterns, a computer code is written in the MATLAB language. The Cal Poly Supersonic Ejector (CPSE) computer simulation operates similar to the analyses presented by Fabri and Addy. First the primary stream geometry is developed. This task is performed using the Method of Characteristics (MOC). The flow properties in the secondary stream are determined from stagnation conditions and the shape of the primary plume using compressible isentropic relations. The primary stream uses the newly calculated pressure of the secondary stream to produce an updated set of values which approach the final solution. The primary and the secondary pressure distribution solutions iterate until the solution does not change considerably. Finally an entrainment ratio is calculated and the image of the converged simulation of the flow is displayed. 


\subsection{Assumptions}

Several assumptions are made in the analysis of the ejector flow properties. The geometry of the ejector is two-dimensional and planar. The upper and lower surfaces of the ejector must not converge, diverge or form any type of curve or oscillation. The flow must be steady and continuous. There are no considerations for unsteady or transient analysis including starting or stopping of the ejector. The flow must also be irrotational. The secondary stream is assumed quasi-one-dimensional. The primary plume is solved using the Method of Characteristics which requires irrotational flow. The viscous interaction between the primary plume and the secondary stream is considered negligible although it is commonly accepted that the viscous interaction can be a significant mechanism for mixing and energizing the secondary flow. The viscous interaction between the secondary stream and the duct wall is also neglected. The gases which make up the primary plume and the secondary stream are considered to be of the same temperature and chemical composition of air. Neither flow undergoes combustion at any stage of the ejector operation. It is also assumed that there are no strong shocks within the primary plume. The Method of Characteristics is able to handle weak compression shocks, however the sharp discontinuities formed by strong shocks and normal shocks are not able to be computed by the method. If a recirculation zone exists near the lip of the primary nozzle, this recirculation zone is assumed to be pressure matched to the secondary stream and the primary plume. The pressure within the recirculation zone may vary in the streamwise direction if required to form a continuous distribution with the surrounding flows, however the pressure in any recirculation zone is assumed constant in the transverse direction.

\subsection{Primary Plume Calculation Method}

The primary flow is calculated using the Method of Characteristics. The Method of Characteristics analysis is a more computationally expensive approximation of the primary plume than the one-dimensional and quasi-one-dimensional methods used by Fabri, Emanuel, Foster and Gist. However, the Method of Characteristics is able to provide an accurate primary plume boundary without the implementation of any correction factors or apriori knowledge. This boundary geometry is critical because it determines the properties in the secondary stream and the entrainment ratio. The Method 
of Characteristics has the additional benefit of producing a two-dimensional distribution of the properties within the primary plume including Mach number, pressure, temperature, density and any derivable attributes of the flow. The factors which influence the primary plume are the primary stagnation conditions, the nozzle geometry and the pressure distribution in the secondary flow. An initial guess of the secondary pressure distribution is required to start the Method of Characteristics. An intermediate iteration of the primary plume found using the Method of Characteristics is shown in Figure 3-1.

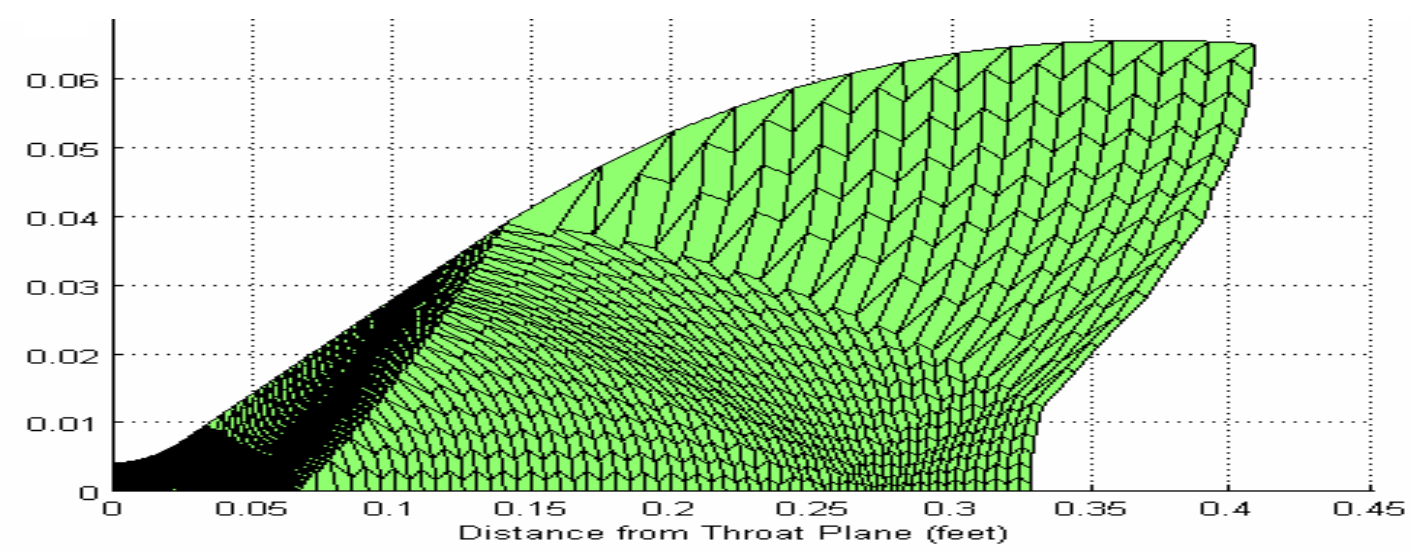

Figure 3-1 Definition of primary plume using Method of Characteristics The green area represents the primary plume, the area approximated by the Method of Characteristics. The black lines are characteristic lines along which the properties of the flow are transferred.

The algorithm used to describe the primary flow of the air-air ejector is adapted from Zucrow and Hoffman ${ }^{23,24}$. Zucrow and Hoffman present a FORTRAN algorithm of the Method of Characteristics, with which the background information provided is easily implemented into any computing language. For this study the algorithm is implemented in the MATLAB computing language.

The Method of Characteristics first establishes a set of initial values. The number of initial value points determines the baseline resolution of the MOC solution. Sixty initial value points were used for the CPSE simulation. From the initial values, the properties of the flow are computed at the plume's interior points, along the wall of the primary 
nozzle, and along a pressure matched free boundary beyond the nozzle. The Method of Characteristics Algorithm used is an adapted version of Riley's ${ }^{25}$ implementation of Zucrow and Hoffman's Method.

\subsubsection{Method of Characteristics Interior Point Calculation}

A point located in the interior of a supersonic plume is termed an interior point. A typical interior point is shown in Figure 3-2.

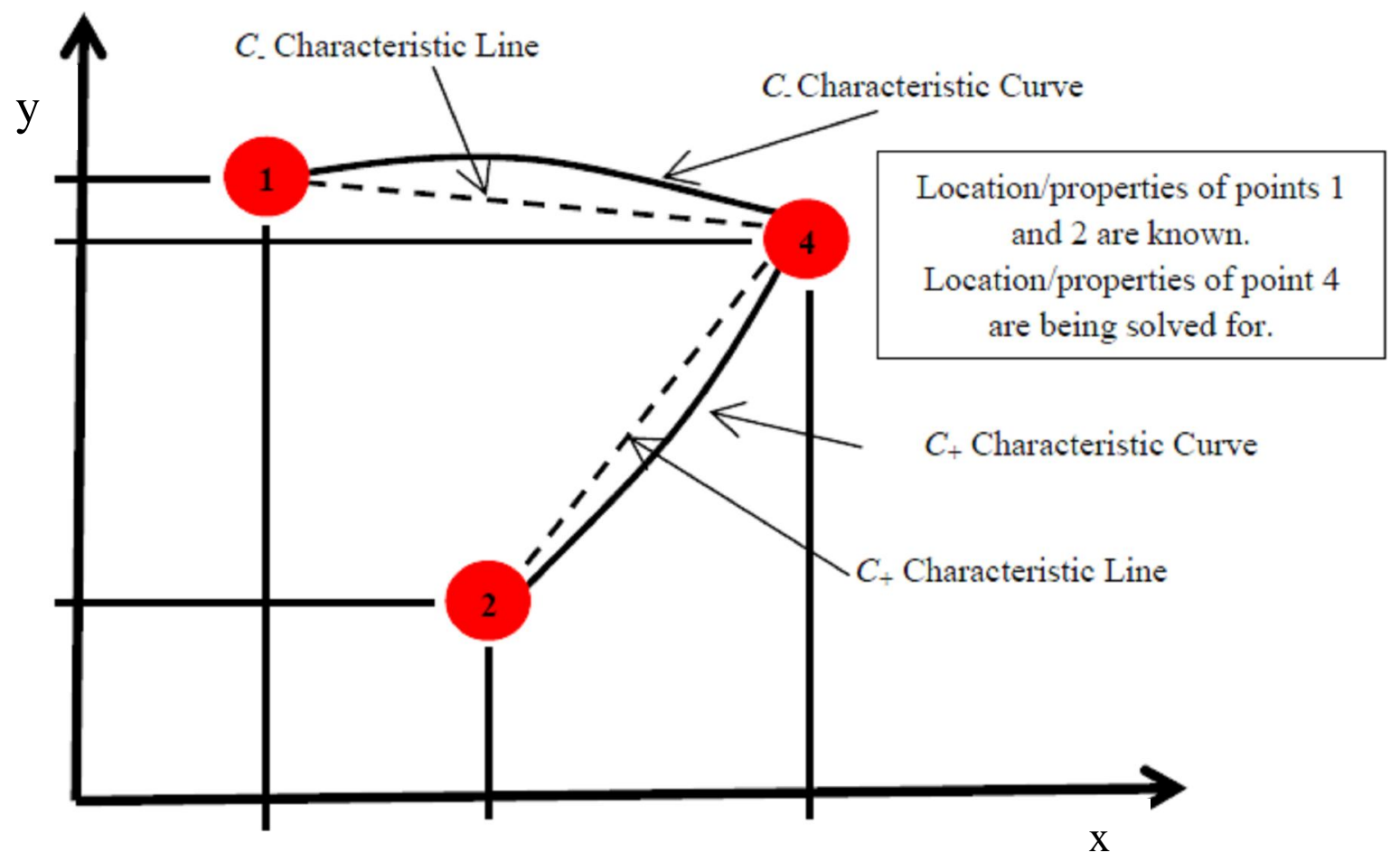

Figure 3-2 Method of Characteristics unit process for an interior point ${ }^{25}$

The interior point of interest, point 4 , is located at the intersection of the $C_{+}$and $C_{-}$characteristic lines from initial value points 1 and 2, which have known properties and locations. Equation 3.1 and Equation 3.2 are used to determine the transverse (y) and stream wise (x) location of point 4.

$$
\begin{aligned}
& y_{4}-\lambda_{+} x_{4}=y_{2}-\lambda_{+} x_{2} \\
& y_{4}-\lambda_{-} x_{4}=y_{1}-\lambda_{-} x_{1}
\end{aligned}
$$

Equation 3.2 
where

$$
\lambda_{ \pm}=\tan \left(\theta_{ \pm}+\alpha_{ \pm}\right)
$$

The angle of the velocity vector $(\theta)$ is measured counterclockwise from horizontal and $\alpha$ is the Mach angle. The + and - subscripts refer to the properties of the $C_{+}$and $C_{-}$ characteristic lines from initial value points 1 and 2. The values $u$ and $v$ are the stream wise and transverse velocity components of the primary flow.

$$
\theta_{ \pm}=\tan ^{-1}\left(\frac{v_{ \pm}}{u_{ \pm}}\right)
$$

Figure 3-3 shows how the angles $\theta$ and $\alpha$ are related to the characteristic lines and the streamline. The local slope of the streamline of the flow $(\theta)$ is used to determine the angle of the characteristic lines. The Mach angle $(\alpha)$ represents the region of influence of a point at a given Mach number. The triangle (or cone in three dimensions) formed by the lines tangent to the $C_{+}$and $C_{-}$characteristic lines is the region of influence of the point of known value, where the characteristics and streamline intersect. The known point does not directly influence the properties of any point outside of this region of influence. 


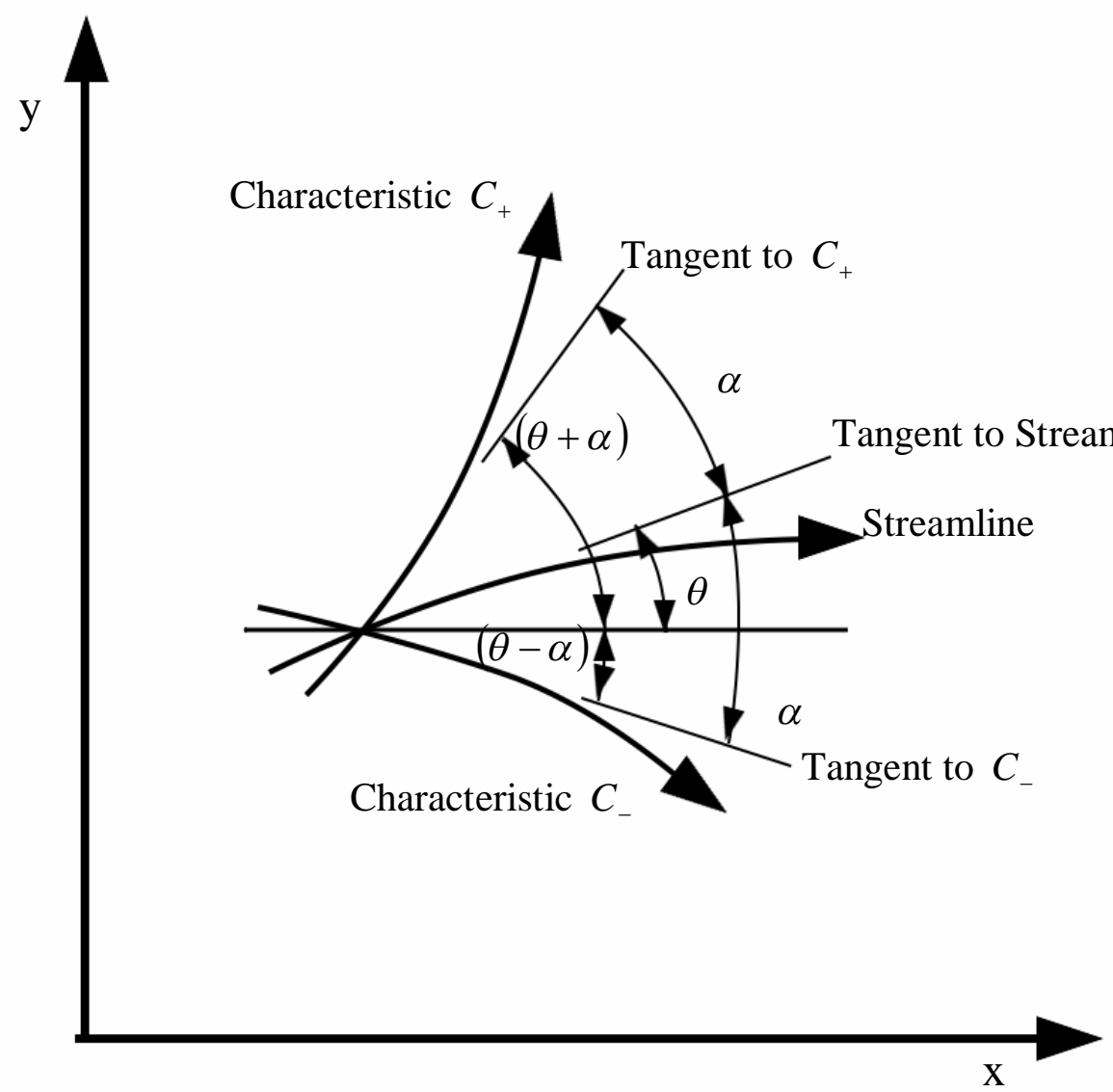

Figure 3-3 Definition of angles used in Method of Characteristics

The local velocity magnitude (V), speed of sound (a), Mach number (M) and Mach angle $(\alpha)$ can all be determined using the equations below.

$$
\begin{gathered}
V_{ \pm}=\sqrt{u_{ \pm}^{2}+v_{ \pm}^{2}} \\
a_{ \pm}=a\left(V_{ \pm}\right) \\
M_{ \pm}=\frac{V_{ \pm}}{a_{ \pm}} \\
\alpha_{ \pm}=\sin ^{-1}\left(\frac{1}{M_{ \pm}}\right)
\end{gathered}
$$

The compatibility Equation 3.3 is then implemented using the coefficients Q, R and T. These coefficients come from solving the governing differential equations. The full derivation can be found in Zucrow and Hoffman's Gas Dynamics. Only the equations required to implement the Method of Characteristics are shown. 


$$
Q_{ \pm} u_{4}+R_{ \pm} v_{4}=T_{ \pm}
$$

Equation 3.3

where

$$
\begin{gathered}
T_{+}=Q_{+} u_{2}+R_{+} v_{2} \\
T_{-}=Q_{-} u_{1}+R_{-} v_{1}
\end{gathered}
$$

with

$$
\begin{gathered}
Q_{ \pm}=\left(u_{ \pm}^{2}-a_{ \pm}^{2}\right) \\
R_{ \pm}=\left(2 u_{ \pm} v_{ \pm}-Q_{ \pm} \lambda_{ \pm}\right)
\end{gathered}
$$

A special case of an interior point is an axis of symmetry point. These occur along the centerline of the primary plume. Figure 3-4 shows a typical axis point, where point 4, the point of interest, is on the centerline.
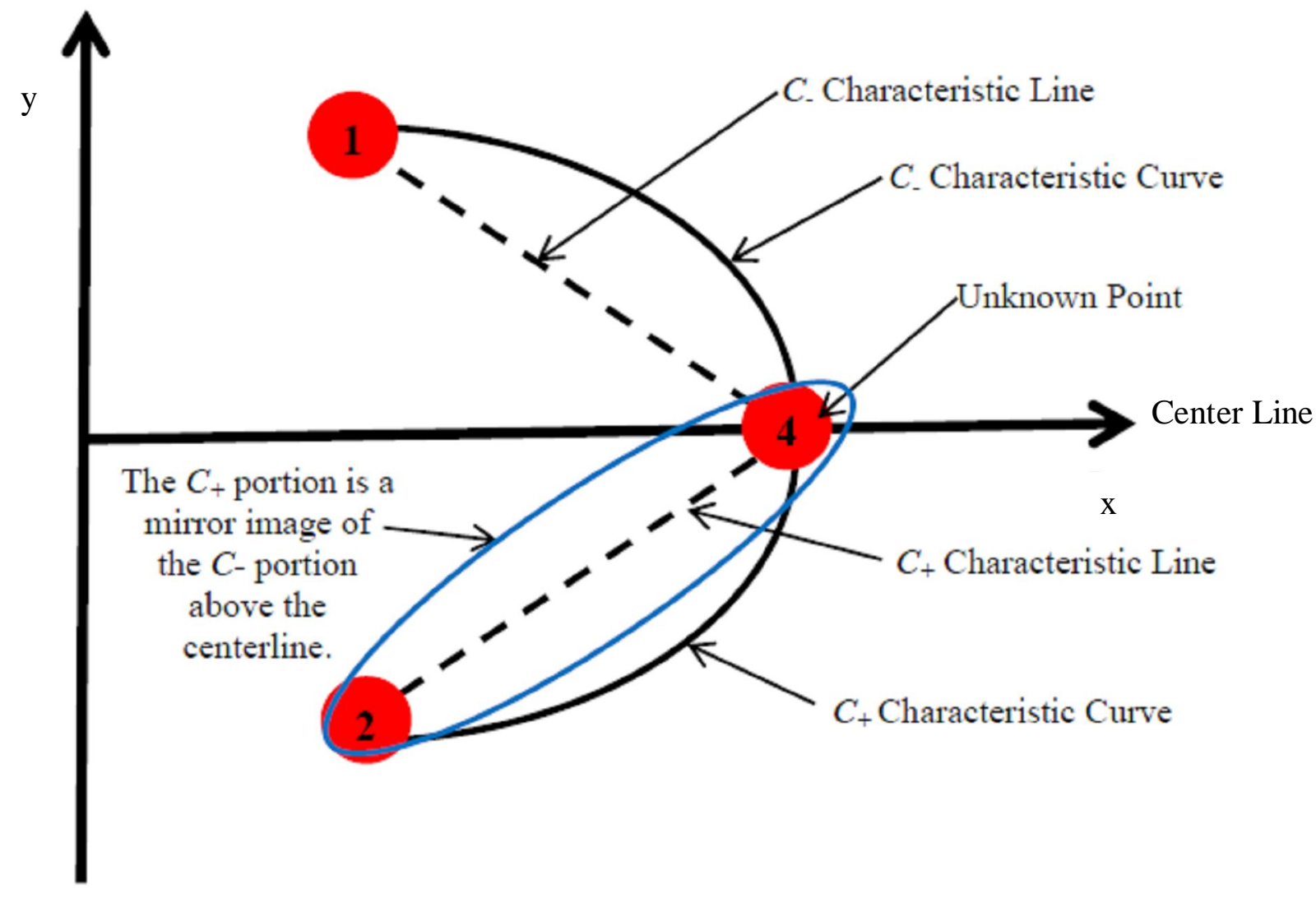

Figure 3-4 Method of Characteristics unit process for a symmetry point ${ }^{25}$ 
If point 1 is on a $C_{-}$characteristic line through point 4 , then it has a mirror image, point 2 , on the $C_{+}$characteristic. The symmetry point is then solved for using the interior process with the additional known values for transverse location (y), transverse velocity $(v)$ and flow angle $(\theta)$ in Equation 3.4. At this point, the location is on the centerline, the $\mathrm{x}$-axis, which has a transverse location of 0 . The direction of flow is found from point 1 and point 2 which have equal influence and are mirror images of each other about the $\mathrm{x}$-axis. Therefore, the transverse components of the velocity cancel and the flow is horizontal, resulting in a transverse velocity of 0 . With no transverse component of velocity, the streamline is along the centerline. The angle between the centerline and the flow is also 0 .

$$
y_{4}=v_{4}=\theta_{4}=0
$$

\subsubsection{Method of Characteristics Direct Wall Point Calculation}

A direct wall point occurs where the flow comes in contact with the wall of the primary nozzle. For this case the direction of the flow velocity must equal the local slope of the nozzle wall. The wall point 4 is defined where the $C_{+}$characteristic from known interior point 2 intersects the nozzle. A typical direct wall point is shown in Figure 3-5. 


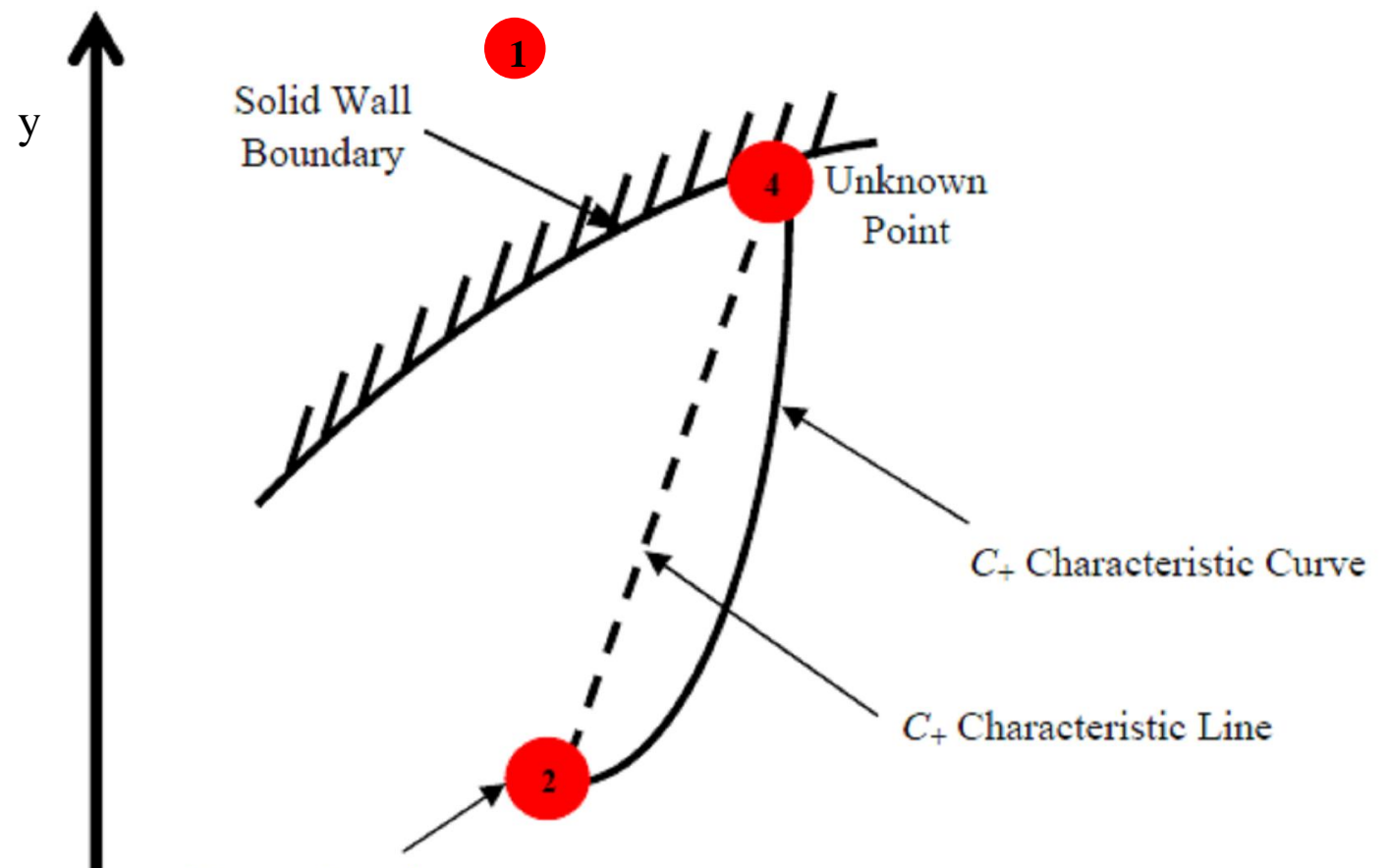

Known Interior

Point

Figure 3-5 Method of Characteristics unit process for a wall point ${ }^{25}$

Due to the $C_{-}$characteristic emanating from a point which does not physically exist in the flow field, point 1 , only one compatibility equation can be used to determine the location and properties of the wall point. However, the nozzle geometry offers the remaining required relationships of the transverse location $(y)$ of the point of interest in Equation 3.5 and the flow direction $(\theta)$ in relation to the slope of the nozzle $\left(\theta_{\text {nozzle }}\right)$ Equation 3.6. As shown in Figure 3-5 the point of interest with unknown properties lies on the wall of the nozzle. Therefore, the transverse location of the point of interest can be found once the stream wise location of the point is determined and input into the function defining the nozzle wall geometry. Not only must the location of the wall point conform to the nozzle geometry, the direction of flow of the wall points must also conform to the slope of the nozzle wall, making the nozzle wall a streamline.

$$
y_{4}=y_{\text {nozzle }}(x)
$$




$$
\frac{d y}{d x}=\tan \theta=\frac{v_{4}}{u_{4}}=\tan \theta_{\text {nozzle }}
$$

Using these equations as well as the familiar compatibility equations, Equation 3.1 and Equation 3.7, the location ( $\mathrm{x}$ and $\mathrm{y}$ ) and properties at point 4 are determinable using a similar process as the interior points. Recall Q, R and $\mathrm{T}$ are the coefficients found from solving the governing differential equations. The tangent of the slope of the $C_{+}$characteristic line emanating from point 2 is $\lambda_{+}$.

$$
\begin{gathered}
y_{4}-\lambda_{+} x_{4}=y_{2}-\lambda_{+} x_{2} \\
Q_{+} u_{4}+R_{+} v_{4}=T_{+}
\end{gathered}
$$

\subsubsection{Method of Characteristics Free Pressure Boundary Point Calculation}

The free pressure boundary point occurs on the boundary of the primary plume beyond the end of the primary nozzle. The fundamental characteristic of this condition is that the pressure on the boundary of the primary plume must match the pressure of the secondary flow. Figure 3-6 shows the unit process for a free pressure boundary point. 


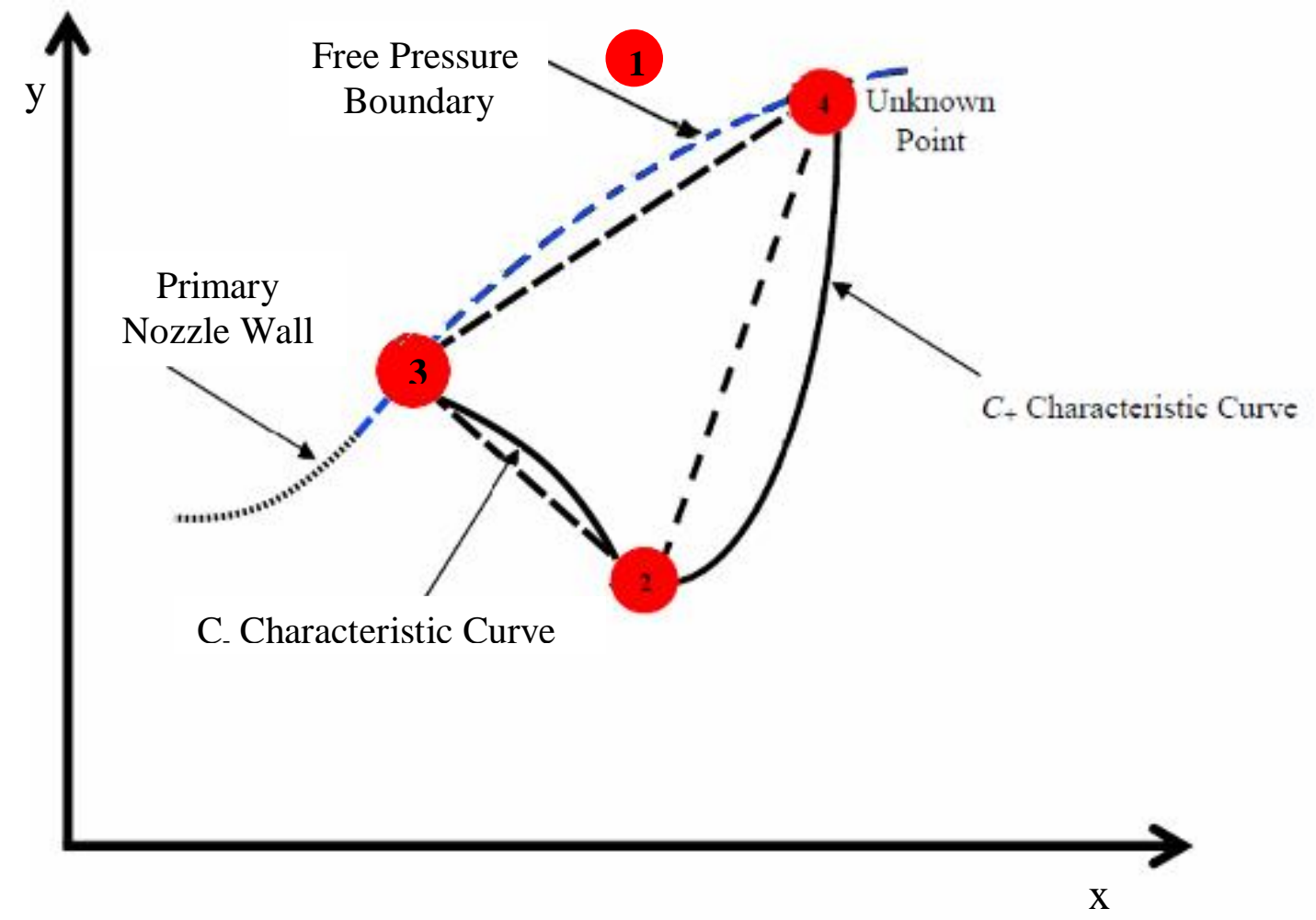

Figure 3-6 Method of Characteristics unit process for free pressure boundary point

The secondary pressure is known, or assumed. The CPSE simulation begins with a guess for the secondary pressure distribution. Once the primary plume has been solved for using the Method of Characteristics, an updated secondary pressure distribution is assumed. The total velocity magnitude $(\mathrm{V})$ and static pressure $(\mathrm{p})$ of the primary flow are related by isentropic flow properties. The velocity at point 4 , the location being solved for, is given by Equation 3.8 .

$$
V_{4}=\left(u_{4}^{2}+v_{4}^{2}\right)^{\frac{1}{2}}=f\left(p_{4}\right)=f\left(p_{s}\right)=\text { known }
$$

The local stream-wise and transverse velocities ( $u$ and v) are related to the coefficients of the finite difference equations, $\mathrm{Q}, \mathrm{R}$ and $\mathrm{T}$ by Equation 3.7.

$$
Q_{+} u_{4}+R_{+} v_{4}=T_{+}
$$


The simultaneous solutions of Equation 3.7 and Equation 3.8 yield formulations for the stream-wise and transverse velocities at the point of interest. These formulations are Equation 3.9 and Equation 3.10, respectively.

$$
\begin{gathered}
u_{4}=\frac{Q_{+} T_{+}-R_{+}\left[V_{4}^{2}\left(Q_{+}^{2}+R_{+}^{2}\right)-T_{+}^{2}\right]^{1 / 2}}{Q_{+}^{2}+R_{+}^{2}} \\
v_{4}=\left(V_{4}^{2}-u_{4}^{2}\right)^{1 / 2}
\end{gathered}
$$

The final relationship required for the solution of a free pressure boundary point, Equation 3.11, is the condition that the boundary of the primary plume is along a stream line. The direction of flow $\left(\frac{u}{v}\right)$, along line $3-4$, must be equal to the slope of the plume boundary, $\lambda_{0}$.

$$
\frac{d y}{d x}=\frac{v}{u}=\lambda_{0}
$$

The relationship between the components of the streamline along the free pressure boundary is shown graphically in Figure 3-7. 


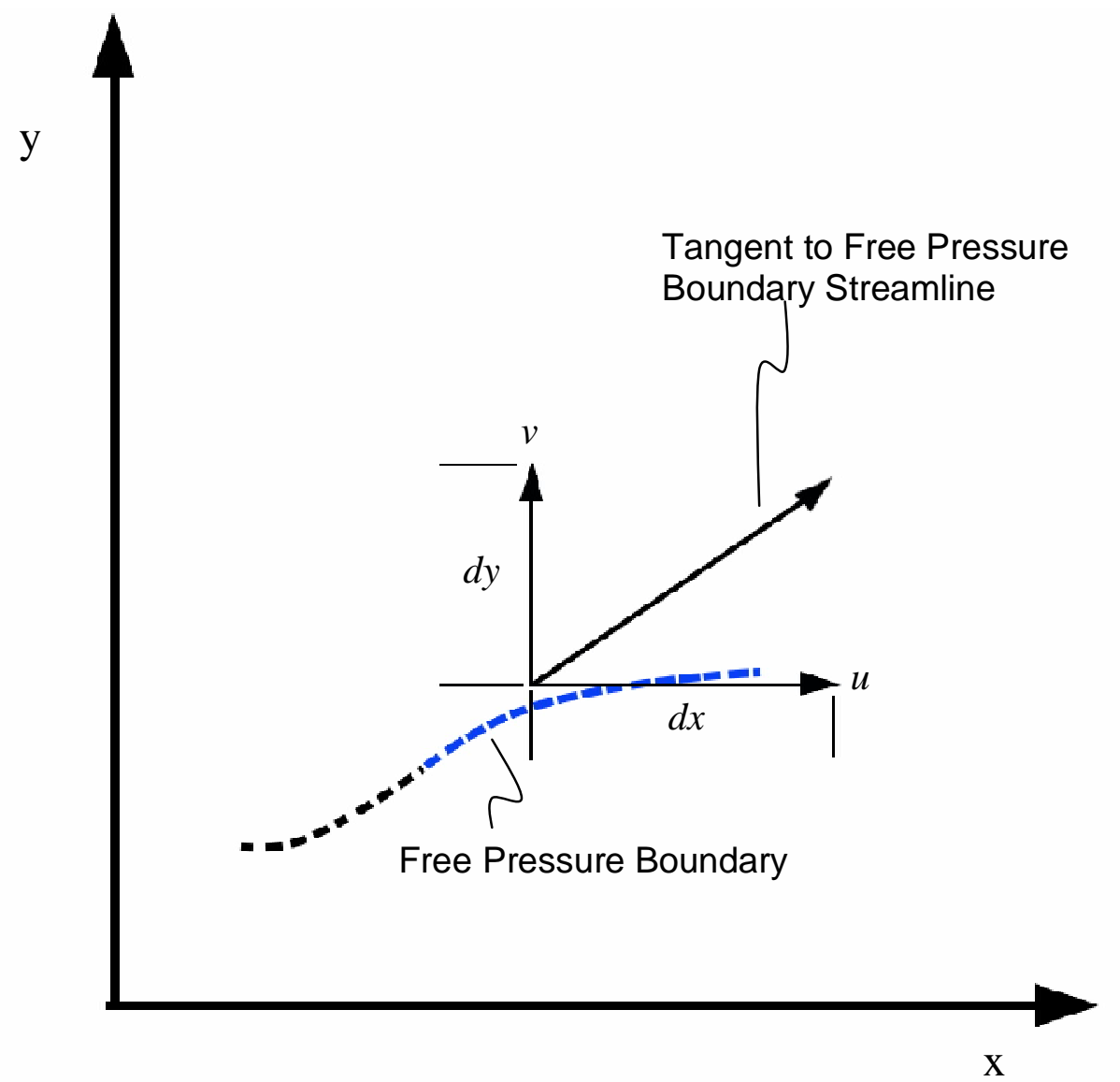

Figure 3-7 Components of the velocity vector along the free pressure boundary streamline

\subsection{Secondary Stream Calculation Method}

The secondary stream of the ejector is analyzed using one-dimensional isentropic relations. The secondary stream is divided into vertical slices. The isentropic relations are evaluated at each slice. The distribution of the "slices" is determined by the free pressure boundary points from the Method of Characteristics. This method of dividing the secondary stream to correspond closely with the free pressure boundary points reduces the amount of interpolating required by the Method of Characteristics algorithm while it matches the pressure distribution of the primary and secondary streams along the free pressure boundary.

The geometry of the primary plume determines the available flow area of the secondary stream. The secondary stagnation conditions are related to the allowable flow area to 
determine the properties within the flow. Figure 3-8 shows the Mach number distribution of the secondary flow using the isentropic area relations. The flow area of the secondary plume is bounded on top by the ejector duct wall. The primary nozzle wall and the primary plume serve as a lower bound of the secondary flow area. The secondary stream is discretized into tall cells separated by thin black lines. The properties within each cell are constant. The properties change only in the stream wise direction and are assumed constant in the transverse direction.

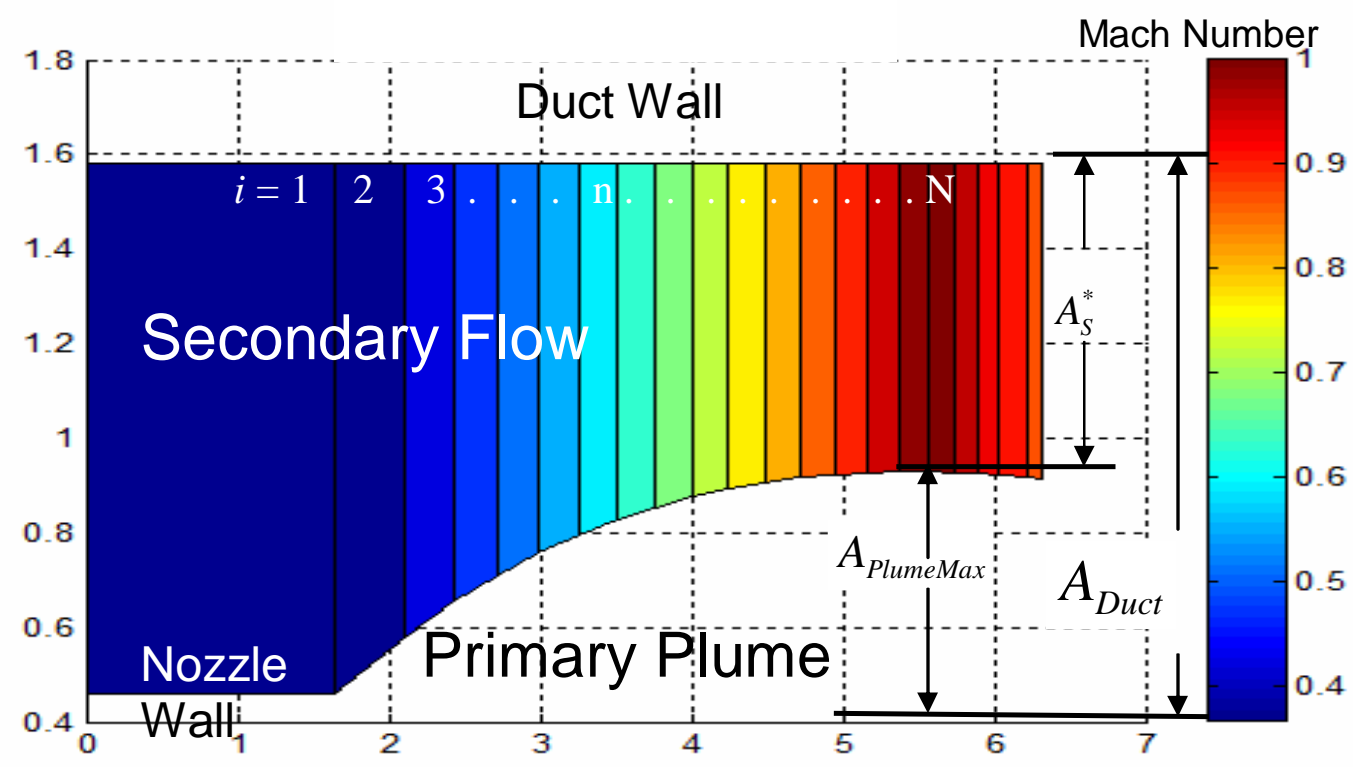

Figure 3-8 The Mach number of secondary flow using isentropic area relations.

\subsubsection{Mach number solution}

The Mach number distribution in the secondary flow is driven by the primary plume geometry. The minimum area of allowable secondary flow occurs where the difference between the area of the primary plume and the duct area $\left(A_{\text {Duct }}\right)$ is minimum. This occurs where the primary plume area is at a maximum $\left(A_{\text {PlumeMax }}\right)$. At the stream wise location of the minimum secondary flow area, the secondary flow area is set to the critical condition $\left(A_{S}^{*}\right)$. This is shown in Equation 3.12.

$$
A_{S}^{*}=A_{\text {Duct }}-A_{\text {PlumeMax }}
$$

Equation 3.12 
Using the known secondary area at each point in the streamwise direction $\left(A_{S i}\right)$ and Mach number of unity at the critical point, the Mach number of the secondary flow at each point in the streamwise direction $\left(M_{S i}\right)$ is found by solving Equation 3.13 iteratively ${ }^{23}$.

$$
\frac{1}{M_{S i}}\left(\frac{2}{\gamma+1}\right)\left(1+\frac{(\gamma-1) M_{S i}{ }^{2}}{2}\right)^{\left(\frac{\gamma+1}{2(\gamma-1)}\right)}=\frac{A_{S i}}{A_{S}^{*}}
$$

Equation 3.13

The subscript $i$ refers to the index used when discretizing the secondary stream into cells. The indexing, like the discretization it describes is derived from the free pressure boundary points found using the Method of Characteristics in the primary plume. However, the indexing scheme is used to describe the entire secondary stream, including the region before the mixing chamber. Therefore, the subscript 1 would be reserved for the cell which begins at the secondary inlet and ends at the exit of the primary nozzle. The properties in cell 1 are constant because the properties are determined using inviscid calculations of the isentropic relations with no heat addition. Since there is no area change, the Mach number and subsequent properties are constant in this area. The designation of the cells continues sequentially with the secondary cell indicated by subscript 2. When an arbitrary cell is being referred to the subscript $i$ is used. Equation 3.13 uses the $i$ subscript notation to indicate that the Mach number of any cell which can be found using the available area of secondary flow into that same cell. From the area of the cell face (the left hand side of any cell of interest in Figure 3-8) the Mach number is found and assumed to be constant within the cell. The properties within the secondary flow are solved for using the newly found Mach number distribution.

\subsubsection{Isentropic values}

The secondary stagnation properties and the Mach number distribution determine the properties in the secondary flow. The secondary stagnation temperature $\left(T_{0 S}\right)$, pressure $\left(P_{0 S}\right)$ and density $\left(\rho_{0 S}\right)$ are known from the conditions from which the ejector is entraining flow. Typically these properties are based on ambient conditions at the 
entrance of the ejector. The secondary conditions may not be equal to the ambient or exit conditions, for instance, if the secondary flow is entrained from a plenum.

Within each cell of the discretized secondary stream, the local static temperature $\left(T_{S i}\right)$, pressure $\left(p_{S i}\right)$, and density $\left(\rho_{S i}\right)$ is found using the Mach number of the cell of interest of the secondary flow $\left(M_{S i}\right)$ and the isentropic Mach number relations shown in Equation 3.14, Equation 3.15 and Equation $3.16^{23}$. The ratio of specific heats $(\gamma)$ for the fluid being entrained into the secondary flow is also required for these calculations.

$$
\begin{gathered}
T_{S i}=\frac{T_{0 S}}{1+\frac{\gamma-1}{2} M_{S i}^{2}} \\
p_{S i}=\frac{P_{0 S}}{\left(1+\frac{\gamma-1}{2} M_{S i}^{2}\right)^{\frac{\gamma}{\gamma-1}}} \\
\rho_{S i}=\frac{\rho_{0 S}}{\left(1+\frac{\gamma-1}{2} M_{S i}^{2}\right)^{\frac{\gamma}{\gamma-1}}}
\end{gathered}
$$

\subsection{Iteration Scheme}

As previously stated, the primary and secondary streams drive the properties within the other. The primary plume geometry is dependent on the pressure distribution of the secondary stream. The pressure distribution of the secondary stream is dependant on the geometry of the primary plume. In order to start the calculation process, an initial guess of the pressure distribution of the secondary stream is made. This initial guess is then 
refined through iterations of the primary plume geometry feeding into the secondary stream properties and the secondary stream properties shaping the primary plume geometry. Left unaltered, this iteration scheme is highly unstable and slow to converge on a solution.

\subsubsection{Relaxation Factors}

A relaxation factor is implemented to reduce fluctuations in the iteration scheme. The relaxation factor works by incrementally changing the secondary pressure distribution guess in the direction of the final solution. The secondary pressure distribution $\left(p_{S}\right)$ to be used for the Method of Characteristics in the next iteration is found as a weighted combination of the newly found static pressure distribution $\left(p_{\text {SNew }}\right)$ and the pressure distribution from the previous iteration $\left(p_{\text {sold }}\right)$. The weighting is determined by the relaxation factor $(\mathrm{k})$ and shown in Equation 3.17. The value of the relaxation factor can vary between 0 and 1 . A fully relaxed case with relaxation factor of 1 will have no update and never converge. Using a relaxation factor of 0 provides no relaxation factor. The results jump violently from one iteration to the next and may become unstable or never converge. A relaxation factor between 0.75 and 0.85 provides a fairly stable convergence patterns and run times on the order of 90 seconds. Iteration count and run times increase near the limits of aerodynamic flow patterns. For instance the iteration count and run time of a case which borders on a blocked aerodynamic flow pattern and a Fabri choke condition may take several minutes because the values of the intermediate iterations jump between maximum secondary Mach numbers of 1 for the Fabri choke condition and secondary Mach numbers of 0 for the blocked case.

$$
p_{S}=(k) p_{\text {SOld }}+(1-k) p_{\text {SNew }}
$$

Equation 3.17

\subsection{Entrainment Ratio Calculation}

The entrainment ratio of the ejector is the primary issue being investigated in this study. Entrainment ratios and similar measurements have been discussed by Fabri, Addy, Emanuel, Foster, Gist and others as a standard for ejector performance. The experimental entrainment ratios found by Gist provide a benchmark with which to evaluate the 
accuracy of the CPSE simulation. The entrainment ratio $(\beta)$ is the ratio of the mass flow rate of the secondary stream $\left(\dot{m}_{s}\right)$ relative to the mass flow rate of the primary stream $\left(\dot{m}_{p}\right)$. The entrainment ratio shown in Equation 3.18 is a common measure of ejector efficiency.

$$
\beta=\frac{\dot{m}_{S}}{\dot{m}_{p}}
$$

Equation 3.18

As Equation 3.18 indicates, the mass flow rates of the secondary and primary streams must be solved for in order to calculate the entrainment ratio. However, because the ejector under investigation is two-dimensional and planar, the height of the ejector is constant; therefore the height is not accounted for in the simulation. In reality, varying the height (h) of the ejector would cause changes in performance due to fictional losses on the ejector surfaces because a different portion of the flow would be affected by the boundary layer. However, for a two-dimensional planar ejector with an inviscid analysis, this height term will cancel out of the entrainment ratio equation without changing the result; as is apparent in Equation 3.19.

$$
\beta=\frac{\dot{m}_{S} / h}{\dot{m}_{P} / h}
$$

Although the terminology is not completely accurate, the values of mass flow rate divided by height will still be referred to as mass flow rates.

The primary mass flow rate is found at the primary nozzle throat using the nozzle geometry and the stagnation conditions. The critical area of the primary nozzle throat $\left(A_{P}^{*}\right)$ is actually the width of the throat. As previously stated, the height of the ejector is not considered. The chamber pressure of the primary stream $\left(P_{0 P}\right)$, the chamber temperature of the primary stream $\left(T_{0 P}\right)$, the ratio of specific heats $(\gamma)$ and gas constant $(R)$ for the fluid of the primary plume are also required. Since the primary and secondary flows are assumed to be similar in the CPSE simulation and experiments, the 
gas constant and ratio of specific heats of the primary stream fluid are considered to be the same as those of the secondary stream fluid. It is evident in Equation 3.20 that the mass flow rate of the primary plume is not affected by any of the secondary flow properties.

$$
\dot{m}_{p}=\left(\frac{A_{P}^{*} \gamma P_{0 P}}{\sqrt{\gamma R T_{0 P}}}\right)\left(\frac{2}{\gamma+1}\right)^{\left(\frac{\gamma+1}{2[\gamma-1]}\right)}
$$

Equation 3.20

The secondary mass flow rate is calculated at the point where the secondary stream achieves a sonic condition. This occurs at the point of minimum secondary flow area. This either occurs in the mixing chamber due to the expansion of the primary plume as in the Fabri choke case, or it occurs in the entrance of the duct before being exposed to the primary plume as in the saturated flow condition. The secondary mass flow rate $\left(\dot{m}_{S}\right)$ is dependent on the geometry of the primary plume and the critical density $\left(\rho_{S}^{*}\right)$ and velocity $\left(V_{S}^{*}\right)$ within the flow as shown in Equation 3.21. The critical static pressure $\left(p_{S}^{*}\right)$, density, temperature $\left(T_{S}^{*}\right)$ and velocity were found using, Equation 3.22, Equation 3.23, Equation 3.24 and Equation 3.25 and the stagnation conditions of the secondary flow. The ratio of specific heats and the gas constant of the secondary flow fluid are also required.

$$
\begin{gathered}
\dot{m}_{S}=\rho_{S}^{*} A_{S}^{*} V_{S}^{*} \\
p_{S}^{*}=P_{0 S}\left(\frac{2}{\gamma+1}\right)^{\left(\frac{\gamma}{\gamma-1}\right)}
\end{gathered}
$$

Equation 3.22 


$$
\begin{gathered}
\rho_{S}^{*}=\frac{\rho_{0 S}}{\left[1+\frac{(\gamma-1)}{2}\right]^{\left(\frac{\gamma}{\gamma-1}\right)}} \\
T_{S}^{*}=\frac{T_{0 S}}{\left(1+\left[\frac{\gamma-1}{2}\right]\right)} \\
V_{S}^{*}=\sqrt{\gamma R T_{S}^{*}}
\end{gathered}
$$

Equation 3.24

Equation 3.25

This analysis is used for the saturated case and the Fabri choke condition. The exception to this process is the trivial blocked case, there is no location of sonic condition in the secondary flow and the mass flow rate is zero.

\section{Results of the CPSE Simulation}

Unlike the one-dimensional, Fabri and Addy methods, this analysis is not concerned with the flow properties beyond the point of minimum secondary flow area. The analysis is only concerned with the region from the primary nozzle throat until the primary plume achieves maximum expansion. Within these parameters, three cases are possible. The three possible aerodynamic flow patterns are the saturated case, the supersonic case, also termed Fabri choke, and the blocked case. The secondary stream becomes choked in the saturated and Fabri choke conditions. The blocked case is unique in that the secondary stream is not choked. The secondary flow velocity is zero in the blocked case.

All of the simulations are run using the geometry of the existing CPSE experimental test rig. The primary and secondary streams are both composed of air with similar stagnation temperatures. The height of the ejector is 0.75 inches. The primary nozzle has a throat width of 0.1 inches and an exit width of 1.0 inches. The expansion ratio of the primary is nozzle 10 . The primary nozzle has a divergence angle of $15.35^{\circ}$. The lip of the nozzle 
base is 0.25 inches thick. Each of the two secondary inlet ducts has a width of 0.625 inches. Within the mixing chamber the duct walls diverge at an angle of $3^{\circ}$. This $3^{\circ}$ divergence was implemented as a requirement for tests in which combustion occurs in the mixing chamber. This divergence is not taken into account in the CPSE simulation and does not exist in updated revisions of the CPSE test apparatus. A dimensioned top view of the CPSE test apparatus is shown in Figure 4-1.

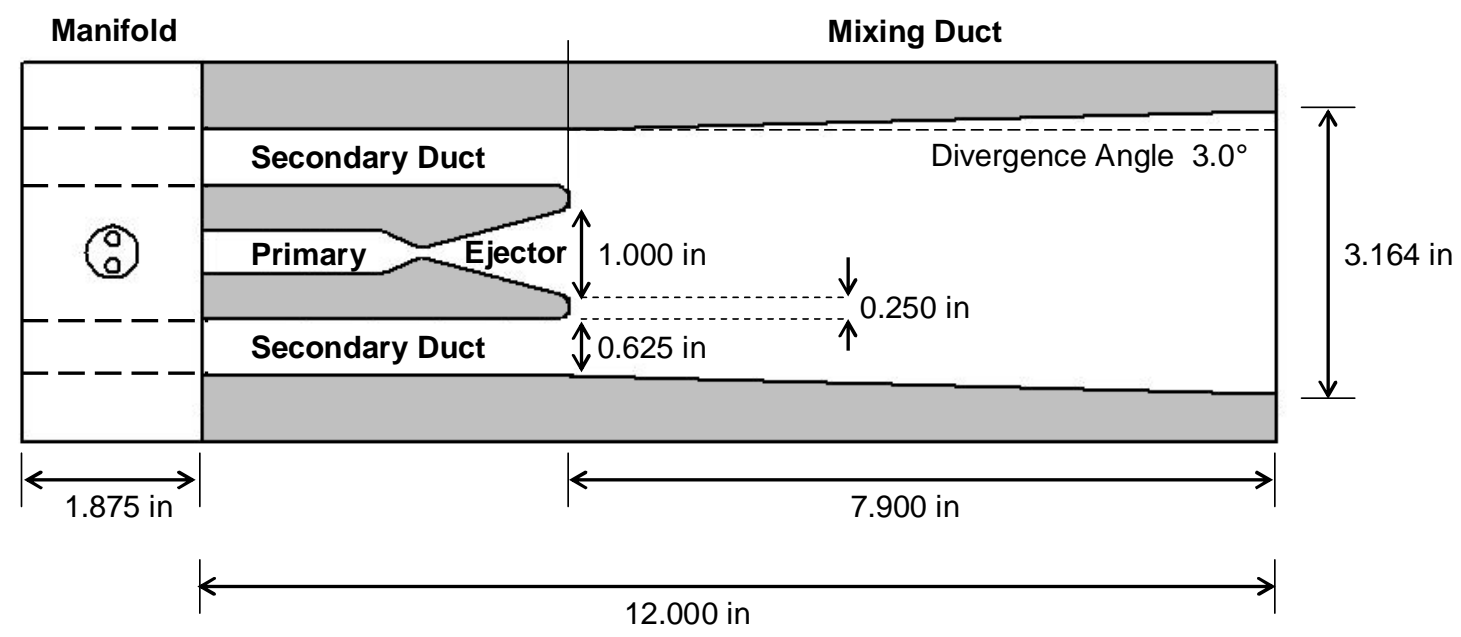

Figure 4-1 Dimensioned top view of the CPSE test apparatus ${ }^{4}$

\subsection{Saturated Supersonic Flow Pattern}

The lowest stagnation pressure ratios achievable with supersonic ejectors yield the saturated aerodynamic flow pattern. The saturated case is characterized by secondary flow achieving a critical Mach number before coming into contact with the primary plume. This condition is caused by the relatively high secondary stagnation pressure dominating the primary plume shape. For the CPSE configuration the saturated condition will occur with pressure ratios below 73; although, this value is dependant on numerous factors, especially geometry.

Figure 4-2 shows a CPSE simulation of the Mach number distribution of a saturated flow pattern with a primary to secondary stagnation pressure ratio of 70 . The large secondary stream flow area and stagnation pressure allow the optimal amount of fluid to be 
entrained by the supersonic primary plume. For this pressure ratio an entrainment ratio of 0.18 is achieved.

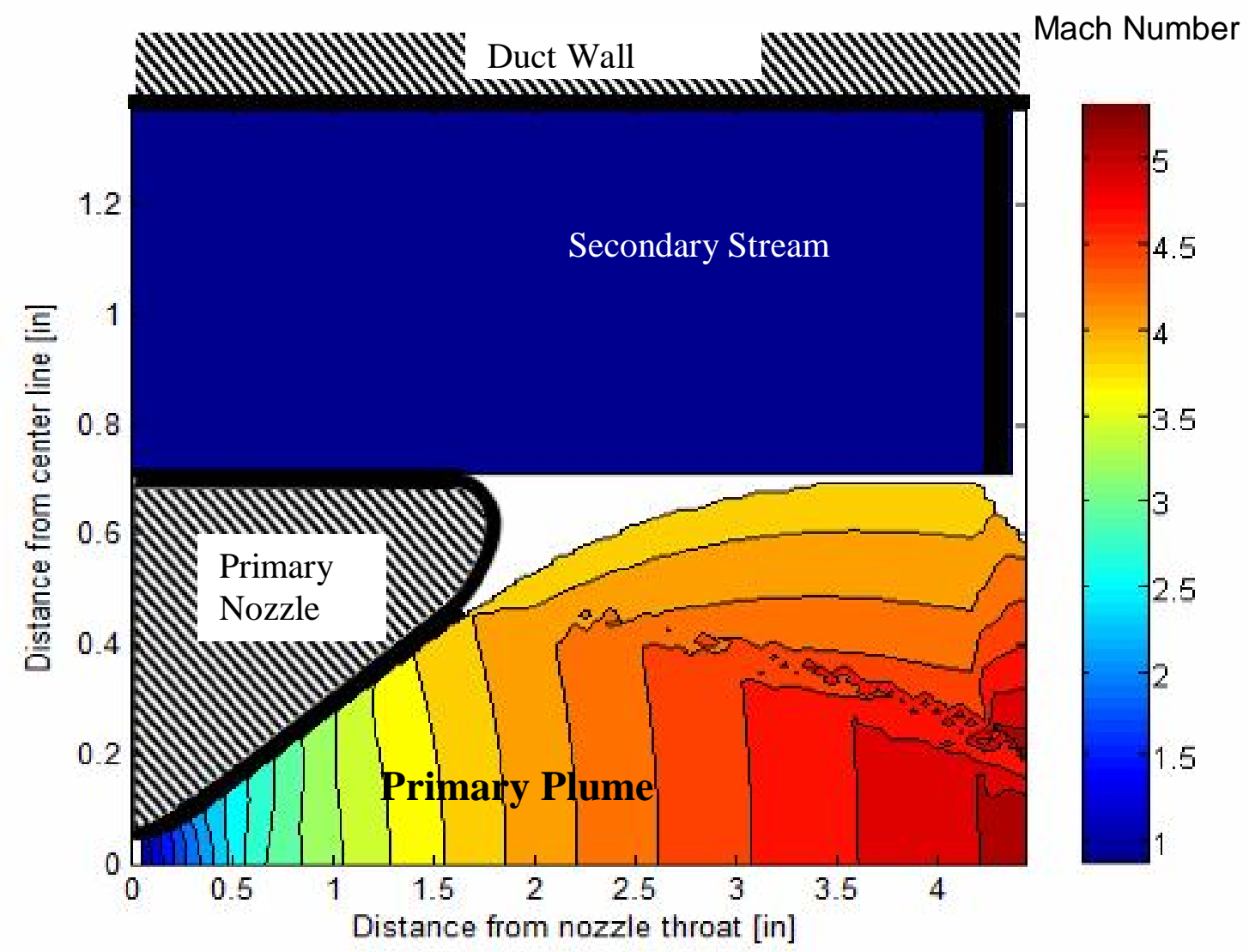

Figure 4-2 CPSE simulation of the Mach number distribution of a saturated flow pattern

Figure 4-2 shows a truncated view of the Mach number distribution within the flow field. First, only the top half of the flow field is shown because the flow is symmetric about the centerline of the primary nozzle (the $\mathrm{x}$ axis). Also, once the primary plume has achieved the maximum area, the Method of Characteristics calculations halt and the values are fed forward for secondary stream calculations. Once the primary plume has achieved the maximum area, the entrainment ratio can be determined. The properties of the flow field beyond this point are not of interest to this investigation because finding the entrainment ratio and the location where the secondary flow becomes sonic is the goal of the calculation. It is important to note that the axes of the plot have also been skewed to 
provide a more detailed view of the flow phenomena occuring. The transverse direction is enlarged compared to the streamwise direction.

The secondary flow is bounded on the upper surface by the duct wall. The primary nozzle and the primary plume are the lower bound of the secondary plume. The secondary stream achieves a critical Mach number in the inlet of the secondary stream before coming into contact with the primary plume. The Mach number of the secondary stream remains constant throughout the flow field.

The Mach number distribution in Figure 4-2 and the static pressure distribution in Figure 4-3 show many of the same trends. Both plots show that a large recirculation zone exists in the area bounded by the primary nozzle, the primary plume and the secondary stream. This recirculation zone is assumed to be pressure matched to the secondary stream and the boundary of the primary plume. Since the Mach number in the secondary stream is constant, the secondary pressure distribution is also constant. This requires the recirculation zone to have a constant pressure as well. Both physically and in the CPSE simulation, the recirculation zone is caused by the thickness of the nozzle lip. The nozzle lip cannot be brought to a sharp point due to structural requirements. This recirculation zone allows the primary plume boundary to gradually turn until it becomes parallel to the secondary stream flow direction. 


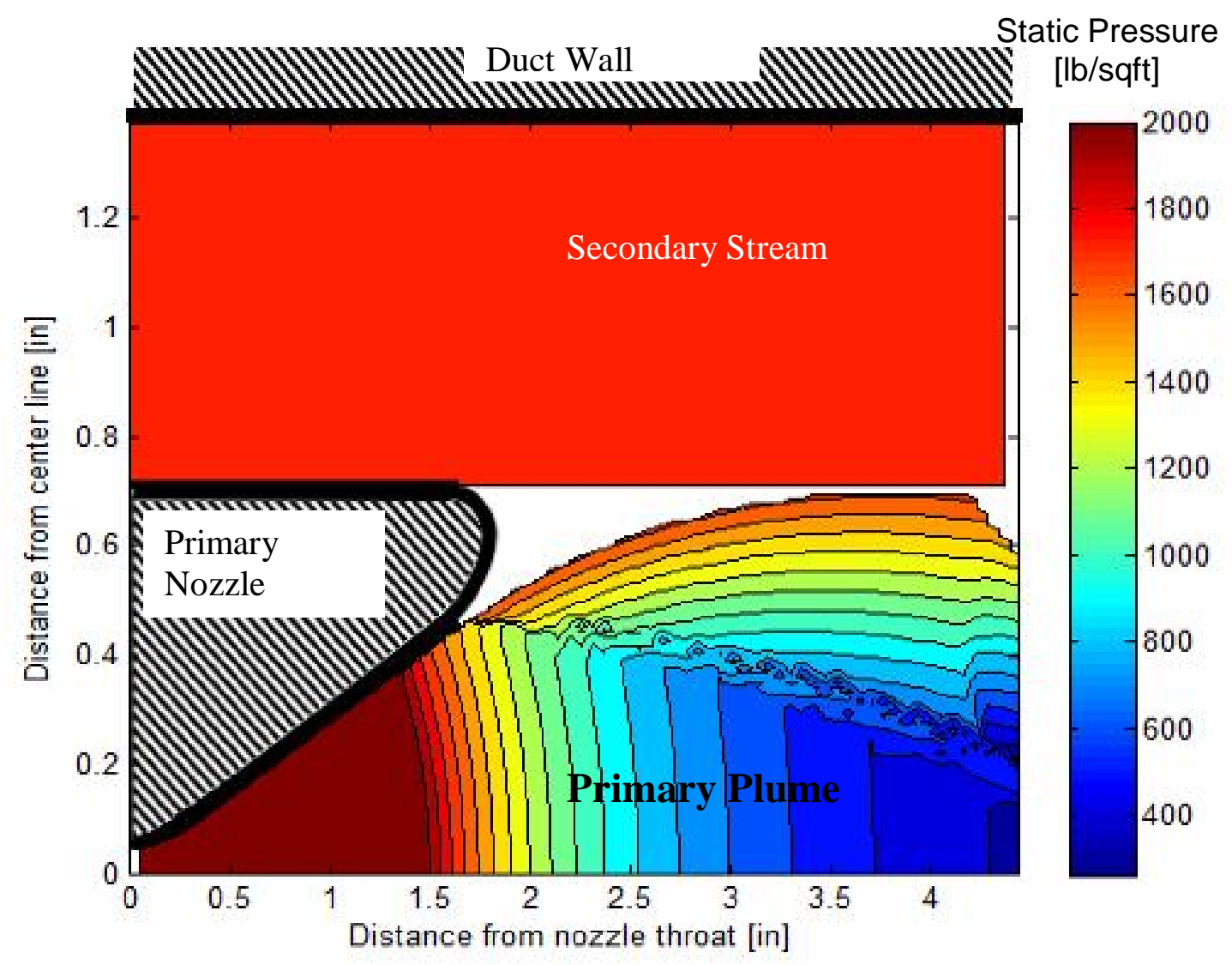

Figure 4-3 CPSE simulation of the static pressure distribution of a saturated flow pattern

Even with the gradual turning of the primary plume boundary facilitated by the recirculation zone, a compression shock emanates from the nozzle lip due to the interaction with the secondary stream. The secondary flow in the saturated case has relatively high energy compared to the other aerodynamic flow patterns. The oblique shock traces the peaks of the contour lines from the nozzle lip to the centerline of the flow. This shock was also detected in experimental testing. This shock emanating from the nozzle lip occurs to some extent in all the aerodynamic flow patterns. However, the shock is the strongest in this case, determined by the angle it travels from the nozzle lip and the effect on the Mach number and pressure beyond the shock. The strength of the shock causes a discontinuity in the properties of the flow which is not handled well by the MOC scheme. The MOC scheme smears this change of properties though a finite area. 
Some nodes downstream of the shock appear to be small regions of increased Mach number or decreased pressure. This phenomenon is numerical and not physical.

For this saturated condition, the secondary flow becomes choked before coming into contact with the primary plume. The primary plume is allowed to expand in the mixing chamber due to the recirculation zone caused by the thickness of the primary nozzle lip. The primary plume achieves maximum expansion 4 inches beyond the throat of the primary nozzle for the given geometry and a pressure ratio of 70 .

\subsection{Fabri Choke Supersonic Flow Pattern}

The supersonic Fabri choke condition is characterized by the secondary flow achieving a critical Mach number due to expansion of the primary plume. The secondary flow chokes in the mixing chamber where the primary plume achieves its maximum area. This aerodynamic flow pattern occurs when the primary to secondary stagnation pressure ratio is too high to result in the saturated case and too low to block the secondary flow. Typical pressure ratios for this condition with the CPSE configuration range between 73 and 230, this range of pressure ratios will vary with numerous factors, especially ejector geometry.

Figure 4-4 shows a CPSE simulation of the Mach number distribution of a Fabri choke supersonic flow pattern. This case was run with a primary to secondary stagnation pressure ratio of 125 . The ratio of secondary to primary mass flow rates is 0.073 . 


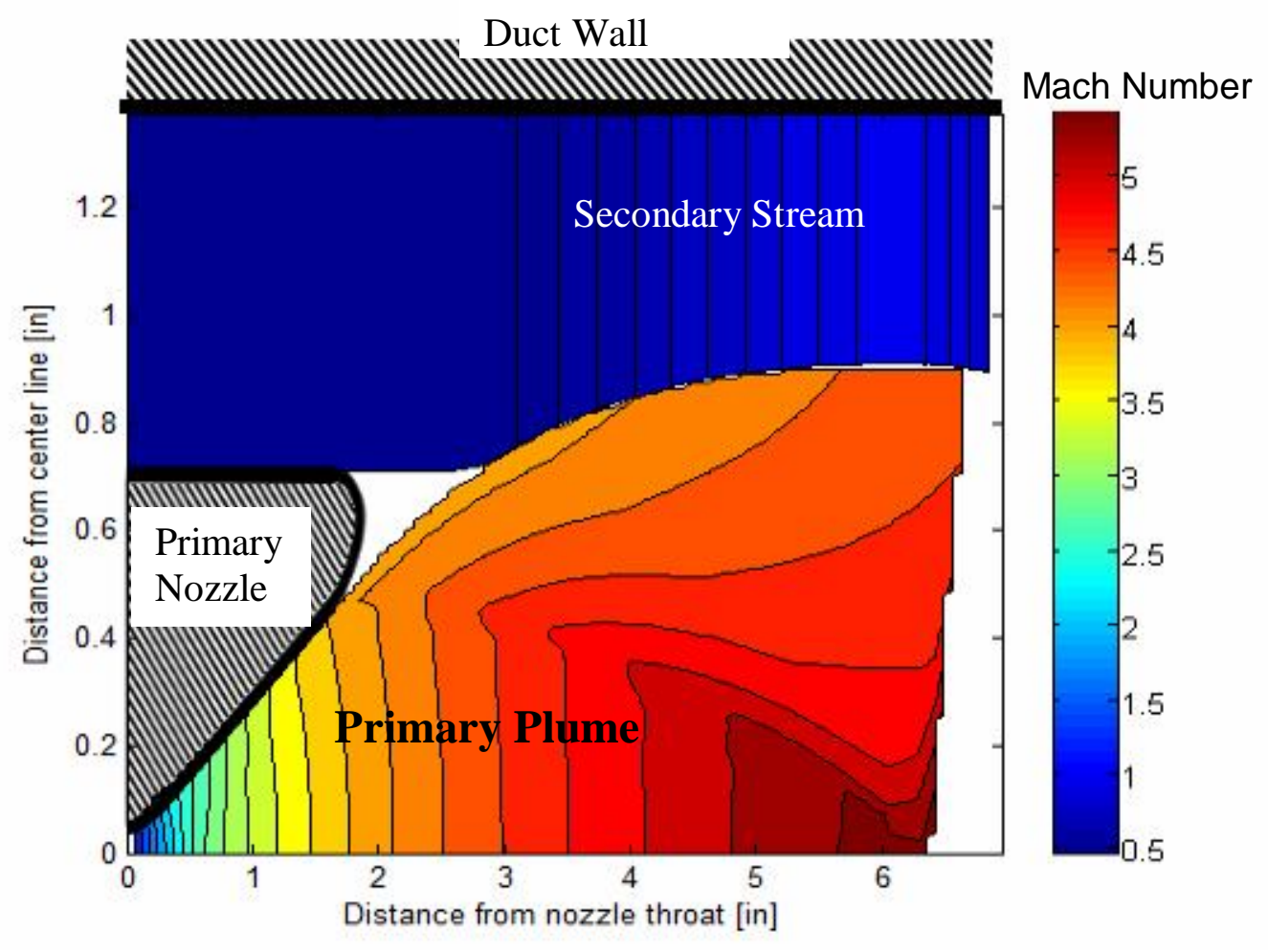

Figure 4-4 CPSE simulation of the Mach number distribution of a Fabri choke supersonic flow pattern

The incoming secondary stream is subsonic with a Mach number near 0.5. The secondary flow is accelerated by the primary plume until it reaches the critical velocity at the point of minimum secondary flow area. The primary to secondary stagnation pressure ratio is higher than the saturated case, enabling the primary plume to expand, reducing the secondary flow area.

Both Figure 4-4 and the static pressure distribution in Figure 4-5 show that the Fabri choke supersonic case also shows the compression shock in the primary plume emanating from the lip of the primary nozzle. The shock hinders the acceleration of the primary plume as the plume expands into the secondary flow area.

As in the saturated case, there is a recirculation zone beyond the lip of the nozzle. The recirculation zone occurring in Fabri choke conditions is much smaller than that of the 
saturated conditions which have a higher secondary flow rate. Similar to the secondary stream, the pressure in the recirculation zone varies in the streamwise direction, but not this transverse direction. This is intuitive for this analysis because the recirculation zone must match the pressure of the secondary stream and the primary plume, which are pressure matched as a requirement of the Method of Characteristics. In reality, the recirculation zone and the secondary stream will have two-dimensional variations in properties. Although physically the pressure within the recirculation zone may vary in the transverse direction, the static pressure on the boundary of the recirculation zone must match the secondary and primary streams.

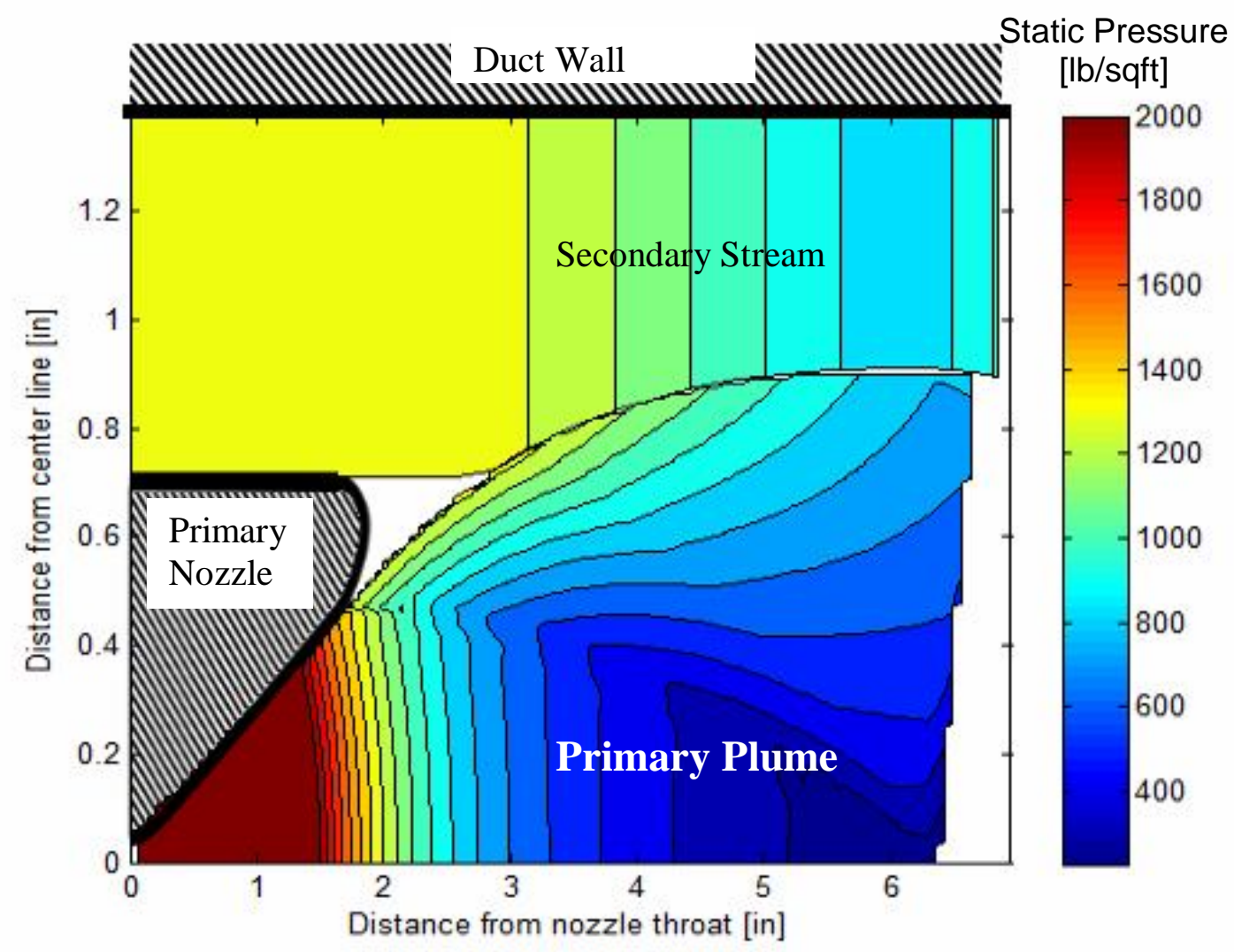

Figure 4-5 CPSE simulation of the static pressure distribution of a Fabri choke supersonic flow pattern

The Fabri choke condition is unique in that the Mach number in the secondary flow varies. In the saturated case, the Mach number is unity throughout the secondary flow. There is no net flow in the blocked case causing the Mach number to be 0 and the static 
pressure to be the stagnation pressure. However, the plume in the Fabri choke case must match the continuously variable pressure distribution of the secondary stream. The continuous pressure matching of the streams is displayed in Figure 4-5.

For this trial, the primary plume achieves maximum expansion nearly 6 inches beyond the exit plane of the primary nozzle in the stream wise direction. This location of maximum plume expansion is significantly further down stream than that of the saturated condition. Note the change in scale when comparing the saturated case to the Fabri choke cases.

\subsection{Blocked Flow Pattern}

The blocked case is characterized by a primary plume which comes in contact with the duct walls, blocking off the secondary flow. The primary to secondary stagnation pressure ratio must be very high to achieve a blocked condition. Although it is heavily dependent on geometry, for the CPSE geometry, the blocked case will generally occur above pressure ratios near 230. Figure 4-6 is a CPSE simulation of the Mach number distribution of a blocked flow pattern. The primary to secondary stagnation pressure ratio for this case is 250 . There is no secondary flow entrained. 


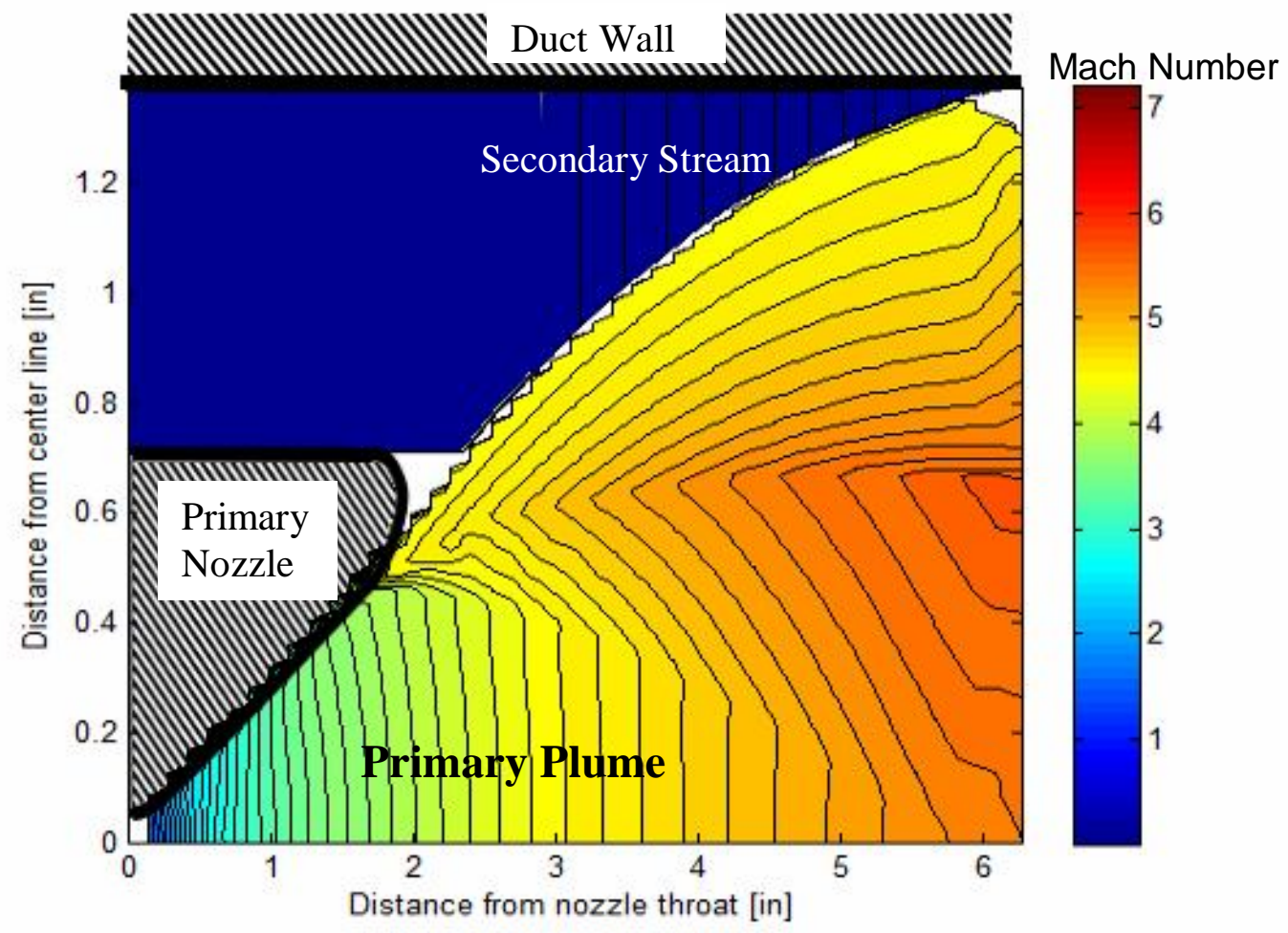

Figure 4-6 CPSE simulation of the Mach number distribution of a blocked flow pattern

The large primary to secondary stagnation pressure ratio facilitates rapid expansion of the primary plume. The primary plume expands all the way to the duct wall. The secondary flow is completely blocked. The incoming secondary flow rate is zero. Although there may be some recirculation in this area, the net mass flow of the secondary flow is zero. The low secondary stagnation pressure results in the least amount of flow turning of the primary plume. This reduced influence on the primary plume induces the weakest compression off the primary nozzle lip. The high primary chamber pressure also results in the highest primary Mach number.

Figure 4-7 shows the static pressure distribution of the blocked flow pattern. Similar to the saturated case, the static pressure in the secondary stream is constant. For this condition, the secondary static pressure is the stagnation pressure from which the air is being entrained. This constant pressure in the secondary stream means that the primary plume boundary is also constant in pressure. 


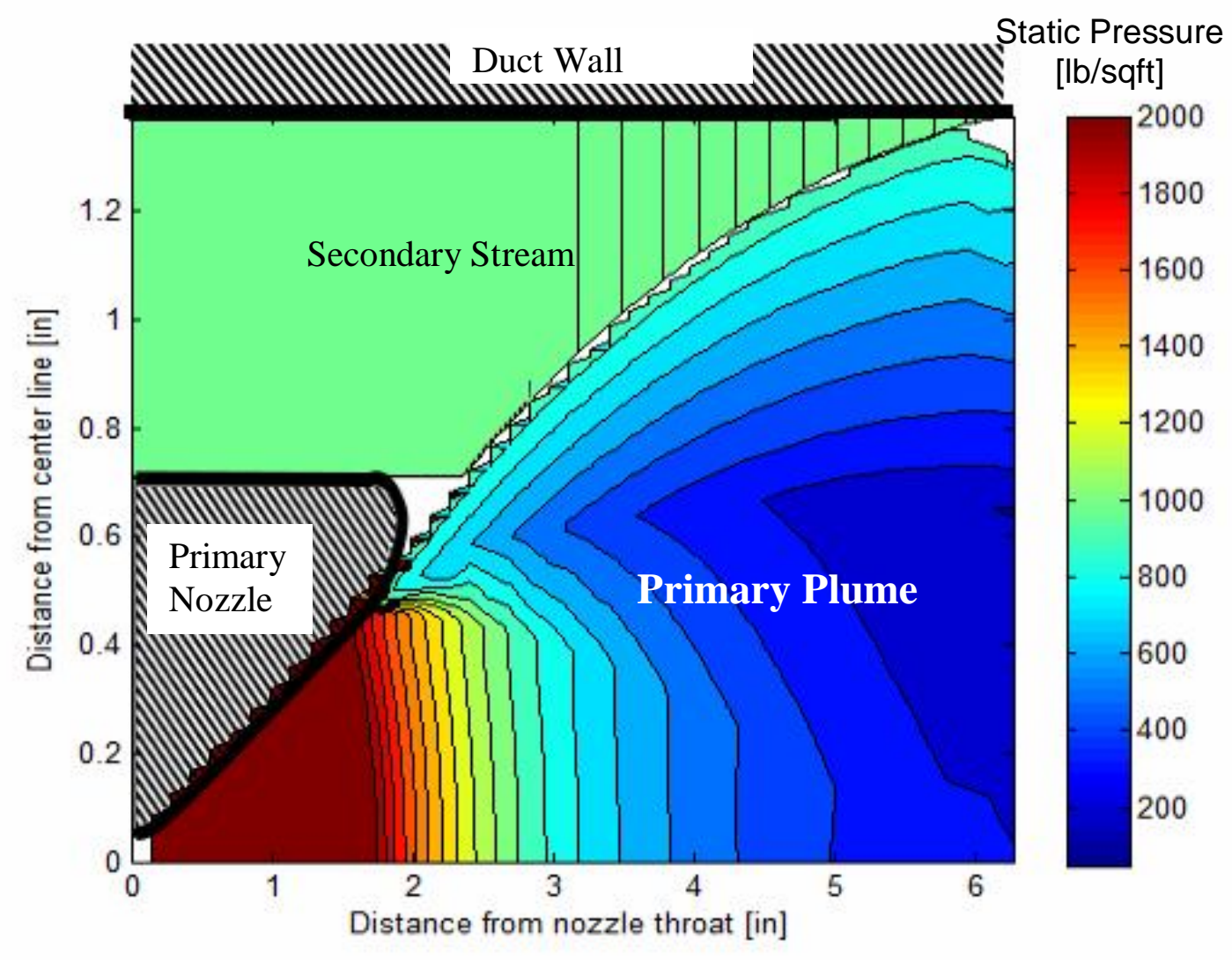

Figure 4-7 CPSE simulation of the static pressure distribution of a blocked flow pattern

The primary plume intersects the duct wall approximately 6.5 inches from the primary nozzle in the streamwise direction. This streamwise location of maximum primary plume expansion is comparable to that of the Fabri choke case. However, this maximum plume expansion location is definitively further downstream than both the Fabri choke and the saturated aerodynamic flow patterns. However, it cannot be assumed that the maximum expansion of the blocked case will always be the largest. At a pressure ratio of 262, the primary plume intersects the duct wall less than 5.5 inches downstream of the primary nozzle throat. Once the aerodynamic flow pattern reaches a blocked case, increasing the pressure ratio will decrease the distance where the primary plume achieves maximum expansion. This phenomenon is easily explained. The primary plume expands at a steeper angle when the secondary stagnation pressure is lower. This more rapid expansion of the primary plume causes the plume to intersect the duct wall at a location 
further upstream than cases of lower pressure ratios where the secondary stream hinders the expansion of the primary plume.

\section{Comparisons to Previous Work}

In order to evaluate the quality of the results from the CPSE computer simulation, previous work is used as a standard. The experimental and theoretical investigations of Gist provide a database with which to measure accuracy. The one-dimensional methods used by Gist and Foster to approximate the flow field properties within the ejector do not provide high fidelity illustration of the flow interactions. However, Gist and Foster both

made attempts to characterize the geometry of the primary plume. Flow visualization and entrainment ratio observations from these previous studies are used to validate the accuracy of the CPSE simulation.

The most readily verifiable hypothesis posed on the geometry of the flow field was Foster's proposal that the streamwise location of maximum primary plume expansion is unchanged by pressure ratio. The results from the CPSE simulation clearly show that as pressure ratio is increased, the location of maximum plume expansion moves downstream until the blocked flow pattern exists. Once the blocked flow pattern occurs, the point where the primary stream reaches maximum expansion begins to move back upstream. The CPSE simulation produced data which adds fidelity to the hypothesis of Foster. The flow visualization results interpreted by Foster and Gist produce this result within the accuracy capability of the flow visualization technique. Figure 5-1 and Figure 5-2 show the visualizations of Gist and Foster in which the streamwise direction measurement starts at the primary nozzle lip while the CPSE simulation takes the primary nozzle throat to be the starting point of the measurement. 


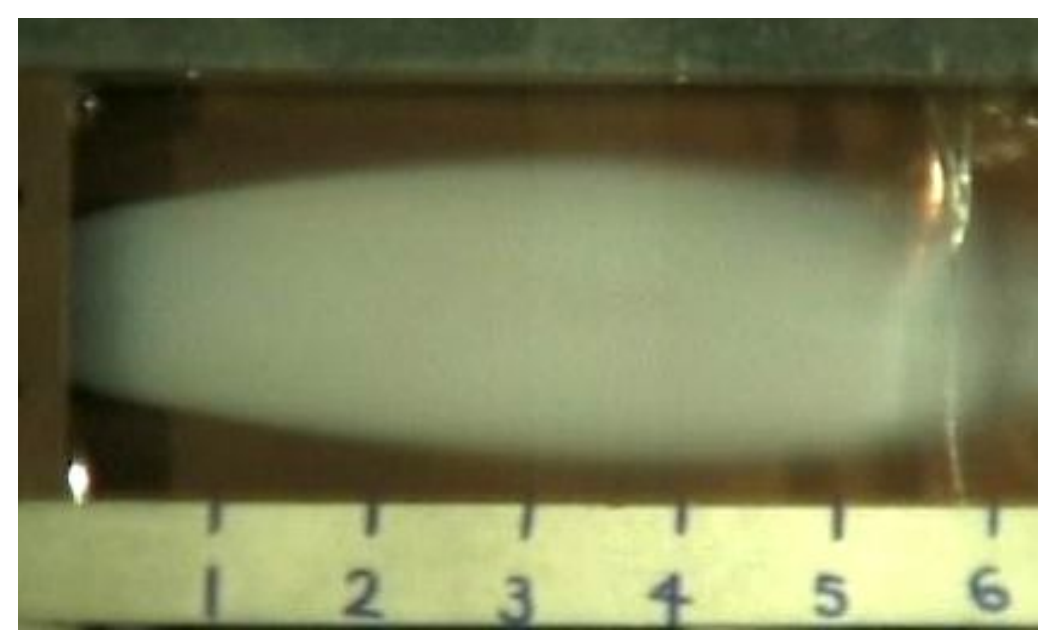

Figure 5-1 Nitrogen condensation plume in the mixing chamber at a pressure ratio of $95^{4}$

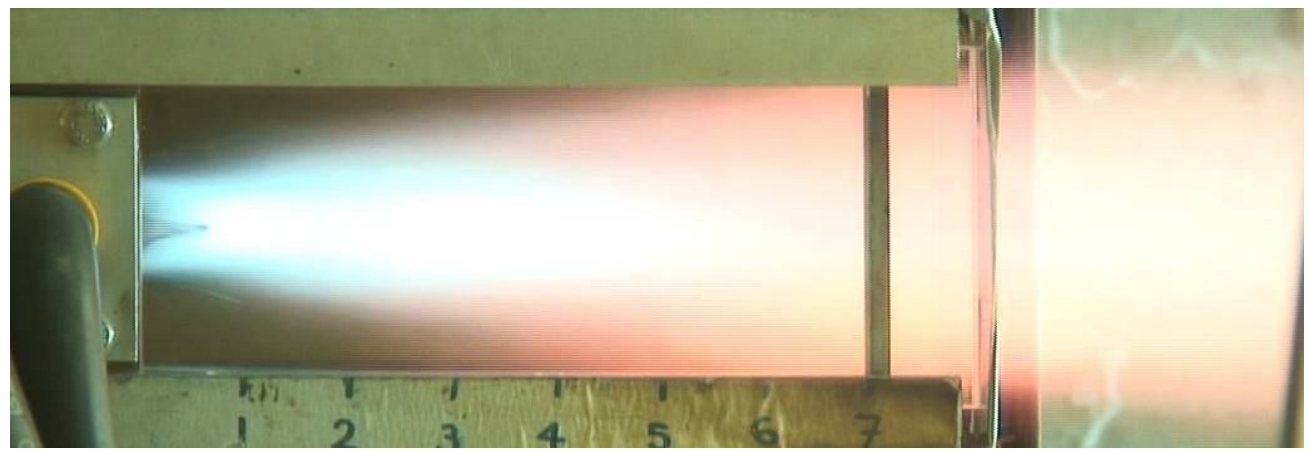

Figure 5-2 Methane and Oxygen thruster fired at a pressure ratio of $\mathbf{2 0}^{\mathbf{1 0}}$

Gist estimated the shock structure within the primary plume based on the condensation patterns in the photographic flow visualization of his experiments. Figure 5-3 shows the condensation shock structure recorded by Gist as well as Gist's superimposed shock structure. The image displays the expansion and compression cycle emanating from the lip of the nozzle. The onset of this shock cycle is also present in all aerodynamic flow patterns calculated by the CPSE simulation and shows the expected increase in size as the pressure ratio increases. 


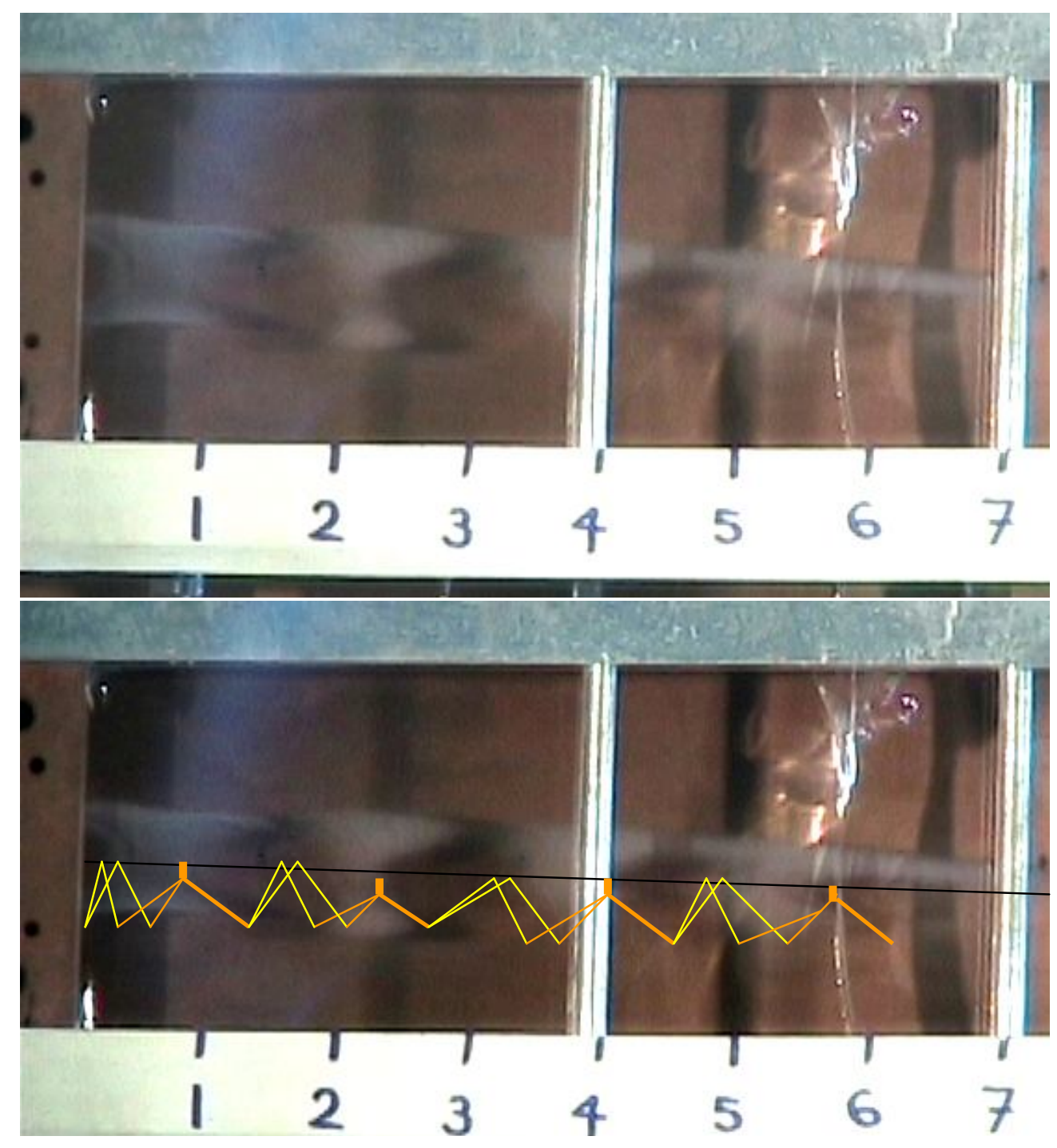

Figure 5-3 Video frame and superimposed shock structure from cold flow tests ${ }^{4}$

In addition to showing the start of expansion compression cycles, the CPSE simulation results also match CFD studies which have been conducted on the recirculation zones near the nozzle lip of supersonic ejectors ${ }^{30}$. Figure 5-4 shows the work of Purdue researchers Gujarathi, Li, Anderson and Sankaran. The low secondary flow rate represents a case of higher pressure ratio than the case of high secondary flow rate. The observations of the simulation results section above indicate that as the secondary flow decreased from saturated to Fabri choke to blocked cases, the size of the recirculation zone shrinks. Figure 5-4 reaffirms that larger secondary flow rates result in larger recirculation zones. 

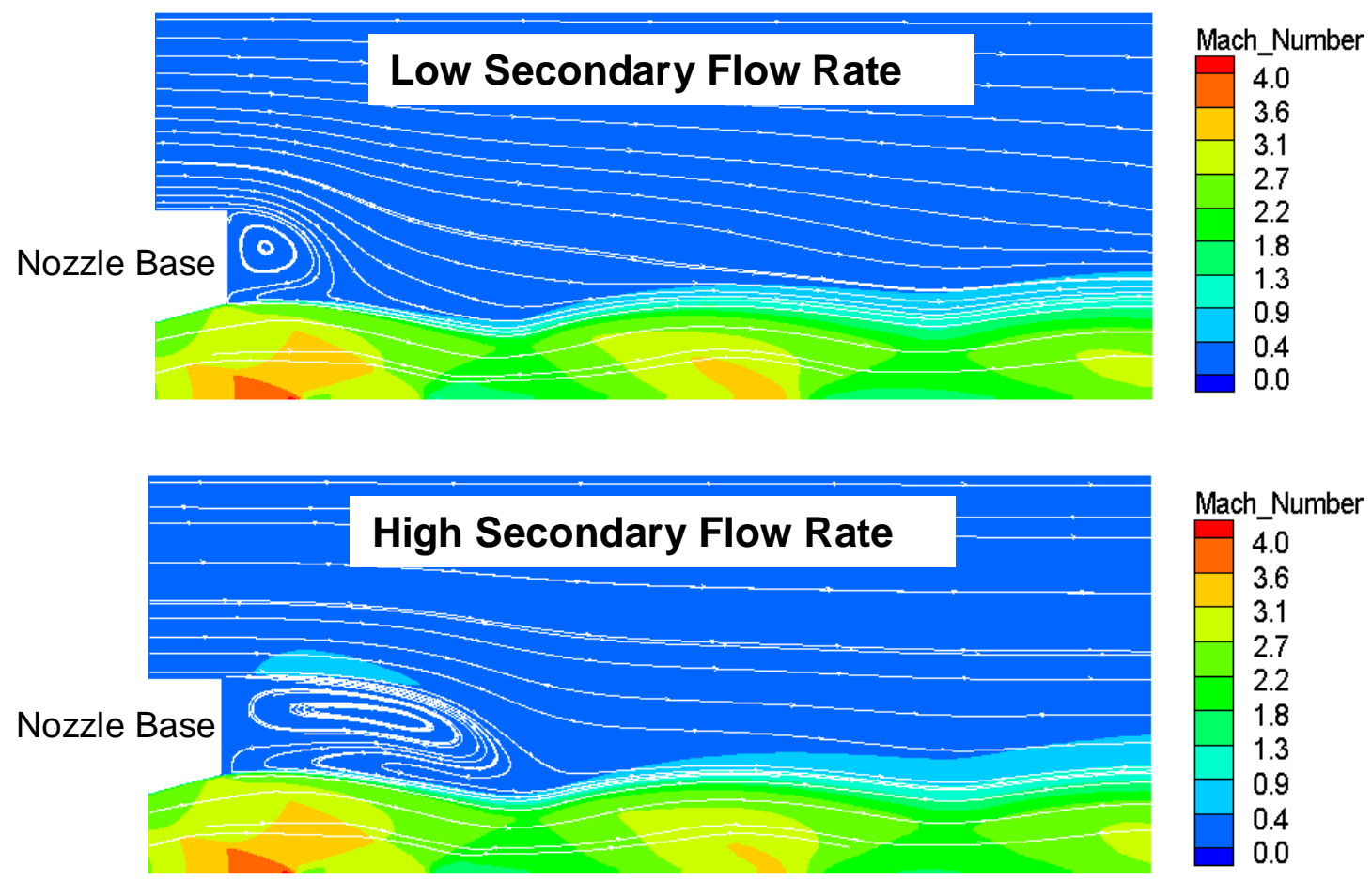

Figure 5-4 Mach Number and Streamlines from CFD Model ${ }^{30}$

The visual data acquired from the Gist and Purdue studies affirm that the CPSE simulation is capturing the correction trends in flow interactions. However, in order to numerically assess the accuracy of the CPSE simulation quantitative data such as entrainment ratios must be compared. Gist conducted a theoretical and experimental investigation of the influence of pressure ratio on entrainment ratio. Figure 5-5 shows the theoretical entrainment ratio distribution estimated by Gist. The saturated calculation presented previously in Equation 2.1 is used as a baseline to estimate the entrainment ratio, the ratio of secondary to primary mass flow rates. Gist then applies the empirical correction factor for primary plume growth represented by the different $K_{\text {exp and }}$ values. The $K_{\exp \text { and }}$ value of 1.0 represents ideal expansion of the primary plume with a correction for the thickness of the primary nozzle lip. The primary to secondary stagnation pressure ratio near 72 is the optimal expansion condition. 


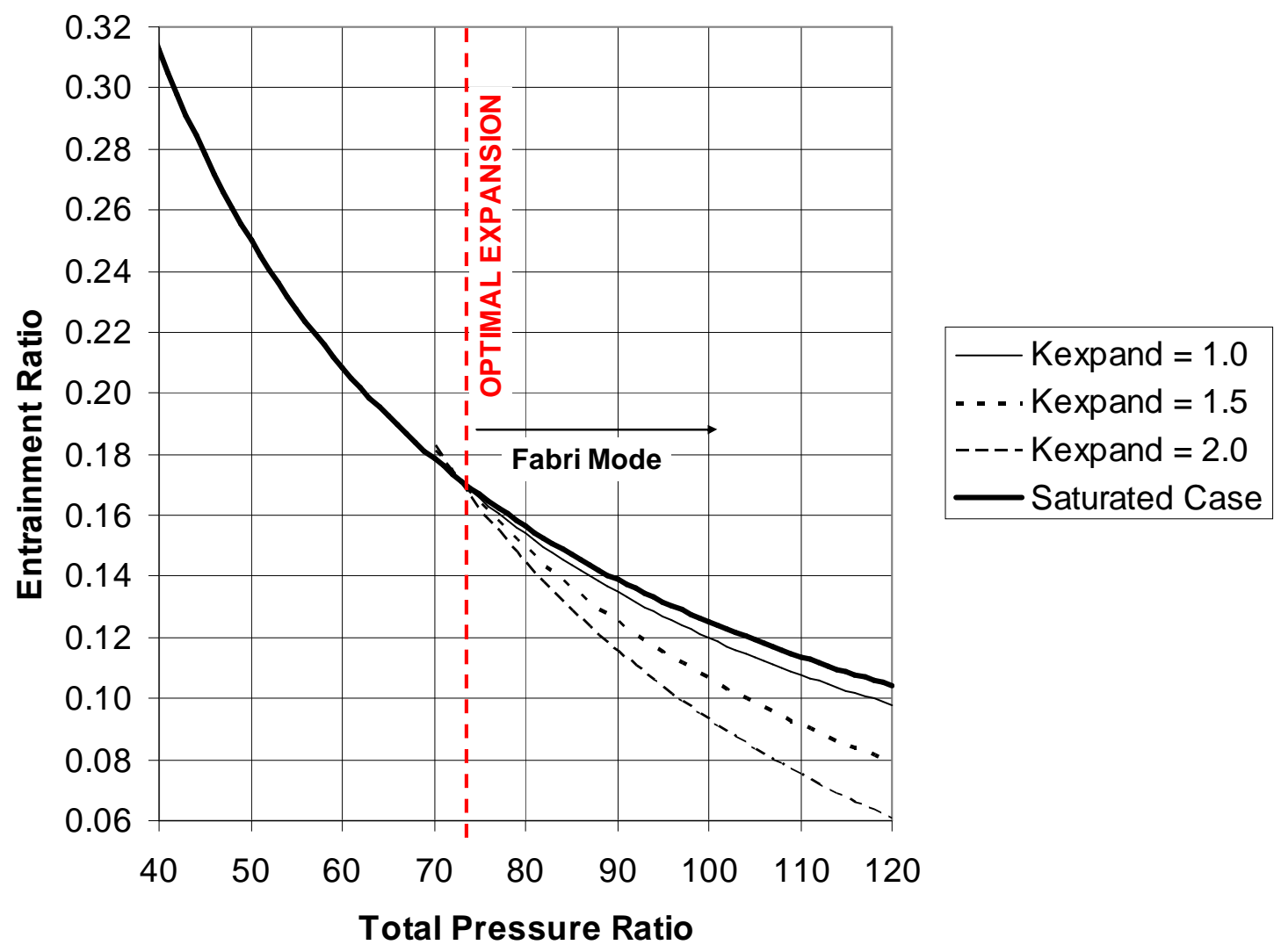

Figure 5-5 Entrainment ratio calculations using empirical correction factor for plume size $^{4}$

As the location of the optimal expansion suggests, higher entrainment ratios do not indicate better performance. Optimal performance occurs at the pressure ratio where the mass flow rate is maximum for the given ejector. Pressure ratios above the optimal condition result in a primary plume which expands into the secondary flow area. This reduced area of the secondary flow results in a decreased secondary mass flow rate. Pressure ratios below optimal conditions result in a saturated flow pattern with a higher entrainment ratio. This condition is suboptimal because the primary plume is not providing the maximum mass flow. Although the Mach number of the primary flow is dictated by the nozzle geometry, the mass flow of the primary plume is a function of the chamber pressure. The pressure ratio of optimal plume expansion yields the maximum mass flow of both the primary and secondary streams. 
Gist's empirical correction factors are only implemented at pressure ratios above that which yield optimal expansion. The empirical correction factors do not alter the entrainment ratio at pressure ratios below optimal expansion because the primary plume does not expand into the secondary flow area when operating in the saturated flow regime.

Figure 5-6 shows the lines of the theoretical approximations of Gist with the corresponding experimental results represented by blue asterisks. The experimental data points show some fluctuation, however, they correspond closely to a $K_{\exp \text { and }}$ value of 1.5. The approximation of Gist is able to closely predict the experimental data when the correction factor is implemented. However, a large amount of empirical knowledge is required to perform such an analysis. The proper $K_{\exp \text { and }}$ value for a specific geometry must be determined experimentally and the point of optimal expansion must also be known. An incorrect estimation of $K_{\exp \text { and }}$ can result in errors of over $20 \%$. 


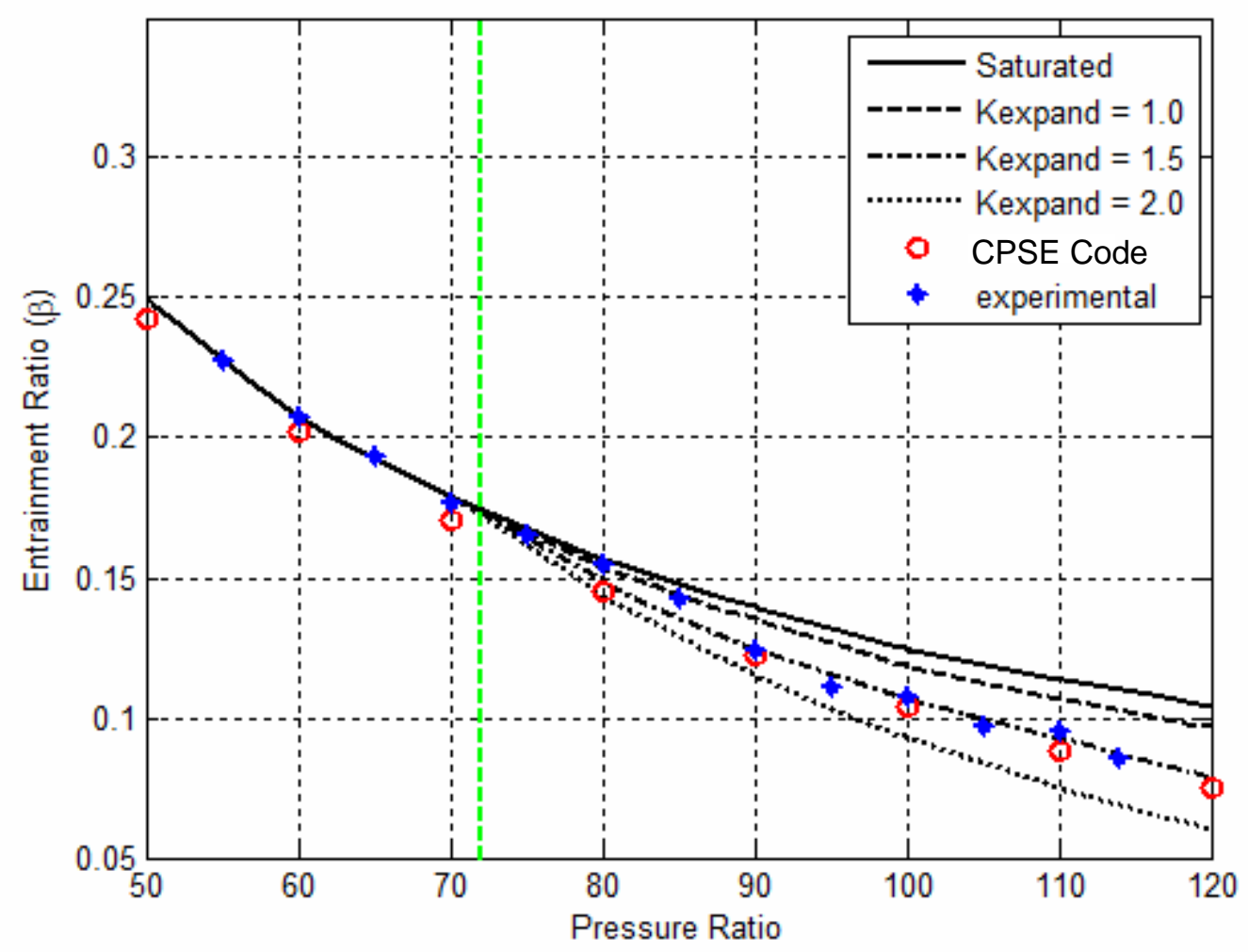

Figure 5-6 Comparison of CPSE computer code to experimental data and empirical predictions

Figure 5-6 also shows that Gist's experimental and theoretical results provide excellent validation for the numerical CPSE computer simulation. The red circles in the plot are data points from the CPSE computer simulation which very closely follow the trend of the data throughout the range of pressure ratios, despite fluctuations in the experimental data. The CPSE simulation does not implement any empirical correction factors or require any apriori knowledge to achieve this level of accuracy. The only parameters required by the CPSE simulation are the geometry of the ejector and the stagnation conditions of the primary and secondary streams. The average error between the CPSE numerical simulation and the experimental data is $3.67 \%$. In every case the CPSE code slightly under predicted the entrainment ratio. Some of this under prediction is likely due to the $3^{\circ}$ divergence in the duct walls of the CPSE experimental apparatus which is not in the CPSE simulation. However, it is estimated that by running more pressure ratios experimentally and in the simulation, the average error would be reduced without modification of the simulation. The noise in the experimental data suggests some 
unsteady operation of the ejector occurred. Gist suggests leakage and asymmetrical entrainment take place in some of the trials. More trials at each pressure ratio will provide experimental results with less noise that can be better used to evaluate the accuracy of the CPSE simulation. The future versions of the CPSE test apparatus will not have diverging duct walls which will aid in isolating likely the cause of the error. Some deviation may also occur due to the inviscid method of analysis used by the CPSE simulation. It is of interest to quantify the influence of viscous effects on the entrainment ratio.

\section{Conclusions}

The CPSE computer code is an automated simulation of the two-dimensional planar Cal Poly Supersonic Ejector. The application of the most relevance for this simulation is a rocket based combined cycle hypersonic propulsion system operating in the air augmented rocket mode for takeoff and acceleration in the subsonic flight regime. Hot fire combustion tests were conducted with the CPSE at low pressure ratios. Cold flow tests were run with higher pressure ratios. Both sets of tests entrained air from ambient conditions. The experiments with the higher pressure ratios were approximated by equations based on Fabri's quasi-one-dimensional method with an empirical correction factor added to increase accuracy. The CPSE simulation is a higher fidelity numerical approximation method without the need for empirical correction factors or apriori knowledge of the flow phenomena in the various aerodynamic flow patterns. The CPSE simulation was developed using the work of Addy as a foundation. The Method of Characteristics is used to describe the primary plume of the supersonic ejector with an initial guess for the pressure distribution of the secondary flow. The secondary flow properties and updated pressure distribution are solved using isentropic relations and the geometry of the primary plume determined by the Method of Characteristics. The

pressure distribution of the secondary flow is fed back into the Method of Characteristics and this process iterates until the solution of the primary and secondary streams converges. 
The Method of Characteristics provides the properties and geometry of the primary plume. These properties vary in the streamwise and transverse direction. The secondary stream is solved using isentropic relations and only varies in the streamwise direction. There are no viscous or thermo-chemical reactions taken into consideration in the simulation.

Saturated, supersonic Fabri choke and blocked aerodynamic flow patterns were seen in the CPSE simulation as the primary to secondary stagnation pressure ratio is increased. As the pressure ratio is increased, the entrainment ratio decreases. Optimal expansion occurs at the transition from the saturated case to the Fabri choke condition. During optimal expansion the ejector is operating most efficiently. The identification of the optimal expansion pressure ratio of 74 agrees with experimental data from Gist and Foster.

The presence of a weak shock in the primary plume emanating from the nozzle lip and a recirculation zone at the nozzle lip were detected in all aerodynamic flow patterns. The presence of the shock structure and recirculation zone at the nozzle lip is confirmed by previous experiments. The simulation suggests that as pressure ratio is increased, the compression shock decreases in strength and the recirculation zone shrinks in size. Both these phenomena are due to the primary plume boundary being able to expand more freely when the secondary stagnation pressure is lower. This trend of expansion causes the location of maximum primary plume expansion to move downstream as the pressure ratio is increased until the blocked case occurs. Once the blocked case occurs near a pressure ratio of 230 , the location of maximum primary plume expansion is dictated by the primary plume's intersection with the duct wall. Increasing the pressure ratio under this condition causes the streamwise location of the primary plume's intersection with the duct wall to move upstream. This unhindered expansion of the primary plume would not only decrease performance of air augmented ejector engines, but may also cause damage.

Using the Method of Characteristics for the primary supersonic plume and isentropic area relations for the secondary plume, the CPSE computer simulation is able to accurately 
predict entrainment ratios of supersonic air to air planar ejectors within $4 \%$. The accuracy of the simulation which has no viscous considerations suggests that ejectors operating with a high supersonic primary plume have flow phenomena which are governed by pressure distribution, not viscous effects. This rapid simulation algorithm can be implemented for preliminary design analysis and determining which cases should be examined using CFD and high fidelity methods. This simulation has already provided insight for modification of the CPSE experimental test rig. The CPSE simulation results have provided the required data for inlet sizing, plenum specifications and pressure transducer location of the next CPSE experimental test rig.

\section{Future Work}

Beyond the results presented within this paper, the CPSE computer simulation currently has parametric capability yet to be validated. Geometry changes such as nozzle curvature and dimensions, duct width, and duct wall contours are accepted by the simulation. Gas composition can be modified by changing the ratio of specific heats of the fluids. The stagnation temperatures of the gases may also be changed, although there are currently no heat transfer considerations implemented in the simulation.

The CPSE computer simulation is coded in a manner which readily accepts additional capability and fidelity. Several additions to the code are suggested. Viscous interactions between the secondary flow and the duct walls should be implemented to determine the effect of viscosity at different primary Mach numbers and primary to secondary stagnation pressure ratios. Viscous effects between the primary plume and the secondary flow should also be added to the capability of the CPSE simulation. These additions will make the secondary stream analysis into a full two-dimensional method which will provide much more realistic simulations of the flow phenomena occurring within the ejector. Thermal and chemical interactions would allow the CPSE code to better approximate the operation of an air augmented rocket operating with a fuel rich primary plume. Simulations of flows with a hot primary plume can be validated using the experimental results of Foster. However, these additions will increase computational time. A more efficient iteration scheme would reduce run time and benefit parametric or higher fidelity analyses. 
The parametric capability of the CPSE simulation already built into the code can be taken advantage of with more experimental data. The simulation cannot be used to its full capacity without a test apparatus which can vary geometry and expand the regime of pressure ratios. A variable geometry nozzle and movable duct walls would provide validation for various ejector geometries within the two-dimensional planar category. After an investigation is performed, the primary nozzle contour should be optimized for performance. The current primary nozzle contour is designed for ease of manufacturing. Furthermore, the addition of a low pressure plenum to the secondary flow inlet would dramatically increase the range of experimentally examinable pressure ratios.

\section{References}

1. Lear, W.E., Sherif, S.A., Parker, G.M., "Effects of Fabri Choking on the Performance of Two-Phase Jet Pumps", AIAA 3012, January 2000.

2. Fung Sang Kang, Andrew, "Modeling of Mixer-Ejector Nozzle Flows" Massachusetts Institute of Technology. February 1995.

3. Cutler, A.D., Magnotti, G., "Supersonic Combusting Jet Experiments for Code Development and Validation" Defense Test Resource Management Center, Hypersonic Test Focus Area, NASA Fundamental Aeronautics Hypersonics Program.

4. Gist, Ryan, "Examination of Flow Field Characteristics and Fabri-Choking of a 2D Air Augmented Rocket” California Polytechnic State University, January 2007.

5. Curry, Marty, "NASA Dryden Hyper-X/X43-A Graphics Collection", http://www.dfrc.nasa.gov/Gallery/Graphics/Hyper-X/Large/X-43A 3viewcolor.jpg [retrieved April 3 2010].

6. Federation of American Scientists, "X-43 Hyper X Program", http://www.fas.org/programs/ssp/man/uswpns/air/xplanes/x43.html [retrieved April 3 2010].

7. Curry, Marty, "NASA Dryden SR-71 Graphics Collection", http://www.dfrc.nasa.gov/Gallery/graphics/SR-71/index.html [retrieved April 3 2010].

8. Warwick, Graham, "DARPA Cancels Hypersonic Blackswift", Aviation Week [online], October 13, 2008.

http://www.aviationweek.com/aw/generic/story.jsp?id=news/BLACK101308.xml $\underline{\text { \&headline}=\mathrm{DARPA} \% 20 \mathrm{Cancels} \% 20 \mathrm{Hypersonic} \% 20 \mathrm{Blackswift} \& \text { channel=defens }}$ e [retrieved April 7 2010].

9. Albertson, Cindy W., Emami, Saied, Trexler, Carl A., "Mach 4 Test Results of a Dual-Flowpath, Turbine Based Combined Cycle Inlet", NASA Langley Research Center, Hampton, Va.

10. Foster, Trevor James, "Rectangular Ducted Methane/GOX Thruster", February 2008. California Polytechnic State University. 
11. Papamoschou, D., "Analysis of Partially Mixed Supersonic Ejectors", Journal of Propulsion and Power, Vol. 12, No. 4, July 1996.

12. Fabri, J., and Siestrunk, Supersonic Air Ejectors", Advances in Applied Mechanics, Vol. 5, Von Karman and Dryden, editors, Academic Press, 1958, pg $1-35$.

13. Fabri, J., \& Paulon, J., "Theory and Experiments on Supersonic Air-to-Air Ejectors", NACA TM 1410, Sept 1956.

14. Addy, L.A., "On the Steady State and Transient Operating Characteristics of Long Cylindrical Shroud Supersonic Ejectors", University of Illinios, Ph. D. dissertation. University Microfilms, Inc. Ann Arbor, MI., 1963.

15. Hall, J.L., Dimotakis,P.E., and Rosemann, H., "Experiments in Non-Reacting Compressible Shear Layers, AIAA-1991-0629, 29th Aerospace Sciences Meeting, Reno, 1991.

16. Grosche, C.E., Seiner,J.M.,Hussaini, M.Y., Jackson,T.L., "Numerical Simulation of Mixing Enhancement in a Hot Supersonic Jet."

17. Seider, Warren D., Churchill, Stuart W., "Confined Jet Mixing in the Entrance of a Tubular Reactor", AlChE Journal, University of Michigan, May 1971.

18. Emanuel, N.G., "Comparison of One-Dimensional Solutions with Fabri Theory for Ejectors", Acta Mechanica 44, pgs. 187-200, 1982.

19. Huang, B.J., Chang, J.M., Wang, C.P., Petrenko, V.A., "A 1-D analysis of ejector performance", International Journal of Refrigeration 22 (1999) 354-364.

Department of Mechanical Engineering, National Taiwan University.

20. Mickelson, C.D, Sandberg , M.R, Addy, A.L, "Theoretical and Experimental Analysis of the Constant Area Supersonic-Supersonic MixerEjector", October 1976.

21. Cutler, A.D., Carty, A.A., Doerner, S.E., Diskin, G.S., and Drummond J.P., "Supersonic Coaxial Jet Experiment for CFD Code Validation", AIAA-99-3588, 30th AIAA Fluid Dynamics Conference, Norfolk, 1999.

22. Simonson, A.J., Schmeer, J.W., "Static Thrust Augmentation of a Rocket-Ejector System with a Heated Supersonic Primary Jet", NASA TN D-1261, May 1962.

23. Zucrow, Maurice J., Hoffman, Joe D., Gas Dynamics Volume I. 1976. John Wiley and Sons, Inc. New York

24. Zucrow, Maurice J., Hoffman, Joe D., Gas Dynamics Volume II: Multidimensional Flows. 1977. John Wiley and Sons, Inc. New York

25. Riley, Paul. "Axisymmetric Method of Characteristics: An Algorithm and Its Validation", California Polytechnic State University, June 13, 2008

26. De Chant, L.J. \& Canton, J.A., "Measurement of Confined Supersonic, 2-D JetLengths Using the Hydraulic Analogy", Experiments in Fluids, Vol. 24, pgs. 5865, Springer-Verlag, 1998.

27. Kouchi, T., Kobayashi, K., Murakami, A., Kato, K., Tomioka, S., "Performance of a RBCC Combustor Operating in Ramjet Mode", AIAA 2006-4867, 42nd AIAA Joint Propulsion Conference, Sacramento, 2006.

28. Kobayashi, K., Tomioka, S., Kato, K., Murakami, A., and Kudo, K., "Performance of a Dual-Mode Combustor with Multistaged Fuel Injection", Journal of Propulsion and Power, Vol. 22, No. 3, 2006. 
29. Dimotakis, P.E., "Turbulent Free Shear Layer Mixing and Combustion" in Murthy, S.N.B., Curran, E.T., editors, High-Speed Flight Propulsion Systems, Progress in Astronautics and Aeronautics, Volume 137, pgs 265-340.

30. Batt, R.G., "Turbulent Mixing of Passive and Chemically Reacting Species in a Low-Speed Shear Layer", Journal of Fluid Mechanics, Vol. 82, pp 53-95, Aug 1977.

31. Gujarathi, A., Li, D., Anderson, W., \& Sankaran, V. "CFD Modeling of a Ducted Rocket Combined with a Fuel-Rich Primary Thruster", $42^{\text {nd }}$ Joint Propulsion Conference. AIAA 4577, July 2006 


\section{Appendix}

-Cal Poly Air Augmented Rocket Code

\%formulated and Coded by Brett Morham

\%January 14,2009

$\circ$

oMethod of Characteristics provided by Paul Riley

$\mathrm{ClC}$

close all

clear

format compact

tic

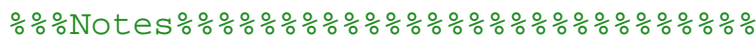

$\% \frac{\circ}{0} \%$ Fabriblock at $\mathrm{PR}>230 \% \% \frac{0}{0} \% \frac{0}{0} \% \frac{0}{0} \% \frac{0}{0} \%$

응은

velocities w/in iteration scheme)

$\frac{0}{0} \% \frac{0}{0}$ Input Geometric properties of the ejector $\% \% \frac{0}{0}$

응응№zzle Geometry \& Thermodynamic Properties $0 \% \frac{0}{0} \circ \% \circ \%$

theta_max $=15.35 ; \% 15.35 ;$ \%max turning angle (degrees)

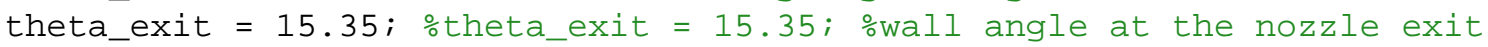
(degrees)

r_th $=.05 / 12 ;$ \% r_th $=.05 / 12 ;$ othroat radius (ft)

zlen $=1.65 / 12 ;$ \%len $=0.3417$; \%nozzle length (ft)

$\mathrm{POP}=1750 * 144 ; \% 1690 * 144 ; \% \mathrm{POP}=1350 * 144 ; \%$ \%

psiochamber stagnation pressure (psf)

$\mathrm{TOp}=521.7 ; \circ 2000+475 ; \quad \%$ chamber stagnation temperature (deg R)

roc_u $=.22 ; \% .1 ;$ (ft) radius_of_curvature_upstream

roc_d $=.1 ; \% .10 ; \%$ roc_d $=0.090695 ; \%$ (ft)

radius_of_curvature_downstream

gamma $=1.4 ; \circ 1.24$; \%ratio of specific heats for the working fluid

$\mathrm{R}=1774.864 ; \%$ ㅇas constant for the primary flow (ft-lbf/slug R)

delta $=0 ; \% 0$ for planar, 1 for axisymmetric

zduct $=14 / 12 ; \% 7.9$ inches from nozzle lip oduct length [feet] from

exit of nozzle.

rduct $=2.75 * 0.5 / 12$; ohalf duct width [ft] \%this need to become a

function

tb $=0.25 / 12 ;$ othickness of nozzle lip [ft]

thetaduct $=0 ;$ oangle of wall divergence in degrees. if wall converges use negative angle

$\mathrm{Ta}=528.7$; \%stagnation temperature of secondary flow [Rankine]

rhoa $=.002102$; stagnation density of seconday flow [slug/ft^3]

gammaa $=1.4 ;$ oratio of specific heats of secondary flow.

Rs = 1716; \%Gas constant for secondary flow [ft $2 / \mathrm{s} 2 * \mathrm{R}$ ]

A_inlet $=6 / 12$; $\frac{\circ}{2}$ rea of secondary inlet (must divide by duct height) [in]

$\mathrm{Pa}=6 * 144 ;$ ostagnation pressure of secondary flow [psf] 


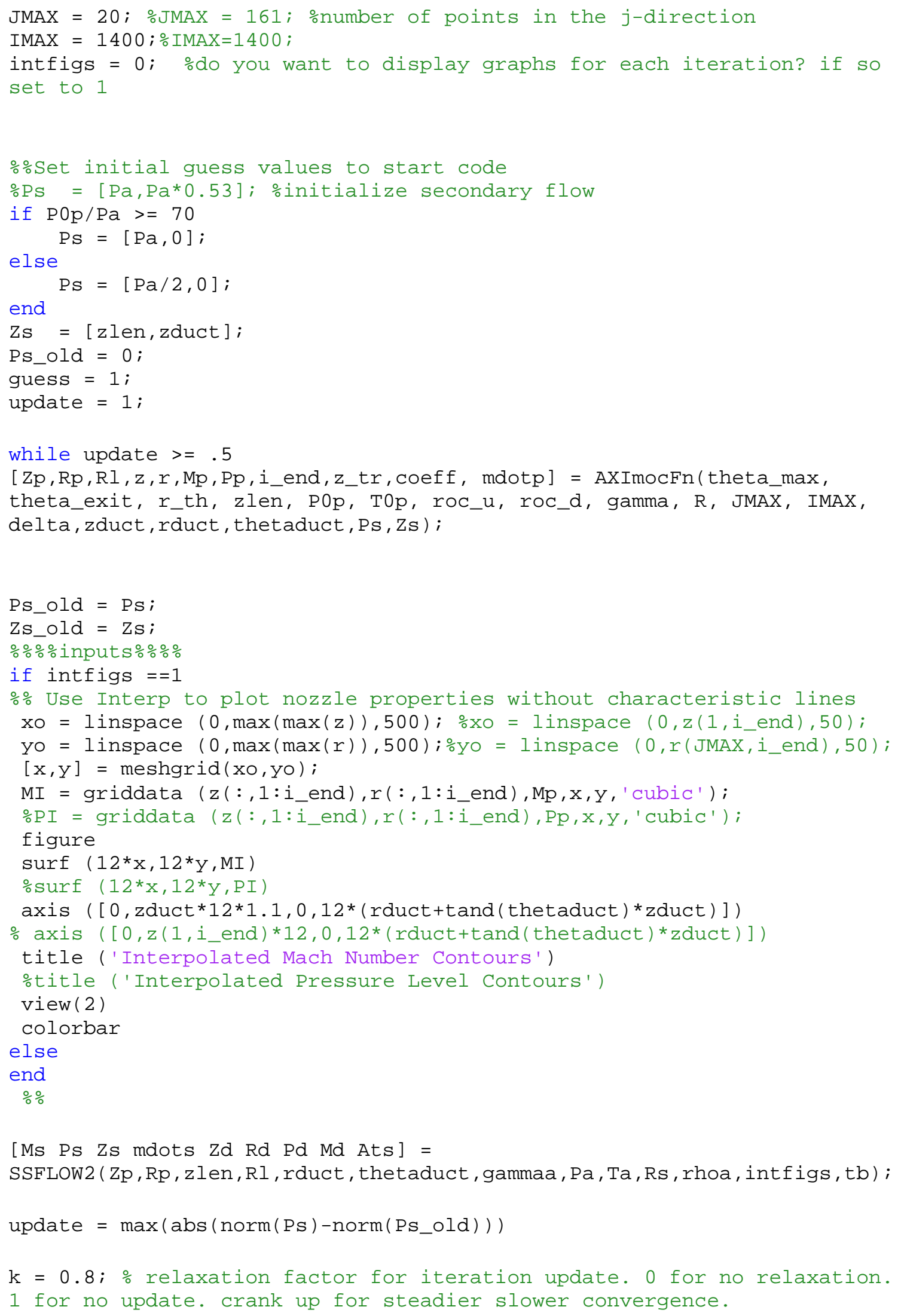




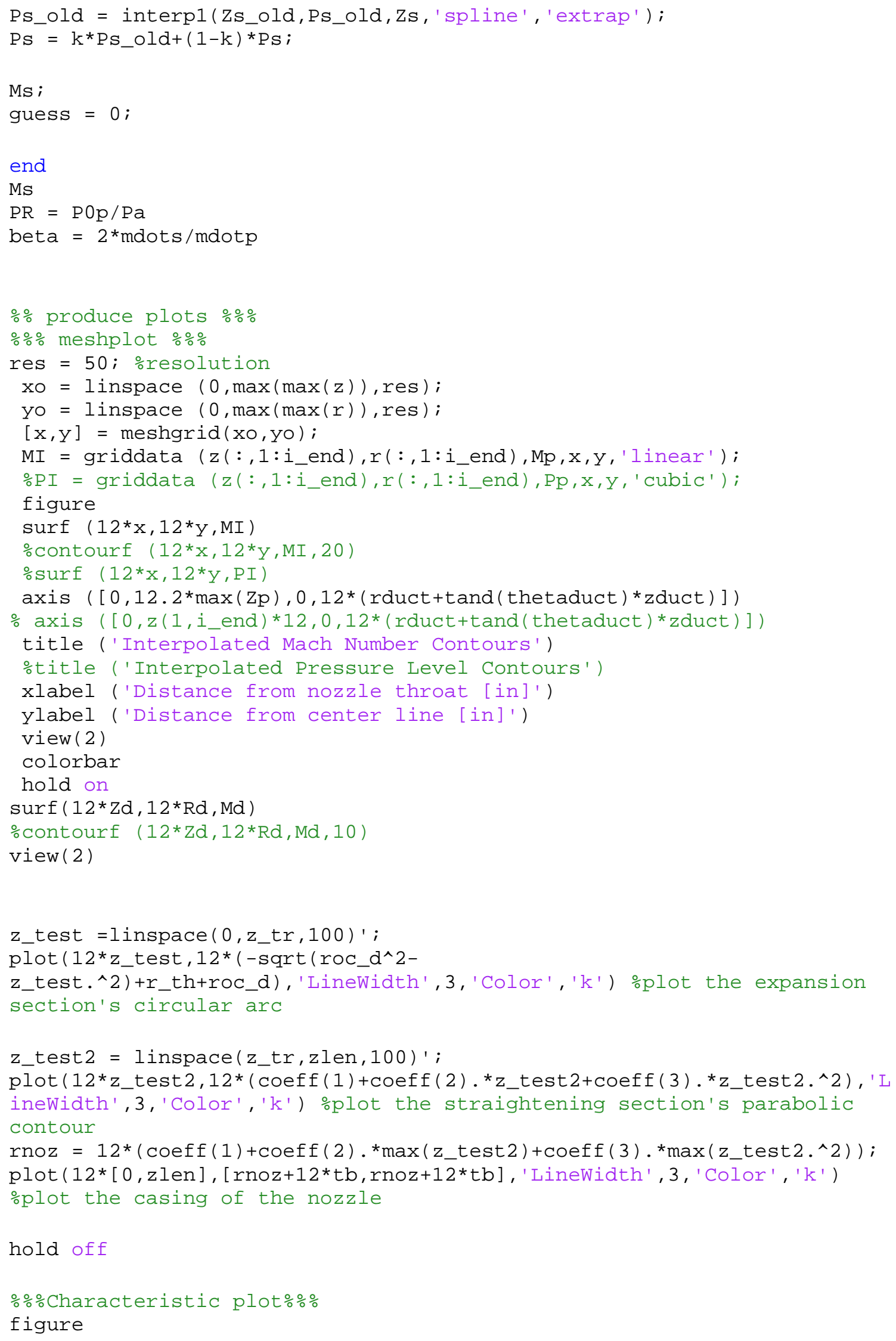


hold on

$\operatorname{surf}(12 * z(:, 1:$ i_end $), 12 * r(:, 1:$ i_end $), \mathrm{Mp})$

title('Mach contours')

view (2)

colorbar

z_test $=$ linspace $\left(0, z_{-}\right.$tr, 100) ';

plot $(12 * z$ _test, $12 *(-\operatorname{sqrt}($ roc_d^2-

z_test.^2)+r_th+roc_d), 'LineWidth', 3, 'Color', 'g') oplot the expansion

section's circular arc

z_test 2 = linspace $\left(z \_t r, z l e n, 100\right)$ ';

plot $\left(12 * z\right.$ _test $2,12 *\left(\operatorname{coeff}(1)+\operatorname{coeff}(2) \cdot{ }^{\star} z\right.$ test $\left.2+\operatorname{coeff}(3) .{ }^{*} z \_t e s t 2 . \wedge 2\right), ' \mathrm{~L}$ ineWidth', 3, 'Color', 'g') oplot the straightening section's parabolic contour

hold off

$\div \frac{\circ}{0}$

toc

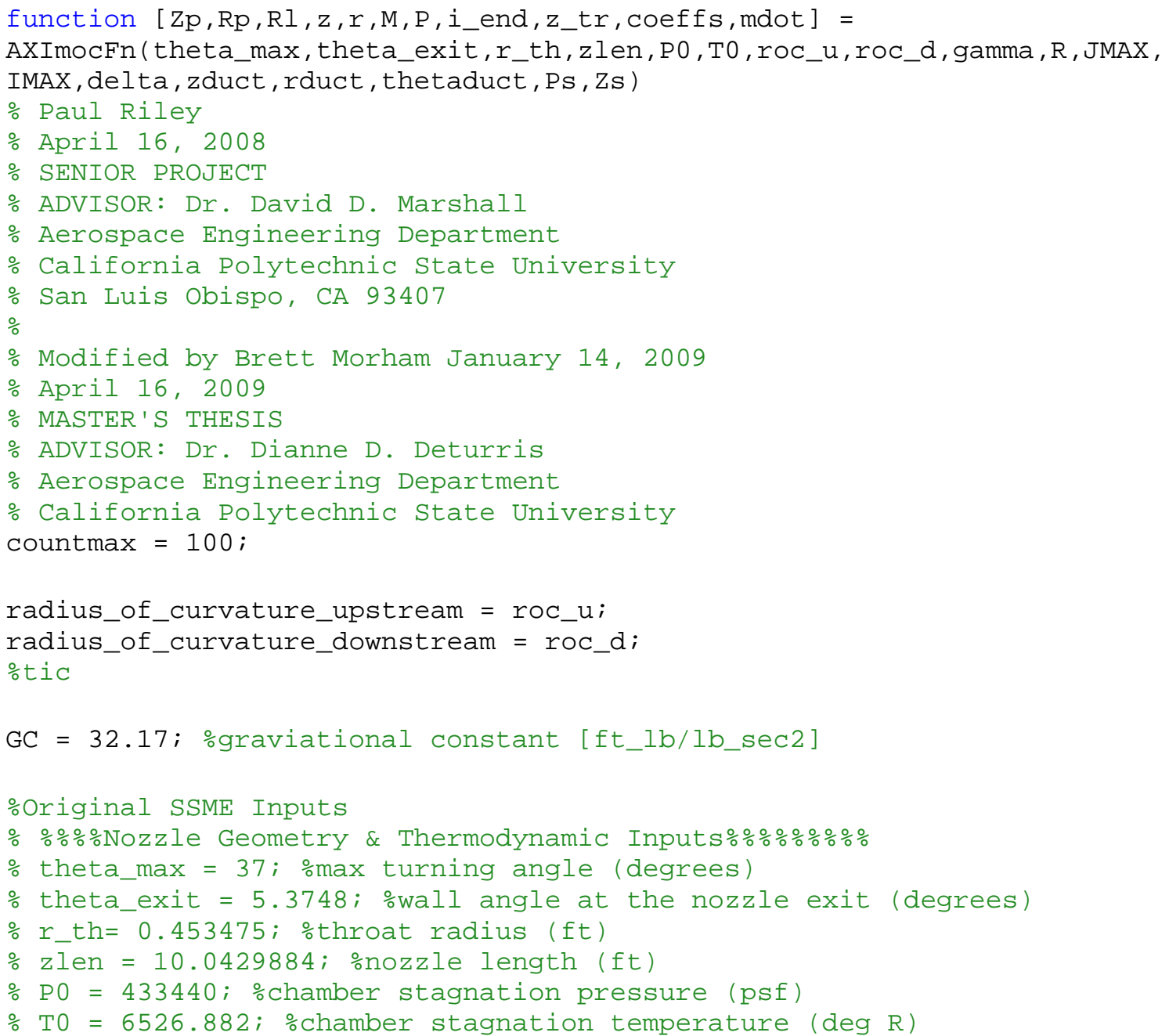


$\%$ radius_of_curvature_upstream $=0.22401665 ;$ \% (ft)

\% radius_of_curvature_downstream $=0.090695 ;$ \% (ft)

\% gamma=1.24; \%ratio of specific heats for the working fluid

$\circ R=3658.725$; $\%$ gas constant for the working fluid (ft-lbf/slug R)

\% delta $=1$ for axisymetric or 0 for planar(2D)

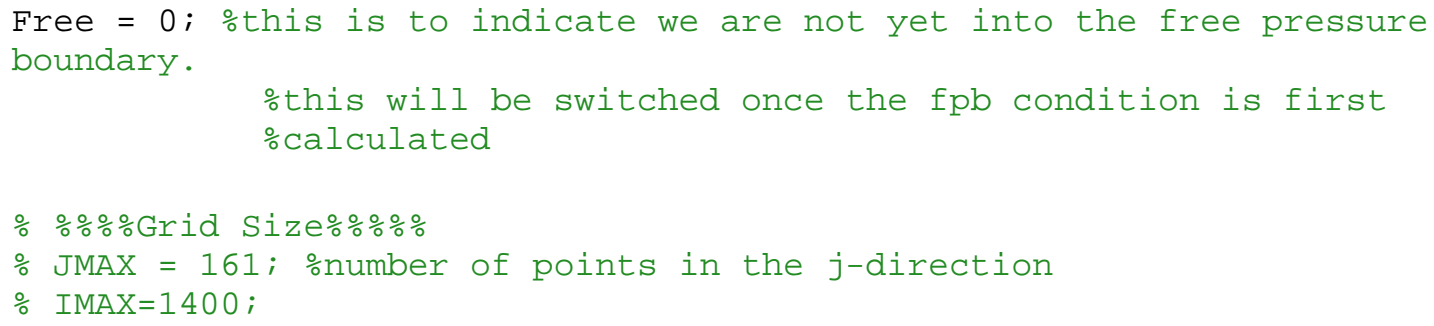

z_tr = radius_of_curvature_downstream*sind(theta_max); $\%$-coordinate of the wall point that connects the expanding and unexpanding wall contours $r \_t r=r \_t h+(1-\operatorname{cosd}($ theta_max $))$ *radius_of_curvature_downstream; \%rcoordinate of the wall point that connects the expanding and unexpanding wall contours

ocalculate the coefficients of the wall contour for the bell (or o"diminishing wall angle") region. Assuming a parabolic contour 으 $=a+b^{*} z+c^{*} z^{\wedge} 2$, the coefficients are: 
[x y u_tilda v_tilda] $=$

KliegelDataLine (gamma, R, radius_of_curvature_upstream, r_th, T0, JMAX) ;

$\mathrm{z}(:, 1)=\mathrm{x}$;

$r(:, 1)=y$;

$u(:, 1)=$ u_tilda;

$\mathrm{v}(:, 1)=\mathrm{v} \_$tilda;

done=false; onot done

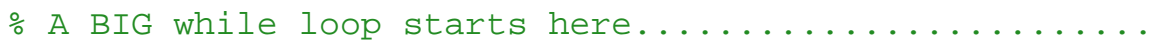

while ( done)

$i=i+1 ;$ ostart off with the second column

for $j=1: J M A X$

$u(j, i)=0$; $\quad$ oset arbitrary values for these variables so the code can continue

$\begin{array}{ll}u \_ \text {new }(j, i) & =75 ; \\ \mathrm{v}(j, i) & =0 ; \\ \mathrm{v} \_ \text {new }(j, i) & =75 ; \\ \mathrm{r}(j, i) & =0 ; \\ \text { r_new }(j, i) & =75 ; \\ \mathrm{z}(j, i) & =0 ; \\ \text { z_new }(j, i) & =75 ;\end{array}$

evencol $=(\bmod (i, 2)==0) ;$ oThis identifies an even column by saying if you divide the column number i by 2, the remainder must be zero.

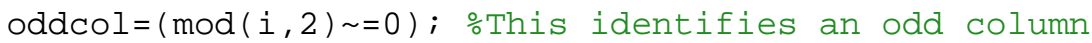

\% need common way of indexing to previous

\% column's characteristic points, but indexing

o depends on whether we are on an even or odd

\% column

\% jtop is the j-index of previous column point jtop: c-

starts

\% that $\mathrm{C}-$ characteristic starts

\% jbot is the j-index of previous column point jbot: C+

starts

\% that $\mathrm{C}+$ characteristic starts

if (evencol)

jtop $=j+1$

$j \mathrm{bot}=j$;

else

jtop $=j$;

joot $=j-1$

end

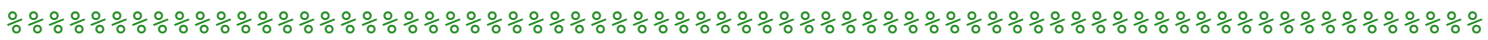

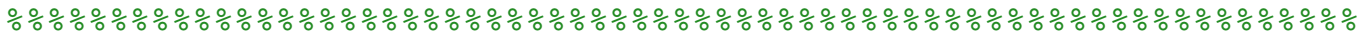




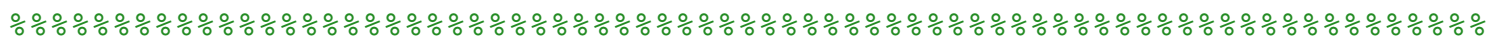

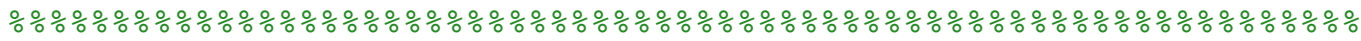
SUBSEQUENT COLUMNS- now that the initial column of points has been oestablished, complete the calculations for the remaining columns.

- What to do depends on which j-index and column (even or odd) you are working with

$\operatorname{switch}(j)$

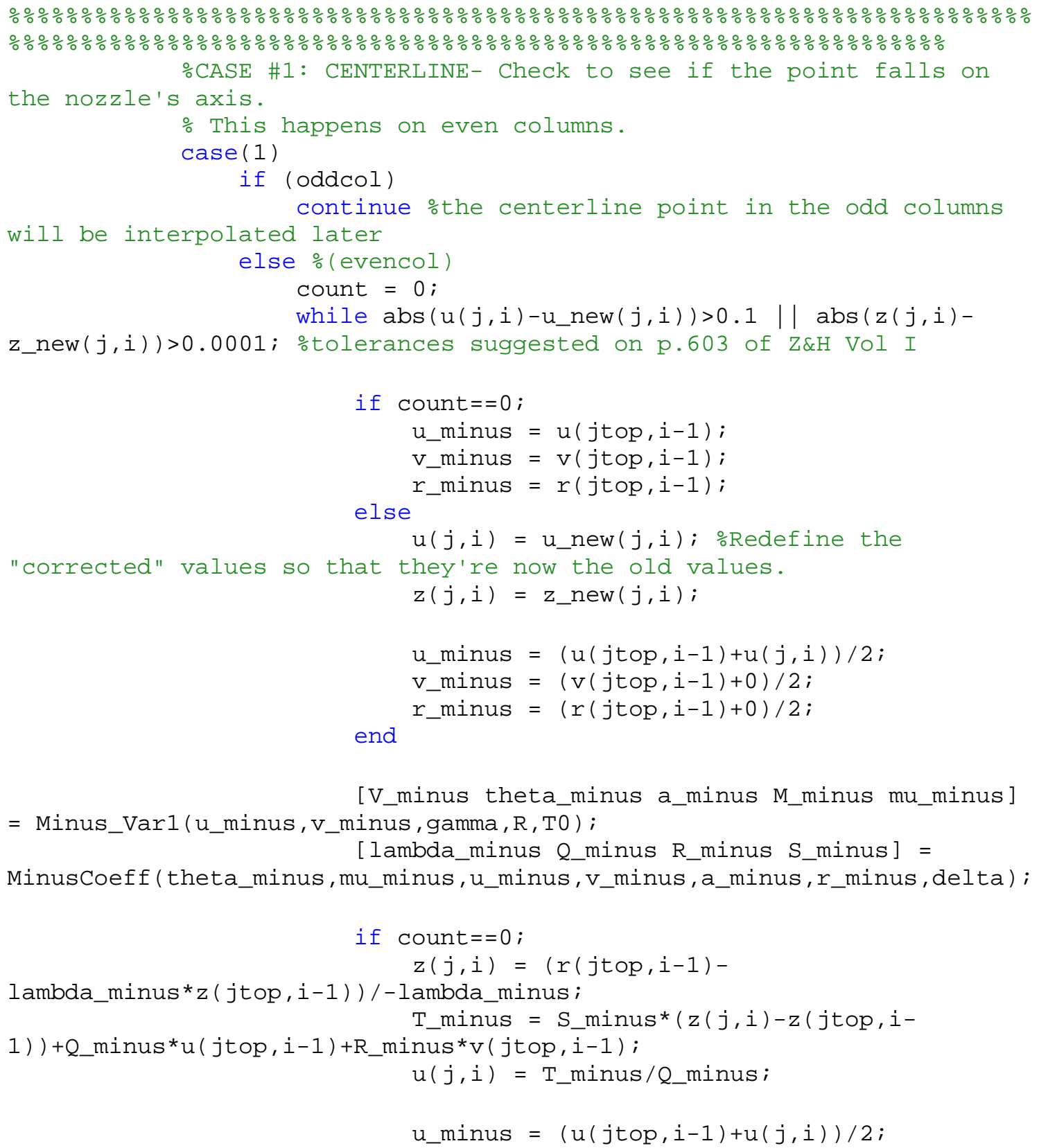




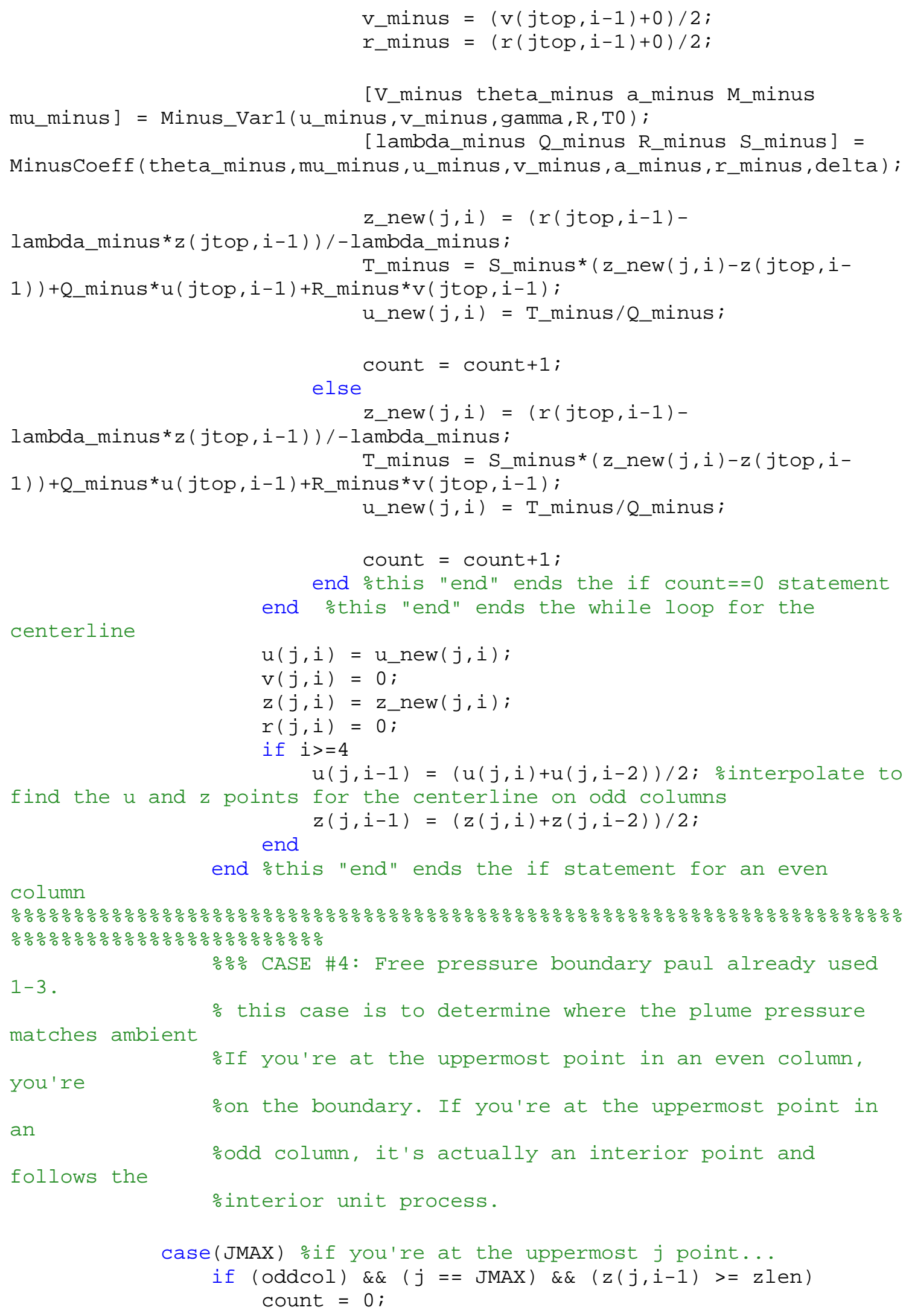


while abs $\left(u(j, i)-u \_n e w(j, i)\right)>0.1|| \operatorname{abs}(v(j, i)-$ $\left.\operatorname{v\_ new}(j, i)\right)>0.1|| \operatorname{abs}\left(r(j, i)-r \_n e w(j, i)\right)>0.0001|| \operatorname{abs}(z(j, i)-$ $z \_$new $\left.(j, i)\right)>0.0001$; \%tolerances suggested on p.603 of Z\&H Vol I

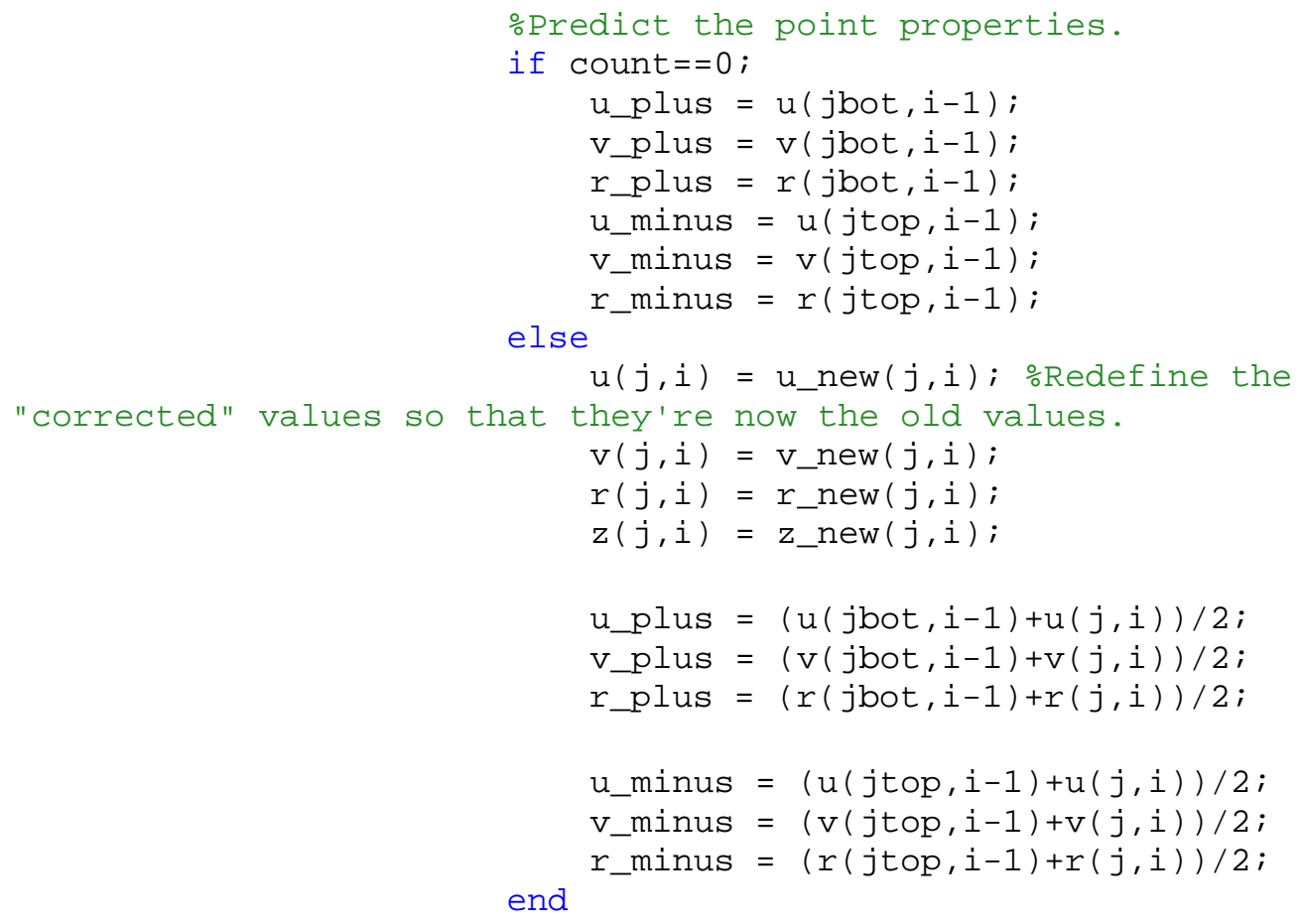




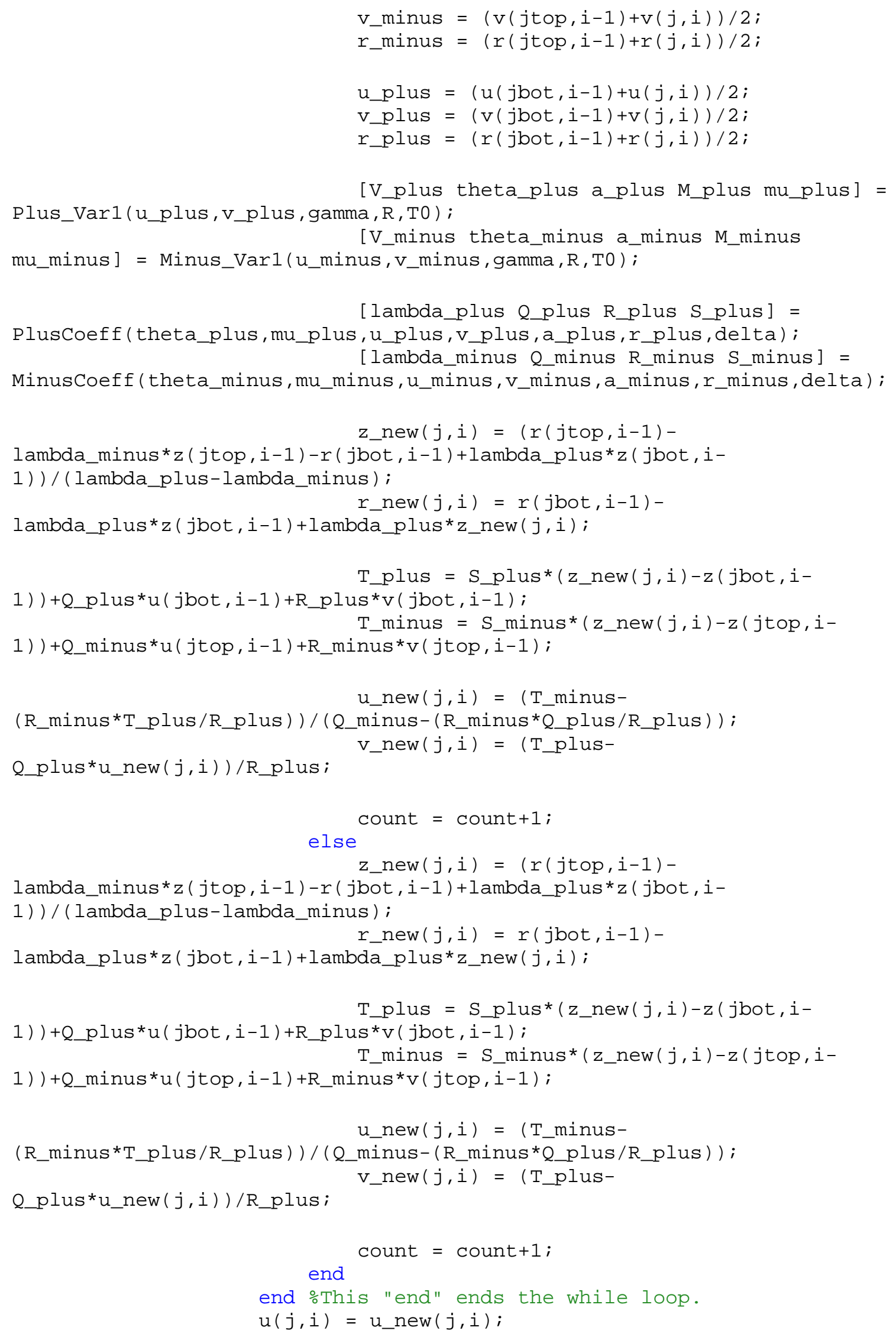




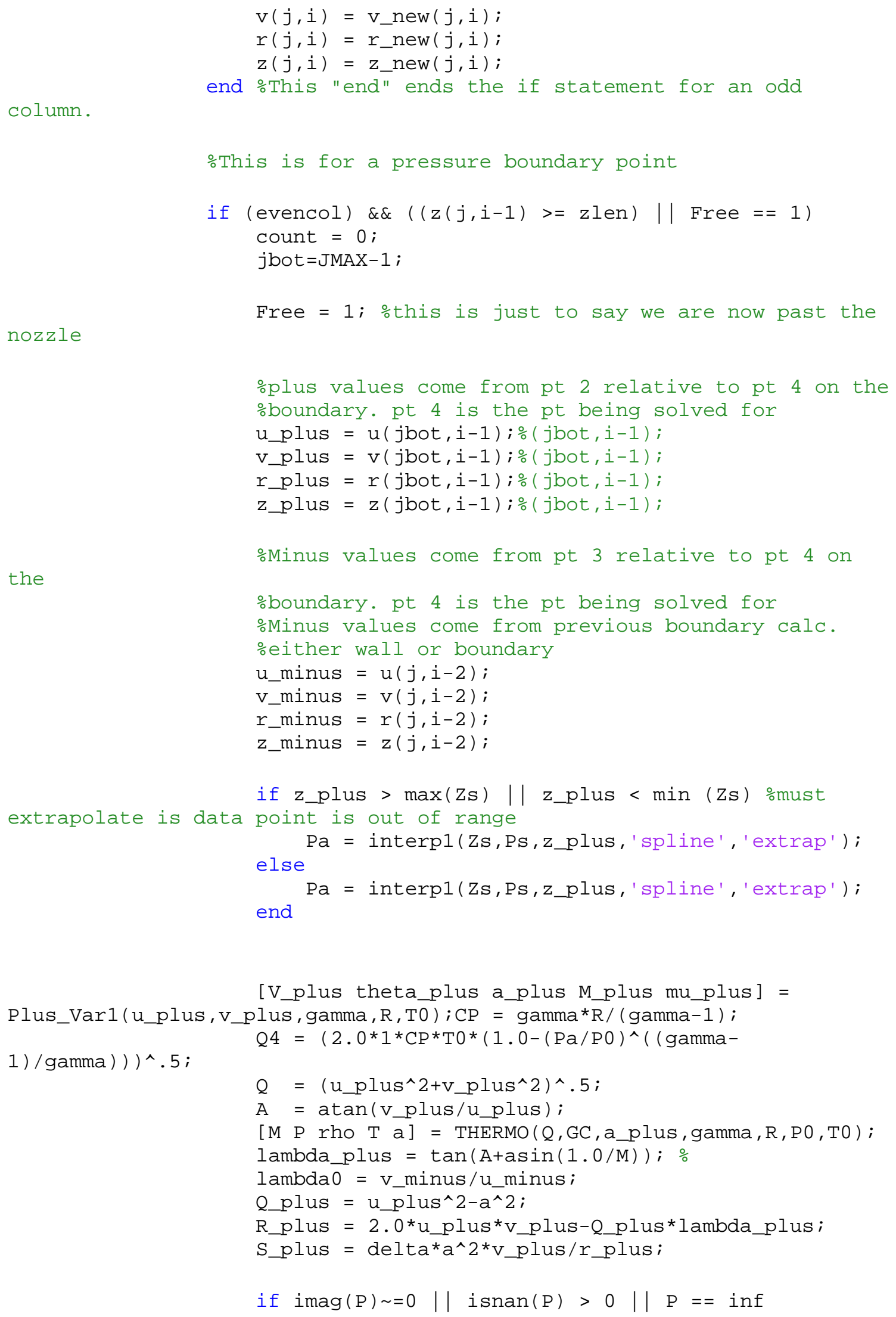




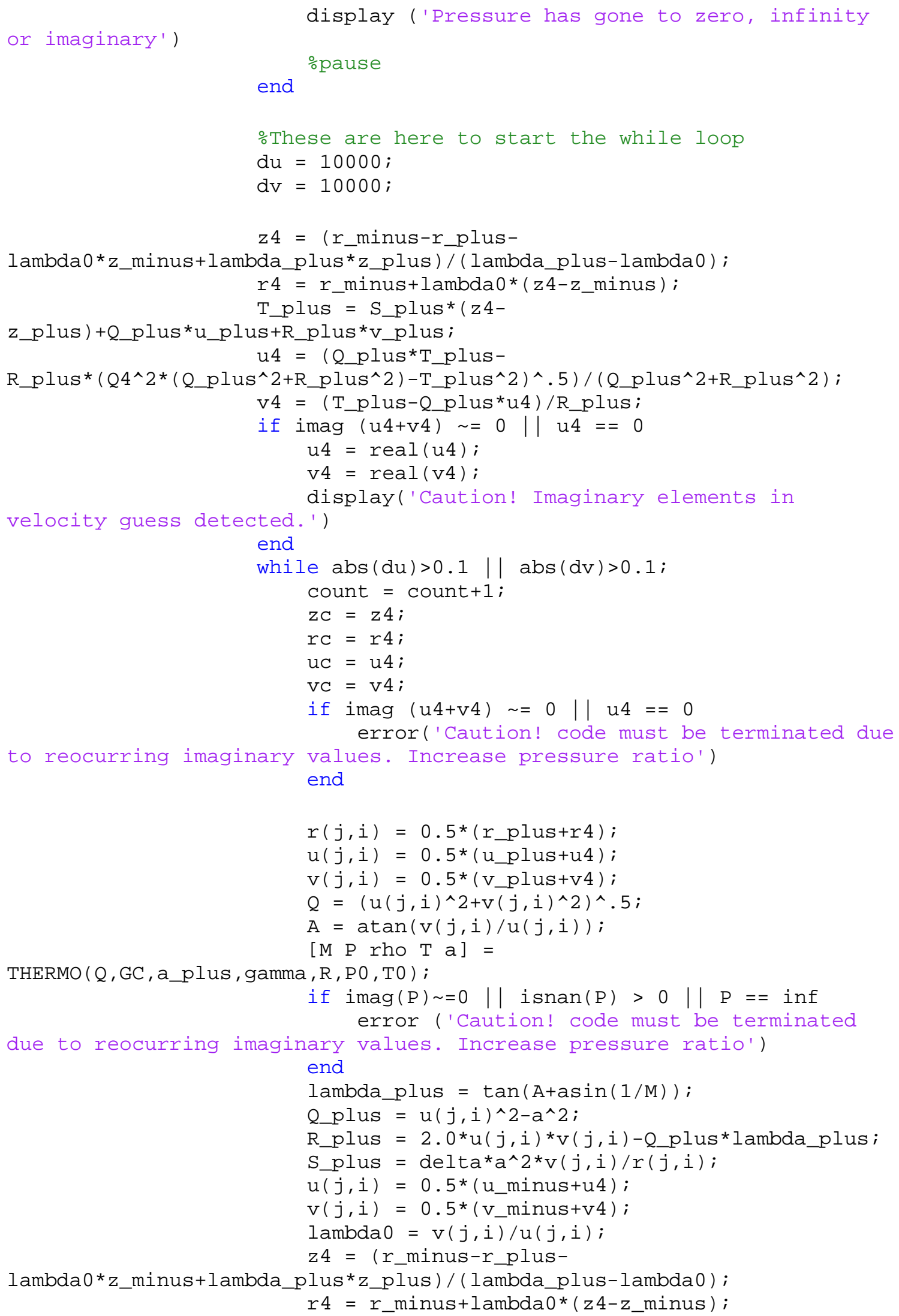




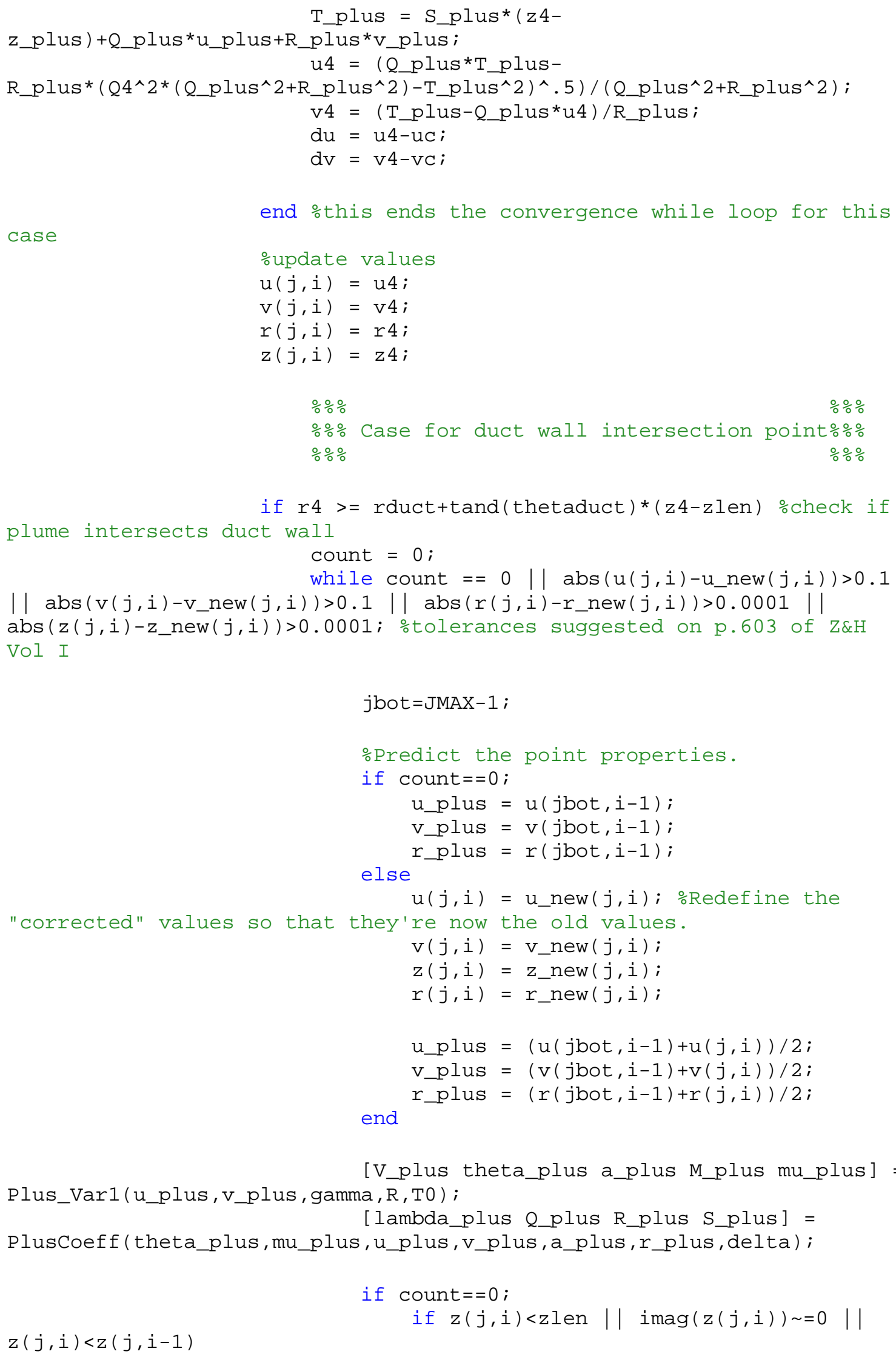




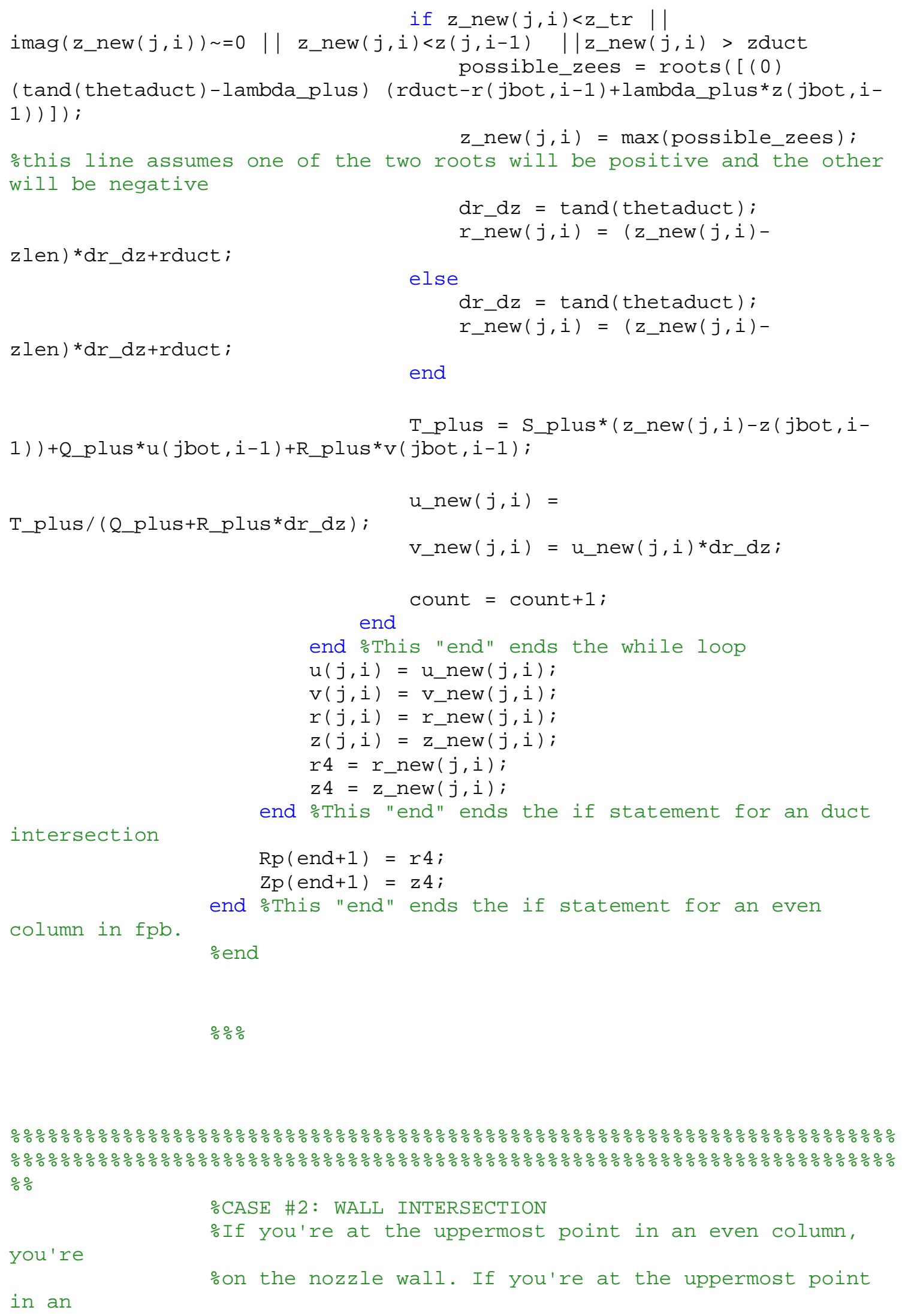


follows the

oodd column, it's actually an interior point and

ointerior unit process.

ocase (JMAX) \%if you're at the uppermost $j$ point...

if $($ oddcol $) \& \&(z(j, i-1)<z l e n)$

count $=0$;

while abs $\left(u(j, i)-u \_n e w(j, i)\right)>0.1|| \operatorname{abs}(v(j, i)-$

$\left.\operatorname{v\_ new}(j, i)\right)>0.1|| \operatorname{abs}\left(r(j, i)-r \_n e w(j, i)\right)>0.0001|| \operatorname{abs}(z(j, i)-$

$\left.z \_n e w(j, i)\right)>0.0001$; otolerances suggested on p.603 of $\mathrm{z} \& \mathrm{H}$ Vol I

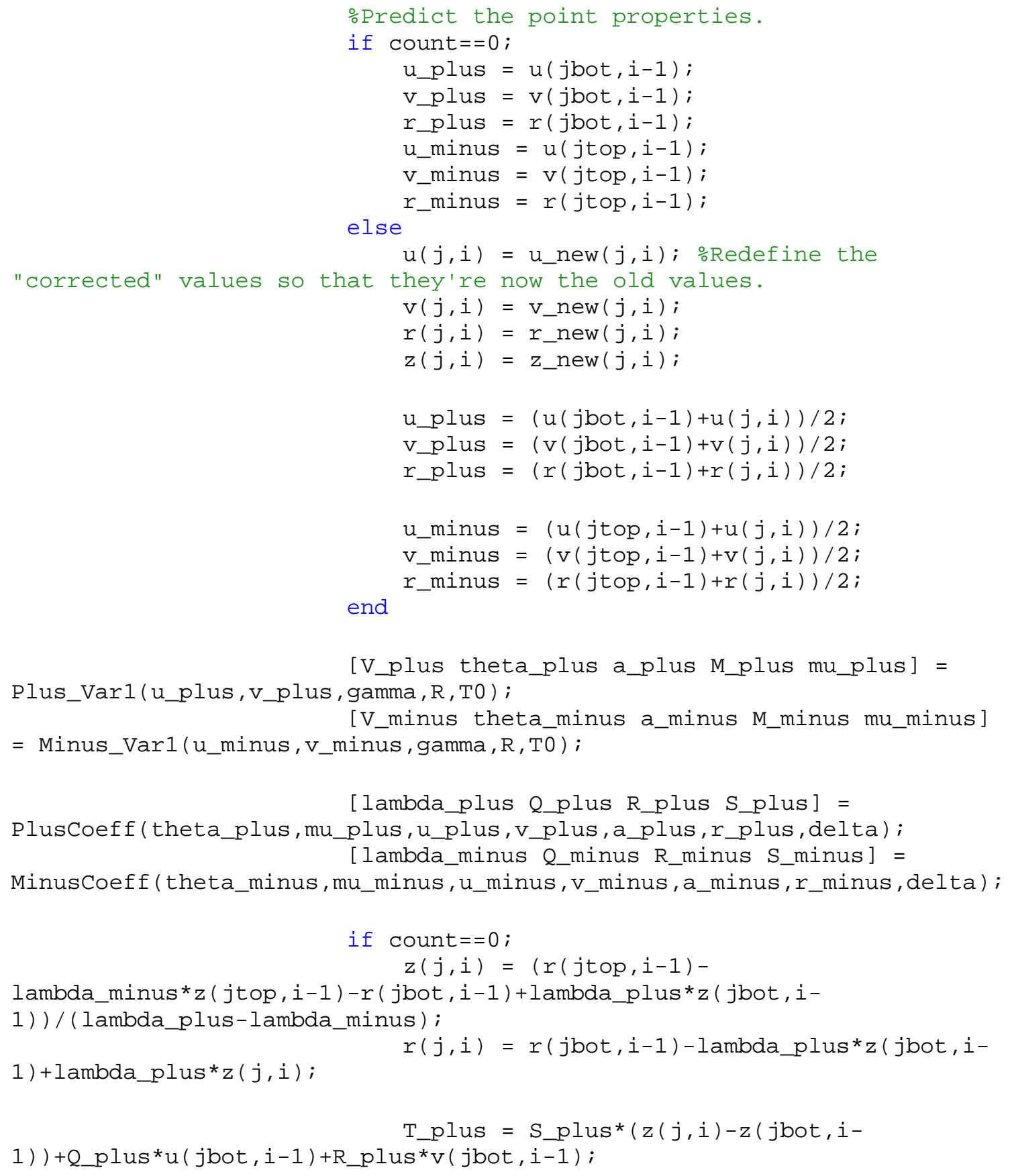


T_minus $=S \_$minus* $(z(j, i)-z(j t o p, i-$

1)) $+Q \_m i n u s * u(j t o p, i-1)+R \_m i n u s * v(j t o p, \bar{i}-1)$;

$u(j, i)=\left(T_{-}\right.$minus-

(R_minus*T_plus/R_plus)) / (Q_minus-(R_minus*Q_plus/R_plus)) ;

$v(j, i)=\left(T \_p l u s-Q \_p l u s * u(j, i)\right) / R \_p l u s ;$

u_minus $=(u(j$ top,$i-1)+u(j, i)) / 2$;

v_minus $=(v(j$ top,$i-1)+v(j, i)) / 2$;

$r \_$minus $=(r(j$ top,$i-1)+r(j, i)) / 2$;

u_plus $=(u(j$ bot,$i-1)+u(j, i)) / 2$;

v_plus $=(v(j$ bot,$i-1)+v(j, i)) / 2$;

$r \_p l u s=(r(j b o t, i-1)+r(j, i)) / 2$;

Plus_Var1 (u_plus, v_plus, gamma, R, T0) ;

[V_plus theta_plus a_plus M_plus mu_plus] =

[V_minus theta_minus a_minus M_minus

mu_minus] = Minus_Var1 (u_minus,v_minus,gamma, R, T0) ;

[lambda_plus Q_plus R_plus S_plus] =

PlusCoeff (theta_plus,mu_plus, u_plus, v_plus, a_plus, r_plus, delta) ;

[lambda_minus Q_minus R_minus S_minus] =

MinusCoeff (theta_minus,mu_minus,u_minus,v_minus,a_minus,r_minus,delta);

$z \_n e w(j, i)=(r(j t o p, i-1)-$

lambda_minus*z (jtop, i-1) -r (jbot, i-1) +lambda_plus*z (jbot, i-

1)) / ( lambda_plus-lambda_minus) ;

$r \_$new $(j, i)=r(j$ bot,$i-1)-$

lambda_plus*z (jbot,i-1)+lambda_plus*z_new $(j, i)$;

1)) $+Q \_p l u s * u(j$ bot, $i-1)+R \_p l u s * v(j b o t, i-1)$;

T_plus $=$ S_plus* $\left(z \_n e w(j, i)-z(j b o t, i-\right.$

T_minus $=S \_m i n u s *\left(z \_n e w(j, i)-z(j t o p, i-\right.$

1)) +Q_minus*u(jtop, i-1)+R_minus*v(jtop,i-1);

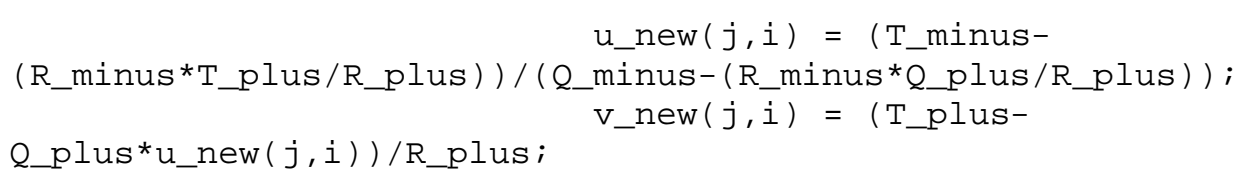

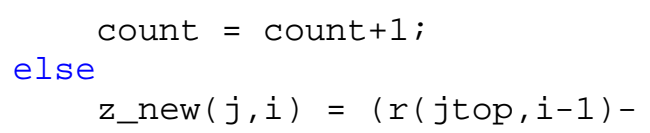

lambda_minus*z (jtop, i-1) -r (jbot, i-1) +lambda_plus*z (jbot, i-

1)) / (lambda_plus-lambda_minus) ;

$r \_$new $(j, i)=r(j$ bot,$i-1)-$

lambda_plus*z (jbot, i-1) +lambda_plus*z_new $(j, i)$;

1)) $+Q \_p l u s * u(j$ bot, $i-1)+R \_p l u s * v(j b o t, i-1)$;

T_plus $=$ S_plus* $\left(z \_n e w(j, i)-z(j b o t, i-\right.$

T_minus $=S \_m i n u s *\left(z \_n e w(j, i)-z(j t o p, i-\right.$

1)) $+Q \_$minus*u (jtop, $\left.i-1\right)+R \_m i n u s * V(j t o p, \bar{i}-1)$; 


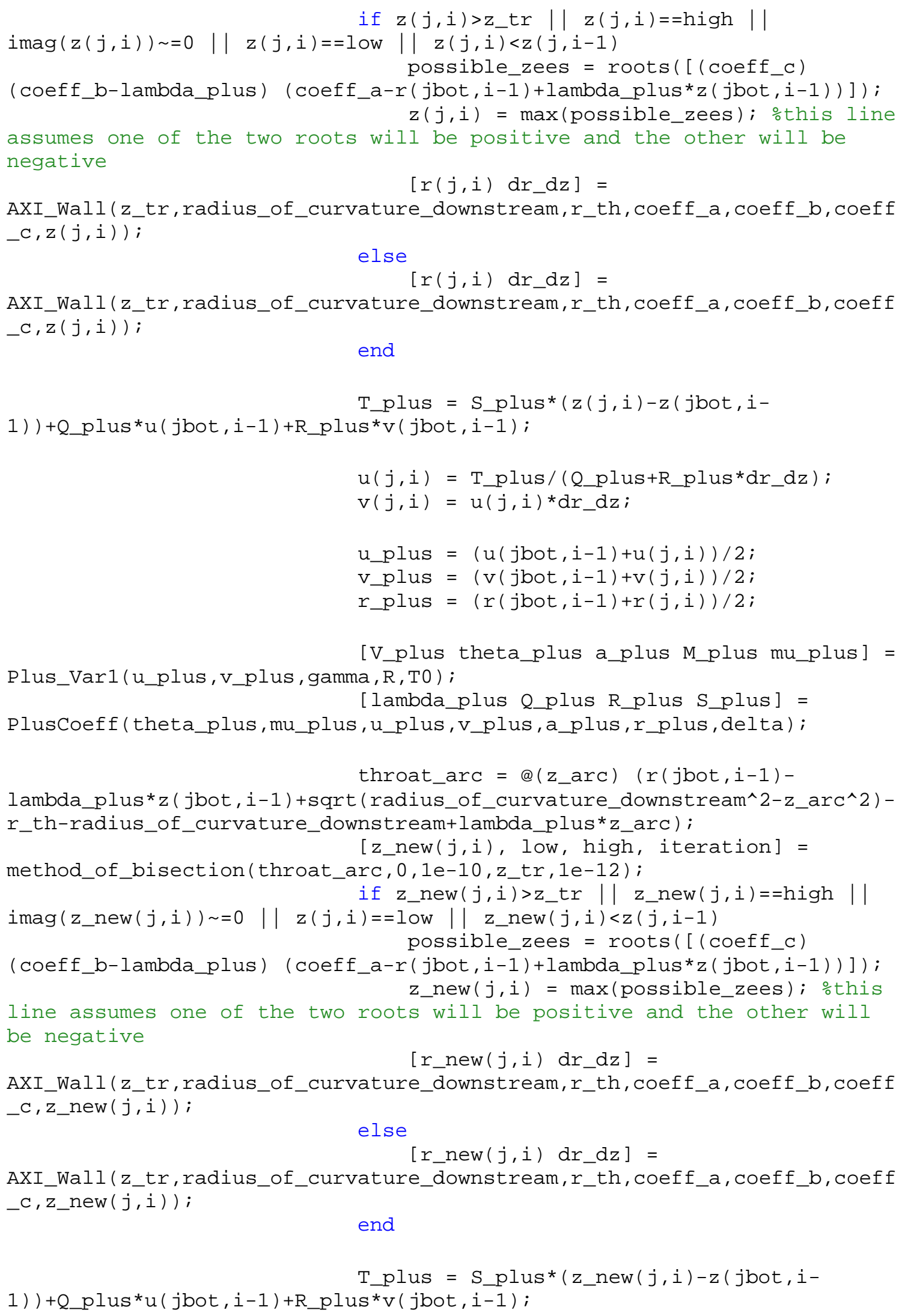




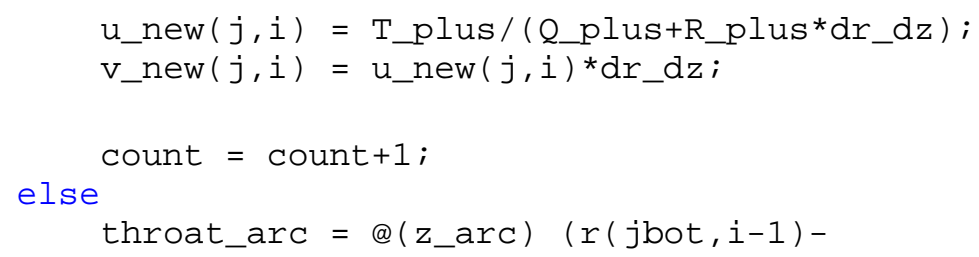

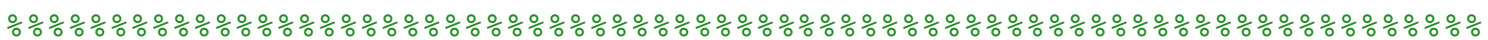

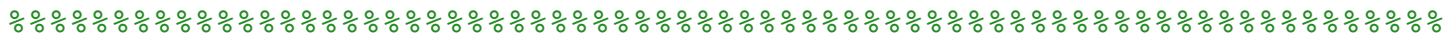

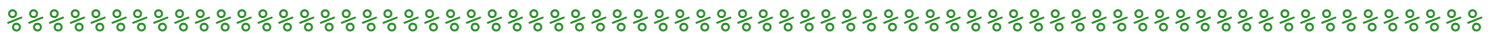

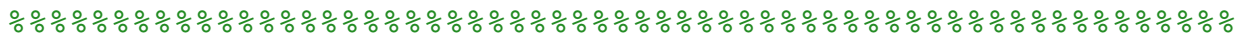

CASE \#3: INTERIOR POINTS (fall neither on the

centerline or the nozzle

wall)

otherwise

count $=0$; 


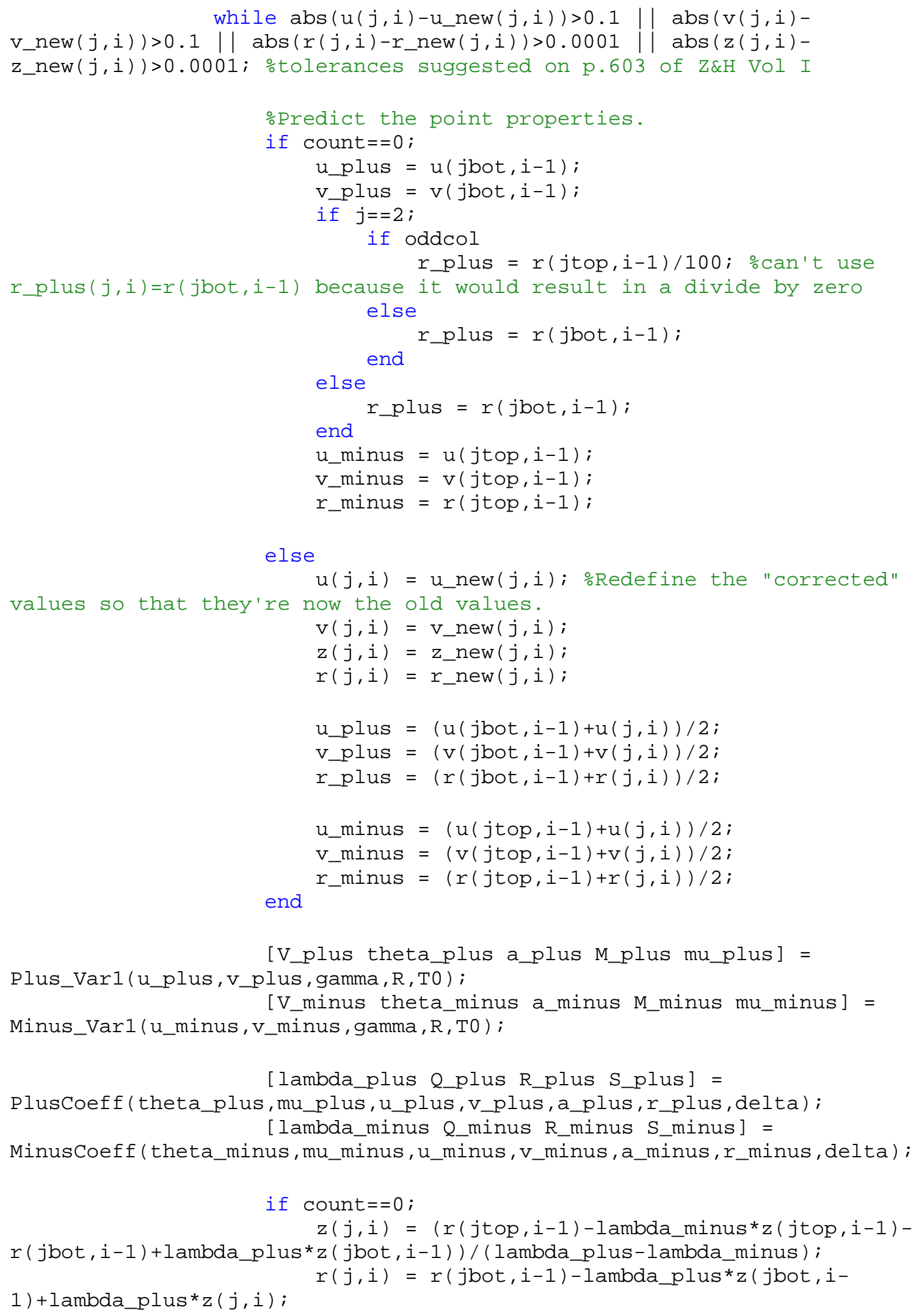




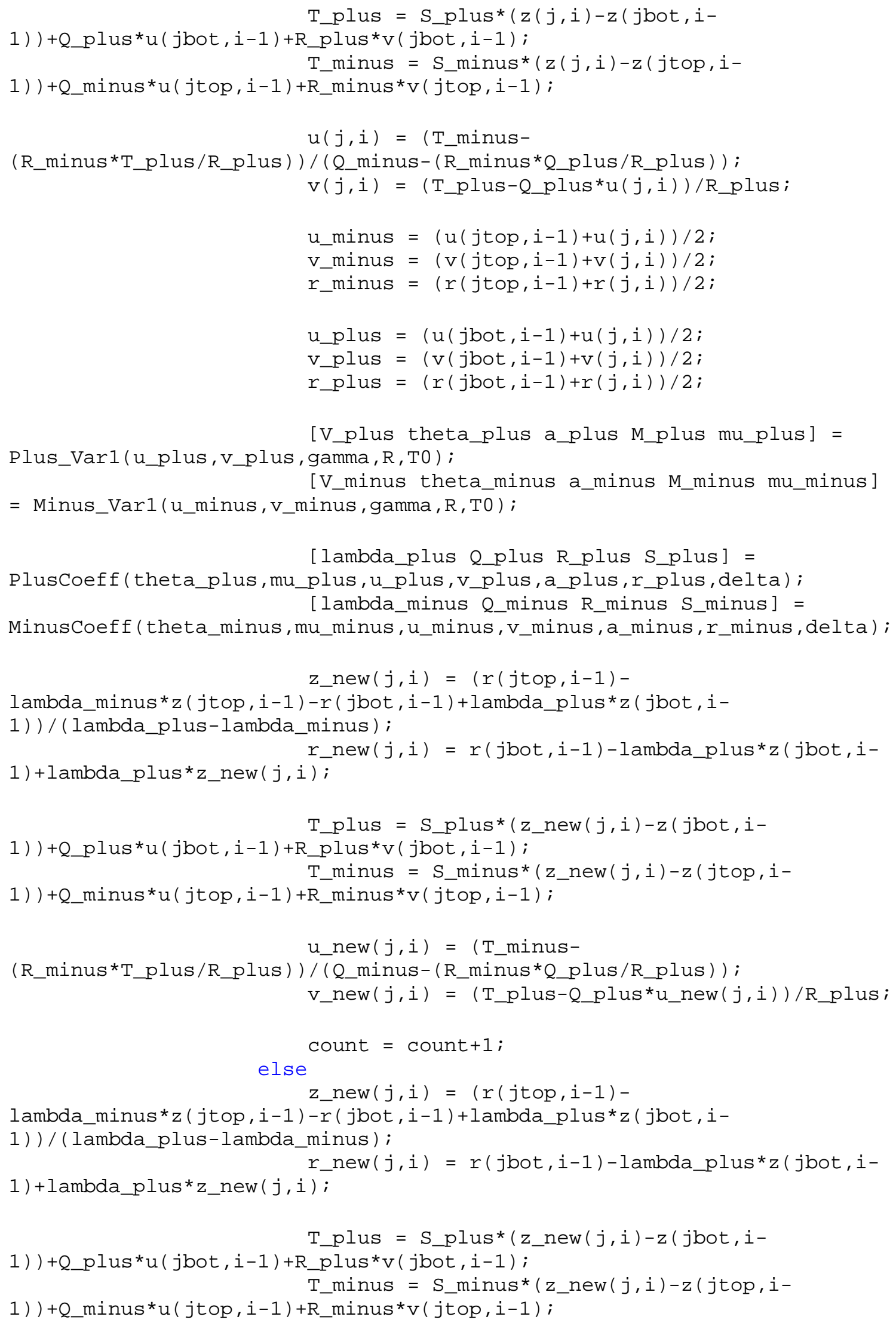




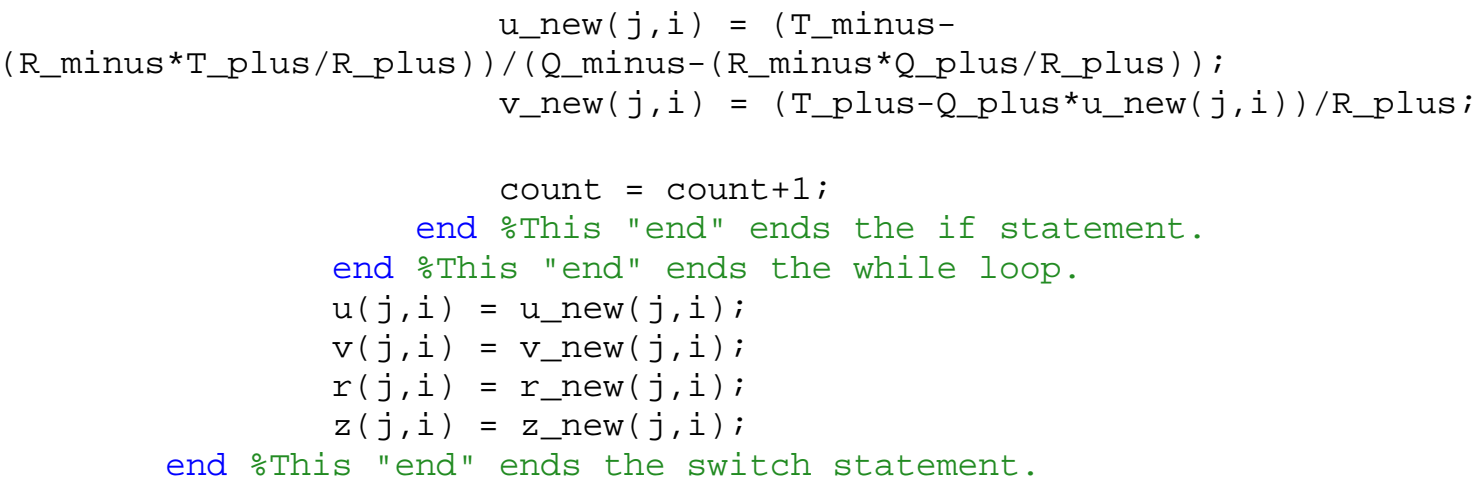

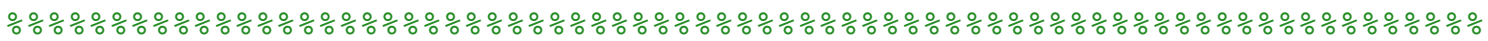

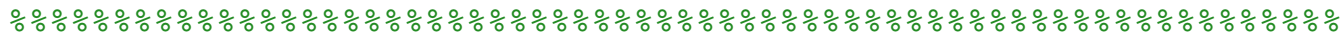

ㅇ Check to see if you're done.

$$
\text { if }(z(\operatorname{JMAX}, i)>=\text { zduct }) \quad|(i>=\operatorname{IMAX}) \quad| \mid \text { (Free }==1 \text { \&\& }
$$

$((\operatorname{Rp}(e n d)<\max (\mathrm{Rp})-\max (\mathrm{Rp}) / 75)|| \max (\mathrm{Rp})>=$ rduct+tand (thetaduct)* $(\max (\mathrm{Zp})-\mathrm{zlen})))$; othis will make sure you're at the end of the nozzle. may still be in the duct

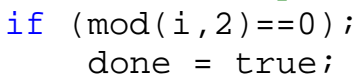


function [Ms Ps Zs mdotstar Zd Rd Pd Md At] =

SSFLOW2 ( Zp, Rp, Zl, Rl, rduct, thetaduct, gamma, pa, Ta, R, rhoa, intfigs, tb)

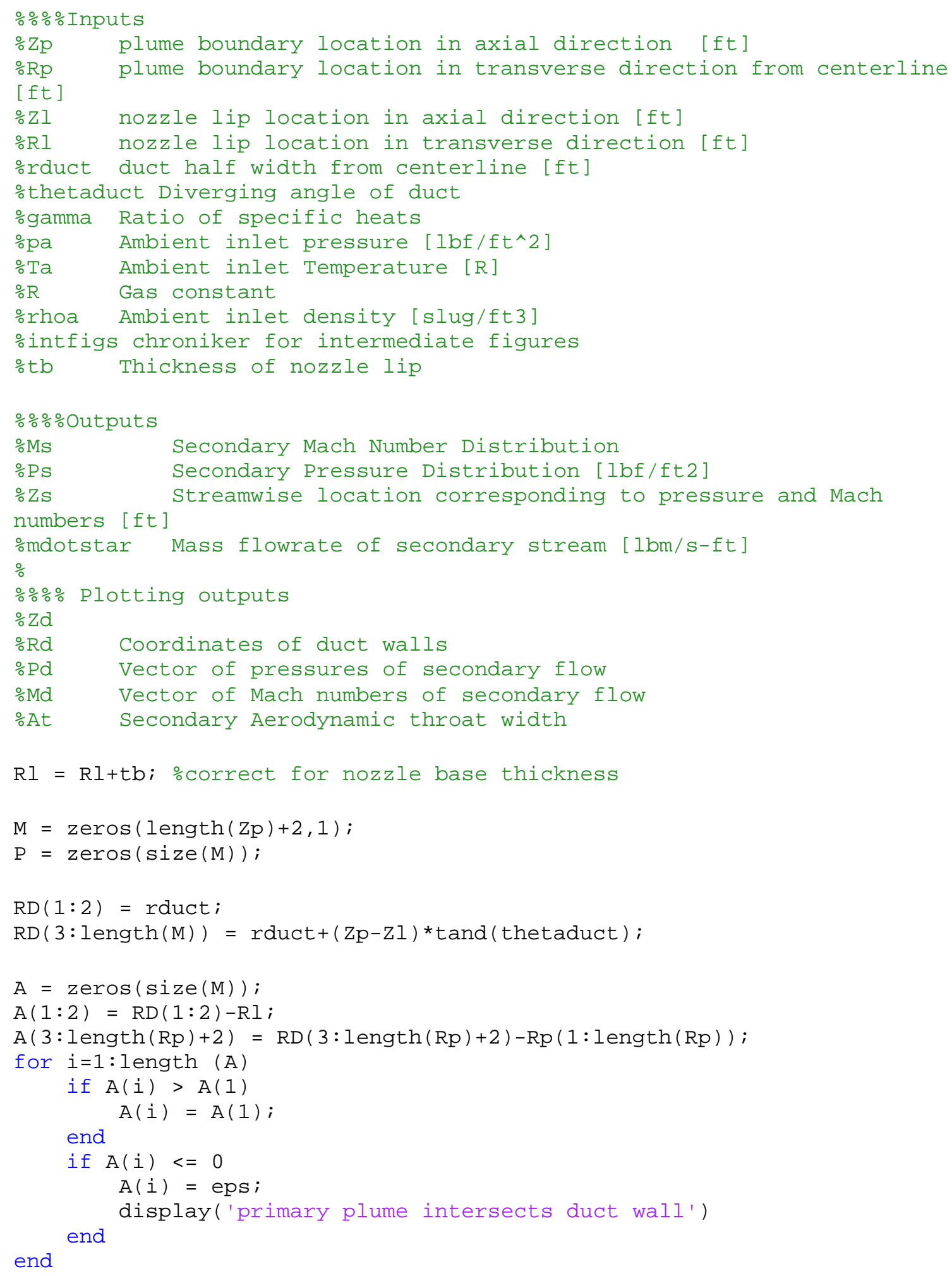




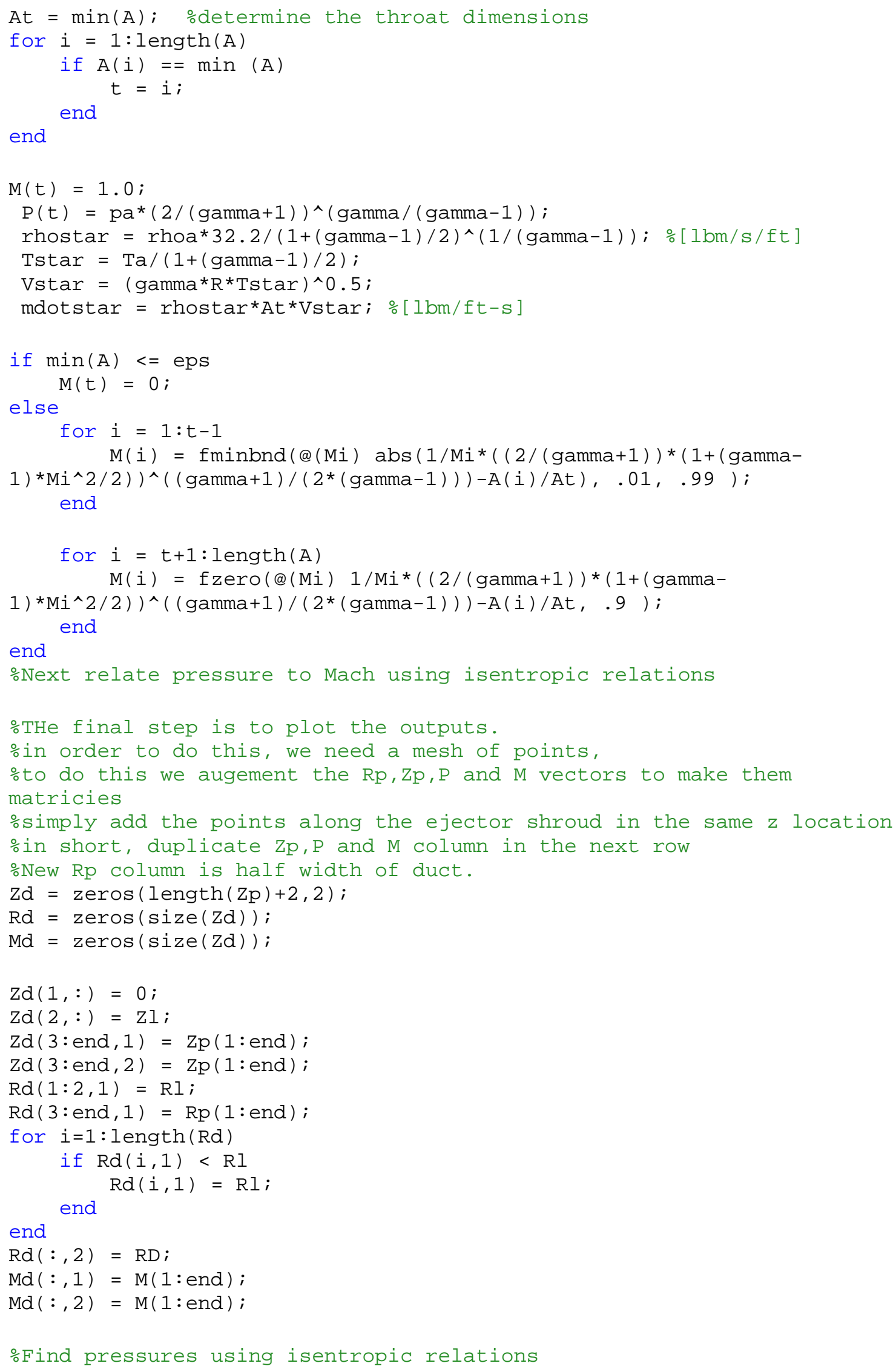




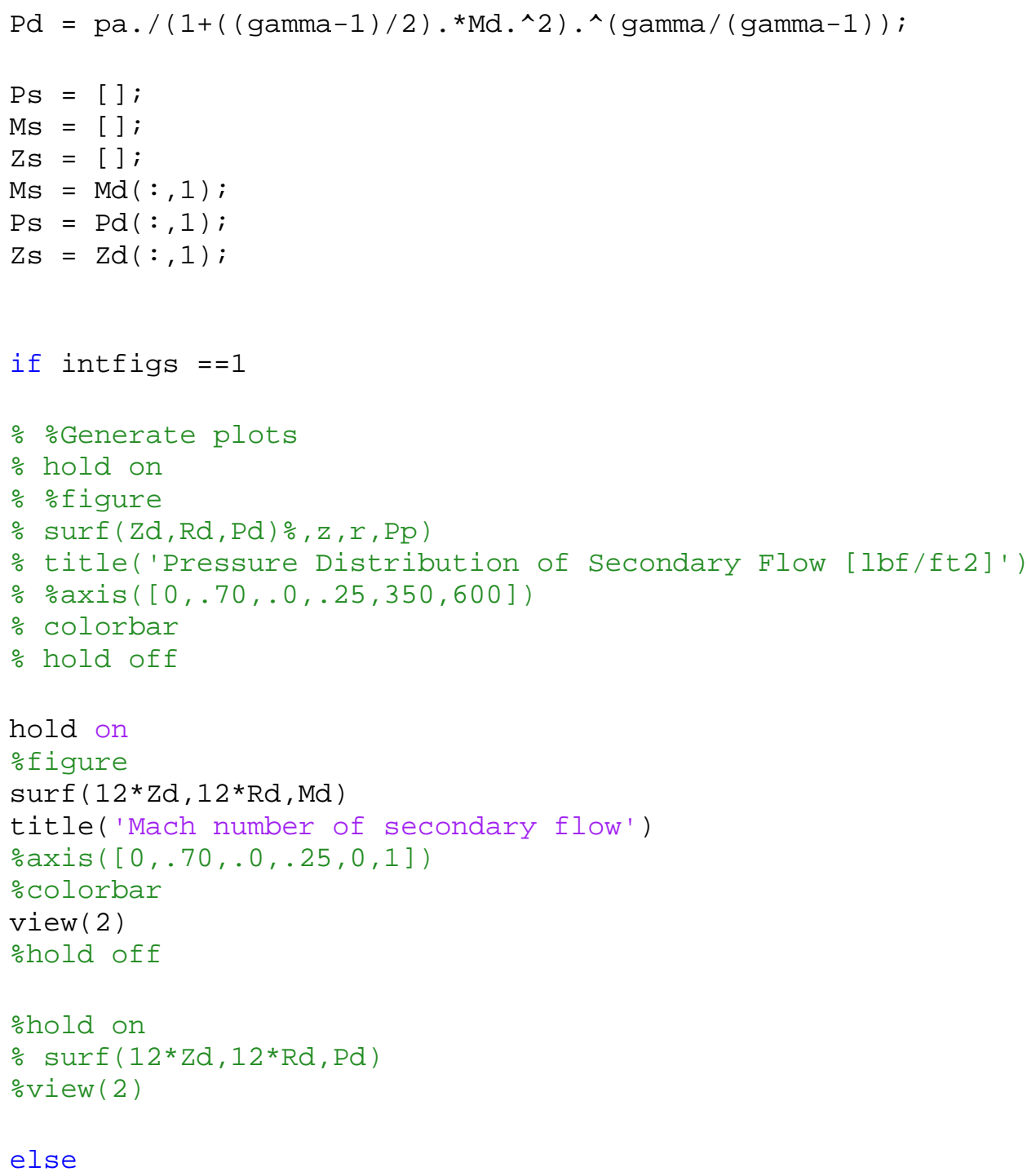


$\div$ r_star $=$ throat radius (ft)

\% $\bar{T} 0$ chamber stagnation temperature (deg $\mathrm{R}$ )

JMAX $=$ number of points in the initial column

T_star $=(2 /(1+$ gamma $)) * \mathrm{~T} 0 ;$ \%throat temperature (deg R)

a_star $=$ sqrt (gamma*gas_constant*T_star); \%throat speed of sound (ft/s)

$\mathrm{R}=$ curvature_upstream/r_star; $\%$ EXPANSION PARAMETER

$y=$ linspace $(0, r$ _star, JMAX) '; \%this is the span of transverse points

from the axis to the throat wall

$r=y \cdot / r \_s t a r ;$ othis is y non-dimensionalized by the value of the

throat radius

$z=0.25-0.25 .{ }^{*} r .{ }^{\wedge} 2 ;$ othis is the axial non-dimensional value that

corresponds to $r$

$\mathrm{x}=($ r_star.*z)/sqrt $((2 * \mathrm{R}) /($ gamma+1) $)$; $\%$ this is the span of axial

points corresponding to $y$

$\mathrm{u} 1=0.5 . \star(r . \wedge 2)-0.25+\mathrm{z} ;$

$\frac{\circ}{0} 1=0.25 \cdot *(r \cdot \wedge 3)-(0.25 \cdot * r)+(r \cdot * z) ;$

$\mathrm{v} 1=0 ;$ Assume $\mathrm{v} 1$ contributes negligibly

$\mathrm{u} 2=\left(((2 *\right.$ gamma +9$\left.) / 24) \cdot{ }^{\star} \mathrm{r} \cdot{ }^{\wedge} 4\right)-$

$\left(\left(\left(4{ }^{*}\right.\right.\right.$ gamma +15$\left.\left.) / 24\right) \cdot{ }^{\star} r .{ }^{\wedge} 2\right)+((10 * g a m m a+57) / 288)+z \cdot{ }^{*}(r . \wedge 2-(5 / 8))-$

$\left(((2 \star\right.$ gamma -3$\left.) / 6) \cdot{ }^{*} \cdot^{\wedge} 2\right) ; \% \mathrm{Z} \& \mathrm{H}$ Vol II Eq. 15.102

$\mathrm{v} 2=\left(((\right.$ gamma +3$\left.) / 9) \cdot{ }^{\star} r \cdot \wedge 5\right)-$

$\left(((20 * g a m m a+63) / 96) \cdot{ }^{\star} r \cdot{ }^{\wedge} 3\right)+\left(((28 * g a m m a+93) / 288) \cdot{ }^{\star} r\right)+z \cdot{ }^{*}((((2 * g a m m a+9) / 6$

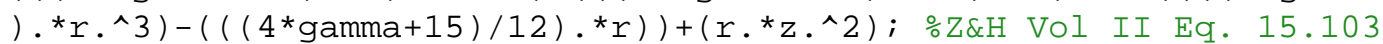

u $3=\left(((556 *\right.$ gamma^2+1737*gamma +3069$\left.) / 10368) \cdot{ }^{\star}{ }^{\wedge}{ }^{\wedge} 6\right)-$

$\left(((388 * g a m m a \wedge 2+1161 * g a m m a+1881) / 2304) \cdot{ }^{\star} r . \wedge 4\right)+(((304 * g a m m a \wedge 2+831 * g a m m a+1$ $\left.242) / 1728) \cdot{ }^{*} r \cdot{ }^{\wedge} 2\right)-$

( (2708*gamma^2+7839*gamma+14211)/82944) +z * ( ( ( (52*gamma^2+51*gamma +327$)$

/384).*r.^4)-

$\left(((52 *\right.$ gamma^2+75*gamma +279$) / 192) \cdot{ }^{\star}$ r.^2) $+(((92 * g a m m a \wedge 2+180 * g a m m a+639) / 11$ $52)))+z \cdot{ }^{\wedge} 2 \cdot *\left(\left(((-7 * g a m m a-3) / 8) \cdot{ }^{\star} r \cdot{ }^{\wedge} 2\right)+((13 * g a m m a-27) / 48)\right)+(((4 * g a m m a \wedge 2-$ $57 *$ gamma +27$) / 144) \cdot * z \cdot \wedge 3) ; \% Z \& H$ Vol II Eq. 15.104

$\mathrm{u} 3=\left(\left(\left(556{ }^{\star} \mathrm{gamma} \wedge 2+1737{ }^{\star} \mathrm{gamma}+3069\right) / 10368\right) \cdot{ }^{\star}{ }^{\wedge} \cdot{ }^{\wedge} 6\right)-$

$\left(((388 * g a m m a \wedge 2+1161 * g a m m a+1881) / 2304) \cdot{ }^{\star} r .^{\wedge} 4\right)+\left(\left(\left(304{ }^{\star} g a m m a \wedge 2+831 * g a m m a+1\right.\right.\right.$ $\left.242) / 1728) \cdot{ }^{*} r \cdot \wedge 2\right) \ldots$

$-$

$((2708 *$ gamma^2+7839*gamma +14211$) / 82944)+z . \star((((52 * g a m m a \wedge 2+51 * g a m m a+327)$

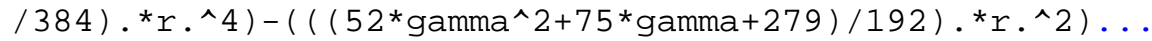

$+(((200 *$ gamma $2+72 * g a m m a+639) / 1152)))+z \cdot{ }^{\wedge} 2 * *((((-7 \star g a m m a-$

$\left.\left.3) / 8) \cdot{ }^{\star} r \cdot{ }^{\wedge} 2\right)+((13 * g a m m a-27) / 48)\right)+\left(((4 * g a m m a \wedge 2-57 * g a m m a+27) / 144) \cdot{ }^{\star} z \cdot{ }^{\wedge} 3\right)$;

$\div \mathrm{Hall} \mathrm{Eq} \cdot 81$

$\mathrm{v} 3=\left(\left(\left(6836{ }^{\star} \mathrm{gamma}{ }^{\wedge} 2+23031{ }^{\star} \mathrm{gamma}+30627\right) / 82944\right) .{ }^{\star} \mathrm{r} \cdot{ }^{\wedge} 7\right)-$

( ( (3380*gamma^2+11391*gamma+15291)/13824) .*r.^5)+( ( (3424*gamma^2+11271* gamma+15228)/13824) .*r.^3)-

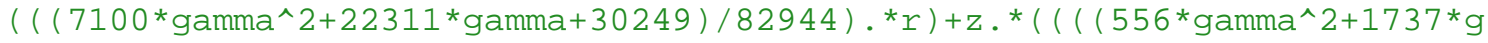
amma+3069) /1728) $\left.\cdot{ }^{\star} r \cdot{ }^{\wedge} 5\right)-$

$(((388 *$ gamma^2+1161*gamma+1881)/576) .*r.^3)+(( (304*gamma^2+831*gamma+12 $\left.\left.42) / 864) .{ }^{\star} r\right)\right)+z \cdot{ }^{\wedge} 2 . *\left(\left(((52 * g a m m a \wedge 2+51 * g a m m a+327) / 192) \cdot{ }^{\star} r . \wedge 3\right)-\right.$

$\left.\left(\left(\left(52{ }^{\star} g a m m a \wedge 2+75 * g a m m a+279\right) / 192\right) \cdot{ }^{\star} r\right)\right)-z \cdot{ }^{*} 3 . *\left(((7 * g a m m a-3) / 12) \cdot{ }^{*} r\right) ; \% Z \& H$

Vol II Eq. 15.105 


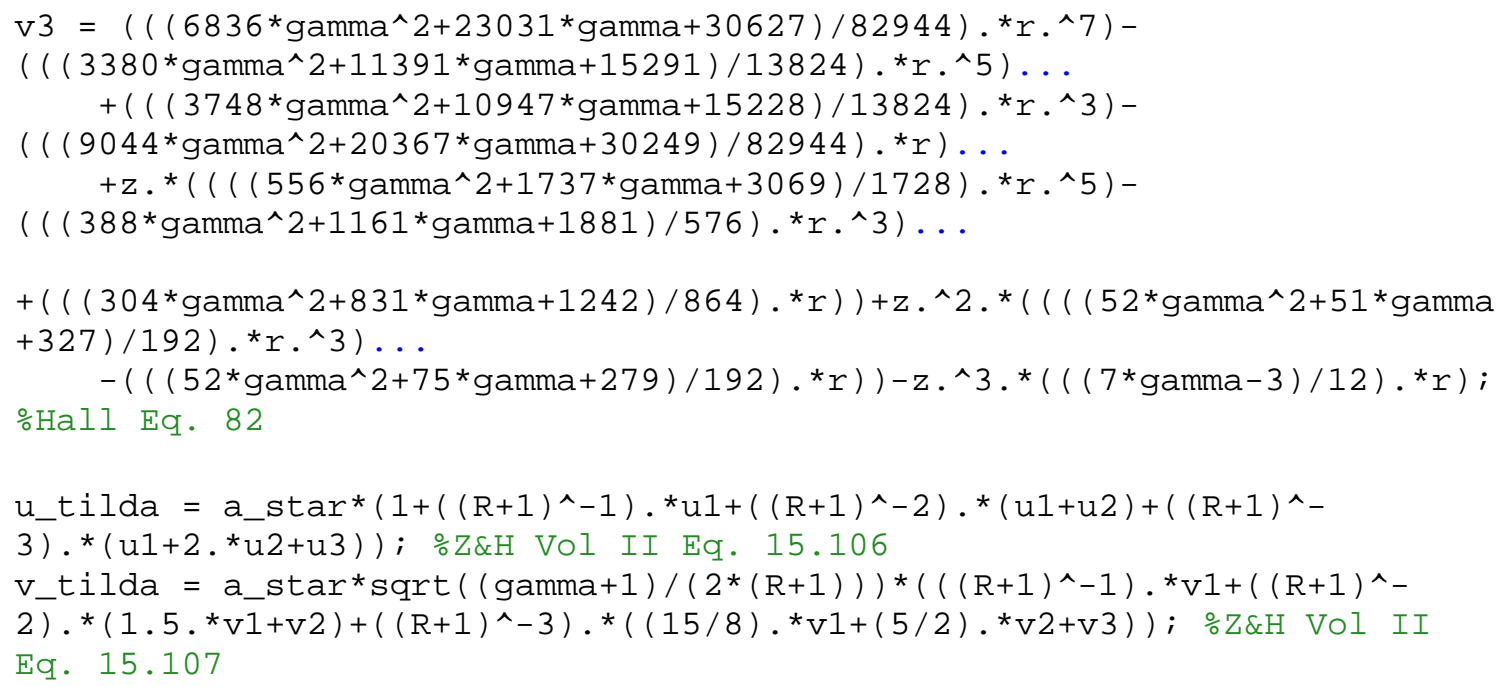

function [V_minus theta_minus a_minus M_minus mu_minus] =

Minus_Var1 (u_minus, v_minus,gamma, R, T0)

V_minus $=\operatorname{sqrt}\left(u \_m i n u s^{\wedge} 2+v \_\right.$minus^2);

theta_minus $=\operatorname{atan}\left(v \_m i n u s / u \_m i n u s\right)$;

a_minus $=$ speed_of_sound (gamma, R, T0,V_minus) ;

M_minus = V_minus/a_minus;

mu_minus = mach_angle (M_minus);

end

function [lambda_minus Q_minus R_minus S_minus] =

MinusCoeff (theta_minus,mu_minus, u_minus,v_minus,a_minus,r_minus, delta)

lambda_minus $=$ tan (theta_minus-mu_minus);

Q_minus $=$ u_minus^2-a_minus^2;

R_minus $=2{ }^{*}$ u_minus*v_minus_Q_minus*lambda_minus;

S_minus $=$ delta*a_minus^ $2{ }^{*}$ v_minus/r_minus;

end

function [lambda_plus Q_plus R_plus S_plus] =

PlusCoeff (theta_plus,mu_plus, u_plus, v_plus, a_plus, r_plus, delta)

lambda_plus = tan (theta_plus+mu_plus);

Q_plus $=$ u_plus^2-a_plus^2;

R_plus $=2 * u \_p l u s * v \_p l u s-Q \_p l u s * 1$ ambda_plus ;

S_plus $=$ delta*a_plus^ ${ }^{\star}{ }^{*}$ __plus/r_plus;

end

function [V_plus theta_plus a_plus M_plus mu_plus] =

Plus_Var1 (u_plus, v_plus, gamma, R, T0)

V_plus $=$ sqrt $\left(u \_p l u s^{\wedge} 2+v_{\text {_plus^}} 2\right)$; 
theta_plus = atan (v_plus/u_plus);

a_plus $=$ speed_of_sound (gamma, R, T0,V_plus) ;

M_plus $=$ V_plus/a_plus;

mu_plus = mach_angle (M_plus);

end

This portion correlates to the Z\&H example. R may be different units. omu_plus is also named alpha_plus

function $[M$ P R T C ] = THERMO (Q, GC, CP, gamma, RG, PO, T0)

oThis sub code has unit issues

$\mathrm{CP}=$ gamma ${ }^{\mathrm{RG}} /($ gamma-1); $\% \mathrm{CP}$ is $\mathrm{Cp}$ not speed-of-sound_pluss

$\mathrm{GC}=1$; othis solves a unit issue (thermo requires $\mathrm{RG}$ in ftlbf/lbmR. input as ftlbf/slugR=ft^2/Rs^2)

$\mathrm{T}=\mathrm{T} 0-\mathrm{Q}^{\wedge} 2 /(2 * \mathrm{GC} * \mathrm{CP}) ;$

$\mathrm{C}=\left(\text { gamma* } \mathrm{GC}^{\star} \mathrm{RG} \mathrm{T}^{\star}\right)^{\wedge} .5$; \%speed of sound \%this value is too large by a

magnitude

$\mathrm{M}=\mathrm{Q} / \mathrm{C}$;

$\mathrm{P}=\mathrm{PO} *(\mathrm{~T} / \mathrm{TO})^{\wedge}($ gamma/(gamma-1)) $;$ \% was $144 \mathrm{Cf}$,

$\mathrm{R}=\mathrm{P} /(\mathrm{RG} * \mathrm{~T}) * 32.2$; odensity. 32.2 added to change slugs to l.bm

function acoustic_speed = speed_of_sound (gamma, R, T0,V)

This function calculates the local speed of sound.

ogamma is the ratio of specific heats.

$\circ \mathrm{R}$ is the gas constant.

TO is the stagnation temperature.

$\circ \mathrm{V}$ is the local velocity.

acoustic_speed $=\operatorname{sqrt}\left(\right.$ gamma ${ }^{\star} \mathrm{R}^{\star} \mathrm{T} 0-(($ gamma -1$\left.) / 2){ }^{\star} \mathrm{V}^{\wedge} 2\right)$;

end 